\title{
Phosphorhaushalt und planktische Primärproduktion im Vierwaldstättersee (Horwer Bucht)
}

\author{
VON RENÉ GÄCHTER
}

Eidg. Anstalt für Wasserversorgung, Abwasserreinigung und Gewässerschutz an der Eidg. Techn. Hochschule Zürich

Direktion: Prof. Dr. O. Jaag

\section{INHALTSVERZEICHNIS}

1. Einleitung und Problemstellung . . . . . . . . . . . . . . . . . 2

2. Methodik . . . . . . . . . . . . . . . . . . . . . . . . 5

2.1 Physikalische Bestimmungsmethoden . . . . . . . . . . . . . . . . . 6

2.2 Chemische Analysenmethoden . . . . . . . . . . . . . . . . . . . . . . 6

2.3 Biologische Untersuchungsmethoden . . . . . . . . . . . . . . . . . . . . . . 7

3. Seeuntersuchungen ......................... 7

3.1 Lage und Orographie des Gewässers . . . . . . . . . . . . . . . . . . . . . . 7

3.2 Physikalische Untersuchungen . . . . . . . . . . . . . . . . . . . . . . 8

3.2.1 Temperatur . . . . . . . . . . . . . . . . . . . . . . . 8

3.2 Lichtmessungen . . . . . . . . . . . . . . . . . . . . . . . . . . . . 8

3.3 Chemische Untersuchungen . . . . . . . . . . . . . . . . . . . . . . . 10

3.3.1 Sauerstoff . . . . . . . . . . . . . . . . . . . . . . 10

3.3.2 Stickstoff . . . . . . . . . . . . . . . . . . . . . . . 12

3.3.3 Phosphat $\left(\mathrm{PO}_{4}\right)$. . . . . . . . . . . . . . . . . . . . . . . . . 13

3.3.4 Gelöste organische Phosphorverbindungen (GOP) . . . . . . . . . . . . . . . 16

3.3.5 Geformter oder partikulärer Phosphor (PP) . . . . . . . . . . . . . . . . . . 18

3.3.6 Gesamtphosphor . . . . . . . . . . . . . . . . . . . . . . . . . . . . . 19

3.3.7 Phosphorzufuhr zur trophogenen Schicht . . . . . . . . . . . . . . . 24

3.4 Biologische Untersuchungen. . . . . . . . . . . . . . . . . . . . . . . . . . 25

3.4.1 Qualitative und quantitative Planktonanalysen . . . . . . . . . . . . . . 25

3.4.2 Primärproduktion und Phosphor . . . . . . . . . . . . . . . . . . . . . . . 28

3.4.3 Primärproduktion und Licht . . . . . . . . . . . . . . . . 30

4. Versuche . . . . . . . . . . . . . . . . . . . . . . . . . 32

4.1 Versuch vom 27.7.66 bis 14.8.66 . . . . . . . . . . . . . . . . . . 35

4.2 Versuch vom 11. 10.66 bis 20.10 .66 . . . . . . . . . . . . . . . . . . . . . 39

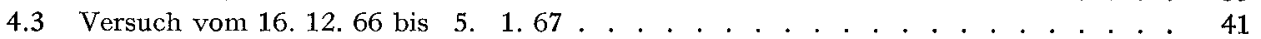

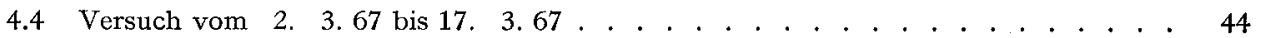

4.5 Versuch vom 28.4 .67 bis 16.5 .67 . . . . . . . . . . . . . . . . . . 46

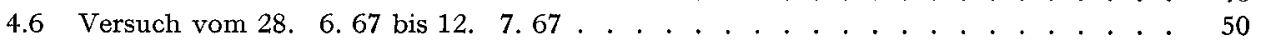


5. Diskussion . . . . . . . . . . . . . . . . . . . . . . . . . . 54

5.1 Phosphataufnahme . . . . . . . . . . . . . . . . . . . . . . . . 54

5.2 Sedimentationsverluste . . . . . . . . . . . . . . . . . . . . . . . . 55

5.3 Einfluss der künstlich erhöhten Gesamtphosphorkonzentration auf die Entwicklung einzelner Phytoplankter . . . . . . . . . . . . . . . . . . . . . . . . . .

5.4 Einfluss der künstlich erhöhten Gesamtphosphorkonzentration auf die planktische Primärproduktion im Verlaufe eines Jahres . . . . . . . . . . . . . . . . . . . . . . 60

6. Schlussfolgerungen . . . . . . . . . . . . . . . . . . . . . . . . . . . 62

7. Zusammenfassung .. . . . . . . . . . . . . . . . . . . . . . 62 Summary . . . . . . . . . . . . . . . . . . . . . . . . . 64

\section{Einleitung und Problemstellung}

Wie aufgrund von Untersuchungen an Sedimentbohrkernen gezeigt werden kann, gehörten die grösseren und tieferen Seen der Nordschweiz bis vor wenigen Jahrzehnten dem oligotrophen Typus an $[1,2]$. Ihr Wasser war nährstoffarm, und der im Wasser gelöste Sauerstoff muss im allgemeinen genügt haben, um die autochthon produzierte und allochthon eingeschwemmte organische Substanz auf aerobem Wege abzubauen. Heute jedoch befindet sich ein grosser Teil dieser Seen im eutrophen Zustand. Ihr Wasser ist nicht mehr blaugrün und klar, sondern meist trübe und weist je nach Jahreszeit mancherlei Vegetationsfärbung auf. Massenentfaltungen von verschiedenen Phytoplanktern im Pelagial und Veralgung und Verkrautung der Litoralzone sind charakteristische Erscheinungen für diesen Seentyp. Der gelöste Sauerstoff reicht nicht mehr aus, um die allochthon und autochthon produzierte organische Substanz vollständig zu mineralisieren. Das Sediment ist nicht mehr hellgrau oder braun, sondern periodisch oder dauernd durch Eisenmonosulfid schwarz gefärbt.

Für diese Eutrophierungserscheinungen wird die vermehrte Nährstoffzufuhr durch Abwasser sowie die Erosion und Auswaschung von landwirtschaftlich intensiv genutztem Kulturland verantwortlich gemacht. Zwar bewirken mechanisch-biologisch gereinigte Abwässer keine unmittelbare Verschmutzung des Vorfluters mehr, enthalten aber beträchtliche Mengen an Nährsalzen gelöst und begiunstigen daher, dank ihrer düngenden Wirkung, das Wachstum von Algen und höhern Wasserpflanzen.

Im natürlichen Gewässer sind die zehn als Kernnährstoffe geltenden Elemente von SACHS und die zum Wachstum nötigen Spurenelemente nicht dem Bedarf des Planktons entsprechend im richtigen Verhältnis gelöst. In den meisten Fällen ist zum Beispiel das Kohlenstoffangebot viel zu gross, während die gelösten Phosphor- und Stickstoffverbindungen als wichtige weitere Nährstoffe nur in unverhältnismässig kleinen Konzentrationen auftreten. Das Liebigsche "Gesetz vom Minimum» besagt, dass derjenige unter den verschiedenen Wirkungsfaktoren, der am meisten im Minimum ist, den gesamten Ertrag bestimme.

MitscherLichs [3] Versuche mit Ackerpflanzen ergaben, dass der Ertrag nicht nur durch den am meisten im Minimum befindlichen Faktor bestimmt werde, sondern dass jeder Wachstumsfaktor eine ganz bestimmte qualitative und quantitative Wirkung auf die Ertragshöhe von Pflanzen habe. Das von ihm formulierte Gesetz lautet: "Mit der Steigerung eines jeden dieser Faktoren steigt der Pflanzenertrag proportional dem Ertrage, welcher an dem unter den gegebenen Bedingungen erzielbaren Höchstertrage fehlt. Dieser Anstieg muss um so schneller erfolgen, je grösser 
der dem einzelnen Wachstumsfaktor typische Wirkungsfaktor ist und je geringere Mengen des betreffenden Faktors der Pflanze bisher zur Verfügung standen." VERDUIN [4] geht mit MIscheRLICH einig und betont, dass das Wirkungsgesetz der begrenzenden Faktoren nicht allzusehr simplifiziert werden darf, wenn es in seiner Bedeutung richtig bleiben soll. Wird daher ein Faktor dann als begrenzend bezeichnet, wenn eine Erhöhung seiner Wirkung (Konzentration oder Intensität) eine höhere Wachstumsrate bewirkt, so können verschiedene Faktoren gleichzeitig begrenzend wirken.

Für den praktischen Seenschutz, dessen Ziel es ist, die trophische Entwicklung der Seen zu stoppen oder zumindest zu verlangsamen, bedeutet das, dass eine weitere Zufuhr jener Stoffe, die das Ausmass der Primärproduktion entscheidend zu steigern vermögen, unterbunden werden muss. Manche Autoren nennen Phosphate, andere Stickstoffverbindungen als Urheber der Eutrophierung, und eine dritte Gruppe, die den Einfluss von Vitaminen und Wuchsstoffen untersucht, zeigt, dass Massenentfaltungen von planktischen Algen mit mineralischen Nährstoffanalysen allein oft nicht erklärt werden können [5].

In schweizerischen Alpenrandseen treten Phosphorverbindungen natürlicherweise nur in sehr niedrigen Konzentrationen auf. Stickstoffyerbindungen werden leichter aus Kulturböden ausgewaschen als Phosphate, Regenwasser kann beträchtliche Mengen Nitrat enthalten, und zudem vermögen einige Blaualgen und Bakterien elementaren Stickstoff zu assimilieren [6-10]. Es wird daher angenommen $[11,12]$, dass in den meisten grossen Alpenrandseen und namentlich im Vierwaldstättersee, wo die epilimnischen Phosphatkonzentrationen oft unter $0,2 \mu \mathrm{g} \mathrm{PO}_{4}-\mathrm{P} / \mathrm{I}$ sinken, in erster Linie Phosphat der begrenzende Faktor der photosynthetischen Primärproduktion sei. Einschränkend muss hier erwähnt werden, dass nach einer Zusammenstellung von Lund [13] keine der im Vierwaldstättersee gefundenen Blaualgen mit Sicherheit elementaren Sickstoff zu assimilieren vermag und dass, wie Versuche von MAckereTH [14] an Asterionella formosa gezeigt haben, der chemisch bestimmte Wert $0 \mu \mathrm{g} \mathrm{P} / 1$ von der Empfindlichkeit der Analysenmethode abhängt und vom physiologischen Nullwert noch weit entfernt sein kann. GESSNER [15] formulierte daher den Satz: "Die Frage, ob ein Stoff im Wasser die Rolle eines Minimumfaktors spielt, mündet also in das Problem ein, welche Kräfte den Zellen zur Verfügung stehen, um Ionen an sich zu reissen."

Ein weiterer Hinweis auf einen möglichen Zusammenhang zwischen Erhöhung der Phosphatkonzentration und dem Anstieg der Phytoplanktondichte ist aus Untersuchungen am Bodensee bekannt $[16,17]$. Es gilt als unbestritten, dass die zunehmende rasche Eutrophierung der Seen auf Abwassereinflüsse und intensiv betriebene Landwirtschaft zurückzuführen sind. Dabei gelangen aber neben den Phosphaten noch viele andere Nähr- und Spurenelemente in den See, die für die erhöhte Algenproduktion verantwortlich gemacht werden könnten. Der Anstieg der Phosphatkonzentration und der wachsende "standing crop» des Phytoplanktons in den letzten Jahrzehnten sind also vorerst nur als zwei parallel verlaufende Erscheinungen zu werten, zwischen welchen kein kausaler Zusammenhang bewiesen ist.

Aufgrund der angeführten Analogieschlüsse kann zwar eine begrenzende Wirkung des Phosphors vermutet werden, sicher erkannt wird sie jedoch nur, wenn eine kontrollierte Erhöhung der Phosphatkonzentration vom Gewässer mit erhöhter 
Produktion beantwortet wird. Das bedeutet, dass es unumgänglich ist, zur Beantwortung dieser Fragen das Experiment zu Hilfe zu nehmen.

Thomas [11] und Bringmann und Kühn [18] und Skulberg [19] beschreiben Methoden zur qualitativen Erkennung von Minimumsstoffen und zur quantitativen Erfassung ihrer Begrenzungswirkung. Dabei wird das zu testende Wasser mit Algen beimpft und der Zuwachs bei verschiedener Nährstoffzugabe nach mehrtägiger Expositionszeit bestimmt. In allen Experimenten, in welchen die Konzentration eines Nährstoffs sehr stark erhöht wird, besteht aber die Gefahr, dass Spurenelemente als Verunreinigung in solchen Konzentrationen eingeschleppt werden, so dass sie das Wachstum zu stimulieren vermögen. Das bedeutet, dass im Extremfall nicht die Wirkung des zugesetzten Nährsalzes, sondern jene seiner Verunreinigungen gemessen würde. Die zugesetzten Nährstoffmengen sollten daher möglichst niedrig gehalten werden. Ferner gilt es zu bedenken, dass die in der angewandten Limnologie im Zusammenhang mit Eutrophierungsproblemen gestellten Fragen durch die spezifische Reaktion eines isolierten Organismus unter künstlichen Bedingungen nur entfernt beantwortet werden. Unter Umständen erhält man aber mit in situ ausgeführten Experimenten, wie sie von Goldman [20], Ryther und Guillard [21], Mentzel und RYTHER [22], GOLDMAN [23] und STRICKLAND [24] beschrieben wurden, Resultate, die besser auf natürliche Verhältnisse extrapoliert werden können. Obwohl auch in solchen Experimenten neben den gewollten stets auch unerwünschte, schlecht oder überhaupt nicht kontrollierbare Veränderungen in das zu untersuchende System eingeführt werden, scheinen sie uns geeignet, als Ergänzung zu Freilanduntersuchungen, zur Lösung der Frage über den Zusammenhang von Primärproduktion und Phosphorhaushalt beitragen zu können. Angeregt durch STRICKLAND [25] wurden daher «Nährstofftestzellen" konstruiert, die es erlauben, den Einfluss von zugeführten Nährstoffen auf die Entwicklung von natürlichen Planktonpopulationen in etwa $1400 \mathrm{I}$ Kulturvolumen in situ zu verfolgen.

Ziel dieser Arbeit ist es, die jahreszeitlich bedingten Veränderungen des limnischen Phosphorhaushalts so weit als möglich zu erfassen, durch In-situ-Experimente mit Hilfe der $\mathrm{C}^{\mathbf{1 4}}$-Methode zu prüfen, ob durch Phosphatdüngung eine erhöhte Kohlenstoffassimilation bewirkt werde, und abzuklären, ob und wie weit sich durch diese Düngung qualitative Veränderungen im Phytoplankton ergeben.

Zudem soll aufgrund von monatlich durchgeführten chemischen und biologischen Erhebungen versucht werden, den Trophiegrad des untersuchten Seeteils festzustellen; dies erstens, um es dem Leser zu ermöglichen, sich vom Gewässer ein möglichst genaues Bild zu machen, und zweitens, um für die limnologische Beurteilung der Auswirkungen projektierter Sanierungsmassnahmen eine Basis zu schaffen, denn die Gemeinde Horw (10000 Einwohner) plant in naher Zukunft, die Abwässer gänzlich vom See fernzuhalten.

Diese Arbeit wurde im Hydrobiologischen Laboratorium der ETH in Kastanienbaum ausgeführt. Herrn Prof. Dr. O. Jaag, Direktor der Eidgenössischen Anstalt für Wasserversorgung, Abwasserreinigung und Gewässerschutz (EAWAG), der das Laboratorium untersteht, möchte ich für die Bereitstellung von Arbeitsräumen und Material und die grosszügige finanzielle Unterstützung dieser Arbeit herzlich danken. Danken möchte ich auch Herrn Dr. H. Ambühl, dem Leiter der Limnologischen Abteilung der EAWAG und des Laboratoriums Kastanienbaum für sein Interesse, 
das er dieser Arbeit entgegenbrachte, für die vielen Hinweise und die Kritische Sichtung des Manuskriptes. Zu Dank verpflichtet bin ich auch Fräulein Dr. Marianne Pavoni für die Einführung in den Formenkreis des Phytoplanktons, Frau M. M. Bezzegh-Galántai und ihren Mitarbeitern für die Messung der vielen C14-Proben und Herrn E. Schwager und allen andern Mitarbeitern der Versuchsanlage Tüffenwies für die grosse Hilfsbereitschaft und die prompte Ausführung der Nährstofftestzellen und den Bau des Rotationslichtthermostaten. Danken möchte ich schliesslich den Herren M. Egger, H. Bachmann, B. Ribi und M. Schmid für manche Mithilfe beim Exponieren der Nährstofftestzellen sowie Herrn dipl. sc. nat. U. Zimmermann für die Mithilfe bei sämtlichen Probenahmen, für die Überlassung seiner Planktonzählresultate und die vielen Hinweise und Ideen, die sich aus ungezählten, ausgedehnten Diskussionen mit ihm ergaben.

\section{Methodik}

In den Jahren 1965-1967 wurden an dem in Abb. 1 bezeichneten Punkt vom verankerten Schiff aus monatlich Proben erhoben. Die genaue Stelle wurde jeweils mit Hilfe von Fixpunkten am Land mit einem Pentaprisma eingemessen. Das Wasser wurde mit einem Friedinger Wasserschöpfer von 51 Volumen aus folgenden Tiefen entnommen : $0,0 \mathrm{~m},(1,0 \mathrm{~m}), 2,5 \mathrm{~m}, 5,0 \mathrm{~m}, 7,5 \mathrm{~m}, 10,0 \mathrm{~m}, 12,5 \mathrm{~m}, 15,0 \mathrm{~m},(17,5 \mathrm{~m}), 20,0 \mathrm{~m}$, $30,0 \mathrm{~m}, 50,0 \mathrm{~m}, 71,0 \mathrm{~m}$. Die Wassertiefe beträgt rund $72 \mathrm{~m}$. Die Sauerstoffproben (rund $300 \mathrm{ml}$ ) wurden nach WinkLER [26], das Phytoplankton mit Lugolscher Lösung (Jod-Jodkali) nach UTERMöHL [27] an Ort und Stelle sofort fixiert.

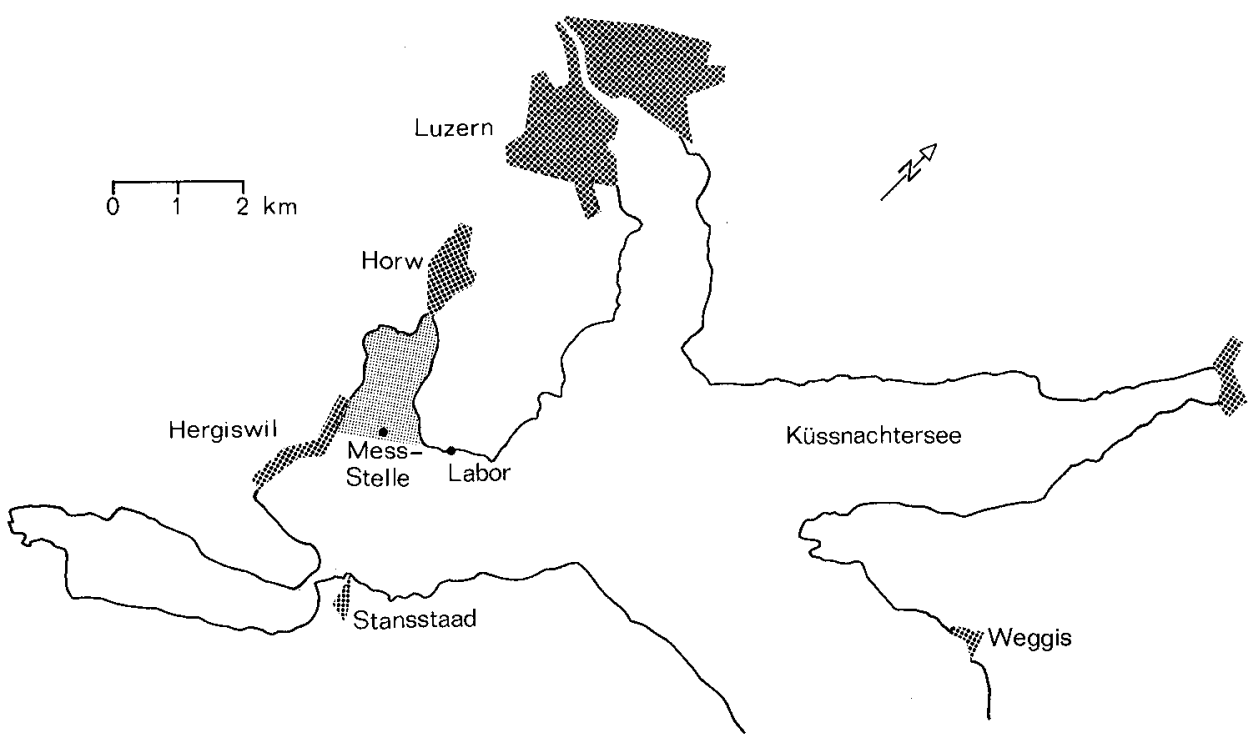

Abb. 1. Tage der Meßstelle. 


\subsection{Physikalische Bestimmungsmethoden}

1. Temperatur: Die Temperaturen wurden mit dem «Oxytester» [28] elektrometrisch bestimmt.

2. Optik: Die vertikalen Extinktionskoeffizienten von verschiedenen Wellenlängen wurden mit einem Selenphotoelement gemessen. Dabei wurden folgende Filterkombinationen vorgeschaltet:

\begin{tabular}{llll}
\hline Filter: & BG12 & VG9 & RG2 \\
\hline Spektralbereich $[\mathrm{nm}]:$ & $320-520$ & $440-660$ & $600-740$ \\
\hline
\end{tabular}

\subsection{Chemische Analysenmethoden} fasst:

Neben Sauerstoff und pH-Wert wurden folgende Komponenten quantitativ er-

Aus dem Rohwasser: Gesamtphosphorkonzentration und Säurebindungsvermögen (SBV). Aus dem Filtrat (Filtration durch gewaschene Filter MF50 der Membranfiltergesellschaft Göttingen): Gesamtphosphor-, Phosphat- und Nitrationenkonzentration.

Es wurden dabei folgende Methoden angewandt:

Sauerstoff: Zur Ermittlung des Sauerstoffgehalts wurde etwa ein Drittel des Wassers über dem sedimentierten Manganhydroxyd abgesogen, der Niederschlag mit $2 \mathrm{ml}$ reiner konzentrierter Salzsäure und einer Spatelspitze Kaliumiodid p.a. gelöst. Um eine Störung der Analyse durch den Luftsauerstoff auszuschliessen, wurde unter Stickstoffatmosphäre mit n/10-Na-Thiosulfatlösung gegen farblos titriert. Als Indikator wurde 0.5 prozentige Stärkelösung verwendet.

pH-Wert: Der pH-Wert wurde durch elektrometrische Messung bestimmt. (pH-Gerät Typ PHM24 Radiometer mit Glaselektrode.)

Karbonathärte: Nach Vorschrift im Schweizerischen Lebensmittelbuch (4. Auflage), modifiziert nach BERGER (mündliche Mitteilung). Während der Titration wurde Stickstoff durch die Probe geleitet. Dadurch erreicht man eine gute Durchmischung von Wasserprobe und zugegebener Salzsäure, und die entstandene Kohlensäure wird sofort ausgetrieben. Durch diese Versuchsanordnung ist es möglich, den Endpunkt der Titration genauer zu erfassen. Zudem kann das Auskochen der Probe, das sonst zur genauen Ermittlung des Endpunkts nötig ist, umgangen werden.

Gesamtkohlenstoff: Der Gehalt an anorganischem Kohlenstoff (Kohlensäure, Bikarbonat, Karbonat) wurde aus pH-Wert und SBV nach HARveY und RodHE [29] berechnet.

Phosphat: Bestimmung nach Ammoniummolybdat-Zinnchlorid-Methode nach OHLE [30], modifiziert nach AмвüHL und Schmid [31].

Gesamtphosphor: Nach der durch OHLE [30] eingeführten und durch ScHmid und AмBÜHL [32] verfeinerten Methode mit nassem Aufschluss mit Schwefelsäure und Wasserstoffsuperoxyd als Oxydationsmittel, anschliessender Neutralisation mit Ammoniak und Bestimmung nach Phosphatmethode.

Nitrat: Salicylatmethode nach MüLler und WidemanN [33]. 


\subsection{Biologische Untersuchungsmethoden}

Qualitative und quantitative Planktonuntersuchungen:

$50 \mathrm{ml}$ der mit Jod-Jodkali fixierten Wasserproben wurden in mit Deckglas versehene Röhrenverbundkammern gefülllt. Das sedimentierte Plankton wurde nach 48stündiger Absetzzeit im Umkehrmikroskop nach UTERMöHL [27] ausgezählt.

Messung der Primärproduktion:

Nach Steeman-Nielsen [35] wurden etwa $100 \mathrm{~cm}^{3}$ Probenwasser mit etwa $8 \mu \mathrm{C}$ einer sterilen Lösung von $\mathrm{NaHCO}_{3}$ versetzt und während 4 Stunden in horizontaler Lage in situ oder im Rotationslichtthermostaten exponiert. In jedem Messprofil wurde in zwei Tiefenstufen $(1,0 \mathrm{~m}$ und $10,0 \mathrm{~m})$ auch die Dunkelassimilation gemessen. Hierzu wurden die Probeflaschen in Aluminiumfolie verpackt. Dadurch kann eine Erwärmung der Dunkelproben durch Lichtabsorption weitgehend vermieden werden. Nach 4 Stunden wurden $25 \mathrm{ml}$ Probenwasser filtriert (Göttinger Membranfilter MF 30) und die Filter mit «Rubber Cement» in ein Aluminiumschälchen geklebt. Um adsorbiertes, anorganisches $\mathrm{C}^{\mathbf{1 4}}$ auszutreiben, wurden die Zählschälchen während 20 Minuten über rauchender Salzsäure exponiert und nachher bis zur Messung der Aktivität über Blaugel im Exsikkator aufbewahrt. Die Ampullenaktivität wurde mit der Bariumkarbonatmethode bestimmt und die Impulsfrequenz der Schichtdicke $0 \mathrm{mg} / \mathrm{cm}^{2}$ empirisch ermittelt [36]. Die Aktivität der $\mathrm{C}^{14}$-Proben wurde mit dem Methandurchflusszähler FH407 der Firma Frieseke und Höpfner gemessen. Bei der Berechnung der Assimilationsraten wurde ein Korrekturfaktor von 1,06 für den Isotopeffekt berücksichtigt.

\section{Seeuntersuchungen}

\subsection{Lage und Orographie des untersuchten Sees}

Die Horwer Bucht, ein Becken des stark gegliederten Vierwaldstättersees, liegt $5,5 \mathrm{~km}$ südlich Luzern auf $47^{\circ}$ nördlicher Breite und $8^{\circ} 18^{\prime} \mathrm{E}$ von Greenwich. Das annähernd rechteckige Becken von $1 \times 1,5 \mathrm{~km}$ Uferlänge ist maximal $72 \mathrm{~m}$ tief, und sein Volumen beträgt 7,2 $10^{7} \mathrm{~m}^{3}$. Bei einer Seeoberfläche von $1,69 \mathrm{~km}^{2}$ ergibt sich eine mittlere Tiefe $V / 0$ von $42,6 \mathrm{~m}$. Das Volumen der trophogenen Schicht $(0-10 \mathrm{~m})$

Tab. 1.

\begin{tabular}{|c|c|c|c|c|}
\hline \multicolumn{2}{|c|}{ Isobathenflächen $\left[\mathrm{km}^{2}\right]$} & \multicolumn{2}{|c|}{ Volumen $\left.\left[\mathrm{m}^{3} 10^{7}\right]^{\mathbf{1}}\right)$} & \multirow{2}{*}{$\frac{\text { Volumen }[\%]}{22,2}$} \\
\hline $0 \mathrm{~m}$ & 1,69 & $0-10 \mathrm{~m}$ & 1,590 & \\
\hline $14 \mathrm{~m}$ & 1,43 & $10-20 \mathrm{~m}$ & 1,405 & 19,6 \\
\hline $24 \mathrm{~m}$ & 1,25 & $20-30 \mathrm{~m}$ & 1,272 & 17,7 \\
\hline $34 \mathrm{~m}$ & 1,23 & $30-40 \mathrm{~m}$ & 1,140 & 15,9 \\
\hline $44 \mathrm{~m}$ & 0,92 & $40-50 \mathrm{~m}$ & 0,896 & 12,4 \\
\hline $54 \mathrm{~m}$ & 0,68 & $50-60 \mathrm{~m}$ & 0,604 & 8,4 \\
\hline $64 \mathrm{~m}$ & 0,32 & $60-70 \mathrm{~m}$ & $\left.0,278^{2}\right)$ & 3,8 \\
\hline
\end{tabular}

1) Berechnet durch lineare Interpolation [2) Extrapolation] der Isobathenflächen nach der Formel $V=(A+B+A B) H / 3$ wobei $A$ die Oberfläche, $B$ die Unterfläche und $H$ die Höhe der betreffenden Schicht bedeutet. 
verhält sich zu jenem der tropholytischen Schicht wie 1:3,5. Aufgrund dieser Daten müsste es sich nach THIENEMANN [37], abgesehen von den heute allerdings massiven menschlichen Einflüssen, um ein ursprünglich oligotrophes Gewässer handeln.

\subsection{Physikalische Untersuchungen}

\subsubsection{Temperatur}

Die Wassertemperatur beeinflusst das planktische Leben im Verlaufe eines Jahres in vielfacher Weise. Sie bestimmt die Assimilations- und Dissimilationsgeschwindigkeiten (RGT-Regel) und beeinflusst durch die Veränderung der inneren Reibung die Sinkgeschwindigkeit des Planktons. Gegeben durch den Zusammenhang von Temperatur und spezifischem Gewicht bestimmt sie den Grad der Schichtungsstabilität und nimmt damit auf die Nährstoffversorgung der trophogenen Schicht wesentlichen Einfluss.

In Abb. 2 ist der Gang der Temperatur während der Untersuchungsperiode festgehalten. Die höchsten Temperaturen an der Oberfläche wurden im August, die niedrigsten im Februar gemessen. Über dem Grund lagen sie während der ganzen Zeit um rund $4,5^{\circ} \mathrm{C}$. Ausgeprägte inverse Schichtungen konnten nie festgestellt werden.

\subsubsection{Lichtmessungen}

Wenn monochromatisches Licht eine Wasserschicht der Dicke $x$ passiert, verringert sich seine Intensität nach der Gleichung:

$$
I_{x}=I_{0} \cdot e^{-k x},
$$

wobei $k$ vertikaler Extinktionskoeffizient ist. Wird die Lichtintensität im halblogarithmischen Maßstab gegen die Tiefe aufgetragen, so ergibt sich deshalb eine Gerade. Um die optischen Eigenschaften eines homogenen Gewässers zu beschreiben, genügt es daher, neben der Oberflächenintensität $(=100 \%)$ für jeden Spektralbereich noch je einen Punkt zu fixieren. In Tab. 2 sind für die verschiedenen Daten jene Tiefen zusammengestellt, in welchen für die entsprechenden Spektralbereiche $10 \%$ der Oberflächenintensität registriert wurde. Unterhalb dieser $10 \%$-Grenze konnte in einigen Fällen ein von obiger Gleichung abweichendes Verhalten beobachtet werden. Mit diesen «Abnormitäten», die z.T. mit einer Veränderung der Algendichte erklärt werden können, wird sich ZIMmERMANN [66] auseinandersetzen.

Aus Tab. 2 ist ersichtlich, dass das grüne Licht während des ganzen Jahres am tiefsten ins Wasser eindrang.

Tab. 2. Tiefen, in welchen für die verschiedenen Spektralbereiche noch $10 \%$ der Oberflächenintensität $\left(I_{0,05}\right)$ gemessen wurde (Angaben in $\mathrm{m}$ ).

13.6. 15. 7. 14.8. 20.9. 20.10.17.11. 13.12. 11. 1. 17.2. 17.4. 17.5. 14.6. 14. 7. 15.8.

\begin{tabular}{|c|c|c|c|c|c|c|c|c|c|c|c|c|c|c|}
\hline BG12 (blau) & 2,9 & 4,8 & 2,6 & 3,1 & 2,5 & 5,9 & 6,4 & 8,2 & 7,4 & 8,0 & 3,3 & 4,2 & 4,3 & 2,1 \\
\hline VG9 (grün) & 4,9 & 7,2 & 4,3 & 4,9 & 4,0 & 8,3 & 8,8 & 10,6 & 10,2 & 12,3 & 5,7 & 8,8 & - & 6,9 \\
\hline $\mathrm{RG} 2$ (rot) & 2,9 & 3,2 & 2,5 & 3,0 & 2,3 & 3,2 & 3,8 & 4,2 & 4,4 & 4,5 & 3,0 & 4,0 & 3,6 & 3,0 \\
\hline
\end{tabular}




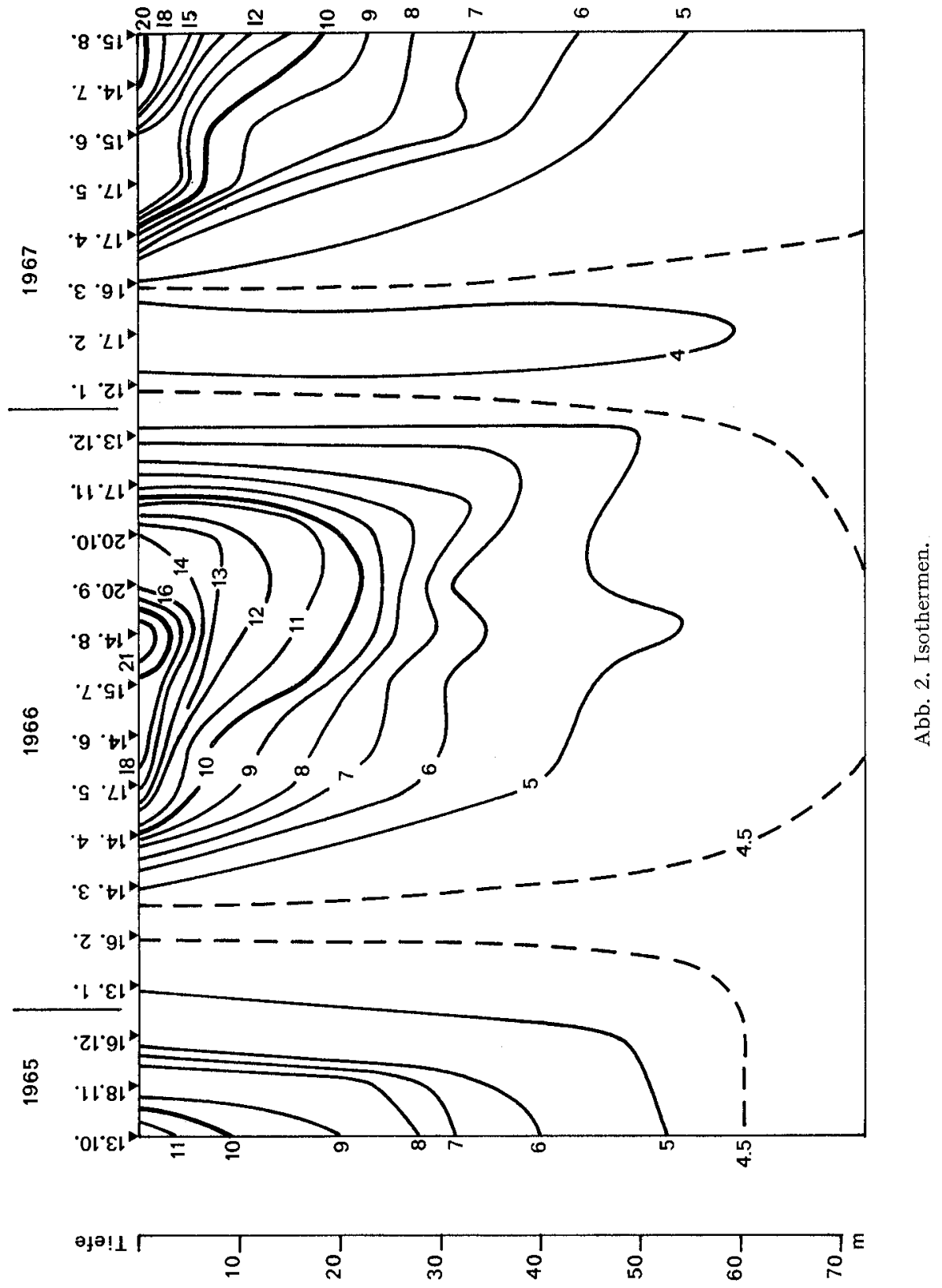




\subsection{Chemische Untersuchungen}

\subsubsection{Sauerstoff}

Die in der trophogenen Schicht produzierte organische Substanz wird während der Sedimentation ganz oder teilweise mineralisiert. Da dieser Prozess Sauerstoff benötigt, während der Stagnation aber keiner durch die Oberfläche ins Hypolimnion eingetragen wird, ist die hypolimnische Sauerstoffzehrung bei Berücksichtigung der Temperatur- und Lichtverhältnisse, der Sinkgeschwindigkeit des Detritus, der Bekkenform und der Hydrographie des Sees sowie des Verhältnisses von autochthoner zu allochthoner organischer Substanz ein relatives Mass für die Primärproduktion und damit indirekt für die Nährstoffversorgung des Epilimnions. Um den Zusammenhang zwischen Primärproduktion und hypolimnischer Sauerstoffzehrung quantitativ studieren zu können, müsste zudem noch jener unbekannte Teil an organischer Substanz erfasst werden, der bereits im Epilimnion dauernd oxydiert und reassimiliert wird, ohne die Sauerstoffzehrung im Hypolimnion zu beeinflussen. Das hypolimnische Sauerstoffdefizit am Ende einer Stagnationsperiode darf daher nicht als absolutes Mass für den Trophiegrad eines Gewässers gewertet werden, ermöglicht aber doch eine erste, rein qualitative Orientierung über seine Belastung mit organischer Substanz. Sowohl im Jahre 1965 als auch 1966 wurden die niedrigsten hypolimnischen Sauerstoffkonzentrationen im Oktober registriert (Abb. 3). Etwa $1 \mathrm{~m}$ über dem Grund

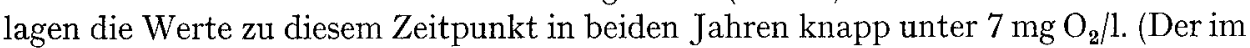
September 1966 gefundene Wert von 5,7 mg $\mathrm{O}_{2} / 1$ kann nur damit erklärt werden, dass die Probe etwas tiefer als normal entnommen wurde.) Aus der Tatsache, dass in März in der gleichen Tiefe in beiden Jahren mehr als $10 \mathrm{mg} \mathrm{O}_{2} / 1$ gemessen wurde, kann geschlossen werden, dass die Zirkulation im Spätherbst und Winter bis zum Grunde vordrang. Die Sauerstoffzehrung über dem Grund betrug im Jahre 1966 rund $4 \mathrm{mg} \mathrm{O}_{2} / 1$ oder $40 \%$ des maximal erreichten Gehaltes. Die höchsten Sauerstoffkonzentrationen über dem Grund wurden in den Monaten Februar bis April gemessen, jedoch wurde die theoretische Sättigungskonzentration, die bei $4,5^{\circ} \mathrm{C}$ und $437 \mathrm{~m}$ Meereshöhe nach Schmassmann [38] 12,4 $\mathrm{mg} / \mathrm{l}$ beträgt, über dem Grund nie erreicht, so dass der See bereits mit einem Sauerstoffdefizit in die Stagnationsperiode eintrat.

In seiner klassischen Arbeit über den Sauerstoffgehalt im eutrophen und oligotrophen See führte THIENEMANN [37] folgende Grössen zur Charakterisierung eines Gewässers ein :

$\mathrm{O}_{2} H / E$ mittlere Sauerstoffkonzentration des Hypolimnions $\times$ Volumen

des Hypolimnions

mittlere Sauerstoffkonzentration des Epilimnions $\times$ Volumen

des Epilimnions

$\Delta H$ (Sättigungskonzentration des Hypolimnions bei der mittleren aktuellen Temperatur) -

(mittlere aktuelle Sauerstoffkonzentration des Hypolymnions)

E Epilimnion = Wasserschicht von 0-10 m Tiefe

$H \quad$ Hypolimnion $=$ Wasserschicht von $10 \mathrm{~m}$ bis zum Grund. 


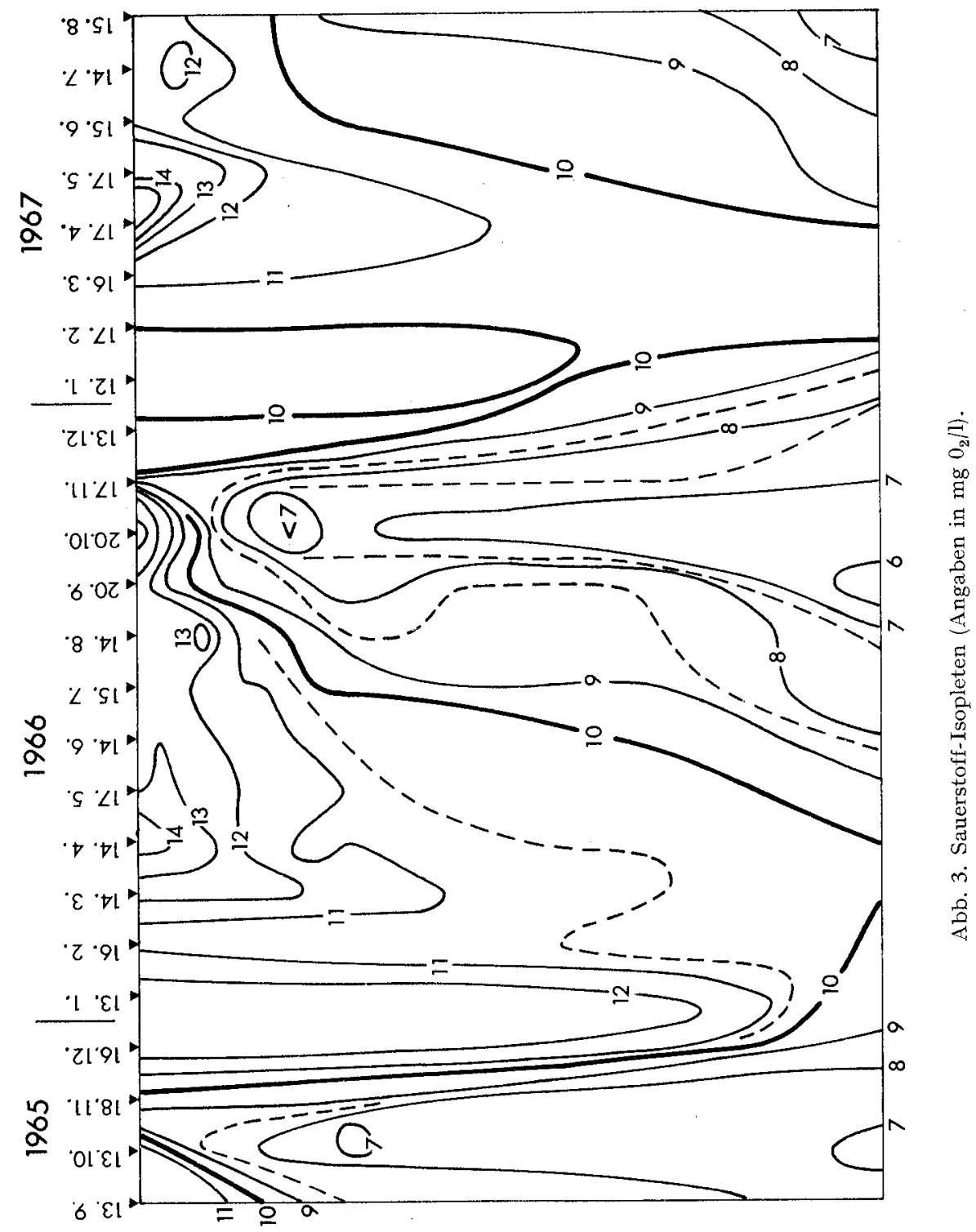

มرa!n 
Als Zeitpunkt zur Beurteilung wird der Hochsommer (maximal ausgeprägte Sommerstagnation) gewählt.

Bei einem echt oligotrophen See ist der Ausdruck $\mathrm{O}_{2} \mathrm{H} / \mathrm{E}$ im Hochsommer grösser als 1 , und $\Delta H$ ist klein. Beim echt eutrophen See dagegen liegen die Werte für $\mathrm{O}_{2} H / E$ unter 1 , und $\Delta H$ ist gross.

In den Jahren 1965-1967 wurden für die Horwer Bucht die folgenden Werte ermittelt:

Tab. 3.

\begin{tabular}{|c|c|c|c|}
\hline Datum & $\Delta H$ & $\mathrm{O}_{2} H / E$ & $\begin{array}{l}\mathrm{O}_{2} \text {-Gehalt der } \\
\text { grössten Tiefe }\end{array}$ \\
\hline 13. 9. 1965 & $3,1 \mathrm{mg} \mathrm{O} / 1$ & 2,7 & $7,3 \mathrm{mg} \mathrm{O} / 1$ \\
\hline 14. 8. 1966 & $2,9 \mathrm{mg} \mathrm{O}_{2} / 1$ & 2,1 & $7,6 \mathrm{mg} \mathrm{O} / 1$ \\
\hline 14. 8.1967 & $3,4 \mathrm{mg} \mathrm{O}_{2} / 1$ & 2,6 & $6,4 \mathrm{mg} \mathrm{O} / 1$ \\
\hline
\end{tabular}

Nach Å BerG und Rodhe [39] ist der Verlauf der Sauerstoffkurven als klinograd bis leicht minus-heterograd zu bezeichnen. Das lässt in unsern Breiten von einigen Spezialfällen (humose und meromiktische Seen, Seen mit kleiner mittlerer Tiefe) auf einen See mit eutrophem Charakter schliessen.

$\mathrm{Zu}$ Vergleichszwecken seien hier noch einige Werte der von ThIEnEmann [37] untersuchten norddeutschen Seen zitiert:

Tab. 4.

\begin{tabular}{|c|c|c|c|}
\hline & $\Delta H$ & $\mathrm{O}_{2} H / E$ & $\begin{array}{l}\mathrm{O}_{2} \text {-Gehalt der } \\
\text { grössten Tiefe }\end{array}$ \\
\hline Oligotrophe Seen & $2,18-3,84 \mathrm{mg} \mathrm{O}_{2} / 1$ & $1,95-1,54$ & $7,6-8,6 \mathrm{mg} \mathrm{O}_{2} / 1$ \\
\hline Übergangsseen . & $3,32-7,30 \mathrm{mg} \mathrm{O} / 1$ & $1,22-0,34$ & $4,0-8,6 \mathrm{mg} \mathrm{O} \mathrm{O}_{2} / 1$ \\
\hline eutrophe Seen & $5,48-8,82 \mathrm{mg} \mathrm{O}_{2} / \mathrm{l}$ & $0,45-0,08$ & $1,4-5,7 \mathrm{mg} \mathrm{O} / 1$ \\
\hline
\end{tabular}

Obwohl die Werte für die $\mathrm{O}_{2}-H / E$-Quotienten dank den günstigen morphometrischen Verhältnissen noch in den oligotrophen Bereich fallen, deuten die Sauerstoffkonzentrationen über dem Grund, die $\Delta H$-Werte und namentlich der generelle Verlauf der Sauerstoffkurven während der Stagnationsperiode darauf hin, dass dieser Seeteil als «Übergangssee» in der Skala oligotroph-eutroph irgendwo zwischen diesen beiden Polen einzuordnen ist.

\subsubsection{Stickstoff}

Unter dem Nährelementen kommt dem Stickstoff als Baustein für sämtliche Eiweisse eine überragende Rolle zu. Im Seewasser kann er elementar, als Nitrat, Nitrit oder Ammonium vorliegen. Elementarer Stickstoff kann durch gewisse Blaualgen und Bakterien assimiliert werden (s. S. 3). Die Annahme, dass in Seen ohne starken Sauerstoffschwund das Nitrat neben gelegentlichen Spuren von Ammonium und Nitrit von der Oberfläche bis zum Grund die hauptsächlichste anorganische Stickstoffverbindung ist, wurde durch langjährige periodische Seewasserkontrollen der EAWAG an andern Stellen im Vierwaldstättersee eindeutig bestätigt. In der vorliegenden Arbeit wurde daher nur diese Komponente berücksichtigt. Die erhaltenen 
Analysenresultate sind in Tab. 5 zusammengestellt. Es geht daraus hervor, dass die mittlere Konzentration während der Vollzirkulation rund $0,4 \mathrm{mg} \mathrm{NO}_{3}-\mathrm{N} / \mathrm{l}$ beträgt. $\mathrm{Ab}$ April setzte im Epilimnion eine deutliche Zehrung ein, die die Konzentration an Nitratstickstoff bis zum Ende der Sommerstagnation in den obersten Wasserschichten bis auf $0,05 \mathrm{mg} / 1$ absinken liess. VOLLENWEIdER [40] untersuchte den Zusammenhang zwischen dem Trophiegrad und der Konzentration an mineralischem Stickstoff zur Zeit der Zirkulation. In seiner Skala wäre der untersuchte Seeteil aufgrund seines Nitratgehalts als oligomesotrophes bis mesoeutrophes Gewässer zu bezeichnen.

Tab. 5. Nitratkonzentrationen (Angaben in $\mathrm{mg} \mathrm{NO}_{3}-\mathrm{N} / \mathrm{l}$ ).

\begin{tabular}{|c|c|c|c|c|c|c|c|c|c|c|c|}
\hline $\begin{array}{l}\text { Tiefe } \\
{[\mathrm{m}]}\end{array}$ & $\begin{array}{l}1965 \\
13.9 .\end{array}$ & 13.10 & 17.11 & $\begin{array}{l}1966 \\
13.1 .\end{array}$ & 16. 2. & 14.3. & 14.4 & 17.5 & 13.6. & 14.8 & 20.9 \\
\hline 0,0 & 0,20 & 0,15 & 0,23 & 0,37 & 0,32 & 0,33 & 0,21 & 0,15 & 0,15 & 0,10 & 0,05 \\
\hline 2,5 & 0,20 & 0,17 & 0,23 & 0,36 & 0,32 & 0,32 & 0,20 & 0,22 & 0,20 & 0,06 & 0,05 \\
\hline 5,0 & 0,21 & 0,26 & 0,24 & 0,26 & 0,37 & 0,32 & 0,23 & 0,26 & 0,23 & 0,12 & $0,1.1$ \\
\hline 7,5 & 0,20 & 0,27 & 0,24 & 0,36 & 0,32 & 0,34 & 0,31 & 0,27 & 0,25 & 0,10 & 0,15 \\
\hline 10,0 & 0,24 & 0,27 & 0,24 & 0,37 & 0,32 & 0,32 & 0,34 & 0,28 & 0,26 & 0,16 & 0,19 \\
\hline 12,5 & 0,25 & 0,29 & 0,24 & 0,36 & 0,35 & 0,33 & 0,36 & 0,32 & 0,26 & 0,18 & 0,20 \\
\hline 15,0 & 0,27 & 0,32 & 0,25 & 0,37 & 0,35 & 0,34 & 0,36 & 0,32 & 0,27 & 0,20 & 0,25 \\
\hline 20,0 & 0,35 & 0,33 & 0,24 & 0,37 & 0,35 & 0,36 & 0,40 & 0,34 & 0,33 & 0,29 & 0,29 \\
\hline 30,0 & 0,40 & 0,37 & 0,35 & 0,37 & 0,35 & 0,38 & 0,39 & 0,41 & 0,38 & 0,37 & 0,42 \\
\hline 50,0 & 0,40 & 0,38 & 0,38 & 0,37 & 0,35 & 0,40 & 0,39 & 0,45 & 0,41 & 0,40 & 0,44 \\
\hline 71,0 & 0,40 & 0,40 & 0,39 & 0,40 & 0,37 & 0,42 & 0,42 & 0,45 & 0,41 & 0,45 & 0,44 \\
\hline $\begin{array}{l}\text { Tiefe } \\
{[\mathrm{m}]}\end{array}$ & $\begin{array}{l}1966 \\
20.10\end{array}$ & 17.11 & 13. 12 & $\begin{array}{l}1967 \\
11.1 .\end{array}$ & 17.2. & 16. 3. & 17.4 & 17. 5 . & 14.6. & 14.7. & 15.8. \\
\hline 0,0 & 0,05 & 0,19 & 0,33 & 0,33 & 0,33 & 0,36 & 0,18 & 0,17 & 0,24 & 0,18 & 0,20 \\
\hline 2,5 & 0,05 & 0,19 & 0,32 & 0,34 & 0,33 & 0,36 & 0,17 & 0,17 & 0,24 & 0,26 & 0,10 \\
\hline 5,0 & 0,05 & 0,19 & 0,32 & 0,34 & 0,36 & 0,35 & 0,33 & 0,17 & 0,27 & 0,24 & 0,19 \\
\hline 7,5 & 0,21 & 0,20 & 0,32 & 0,34 & 0,34 & 0,39 & 0,31 & 0,23 & 0,27 & 0,30 & 0,21 \\
\hline 10,0 & 0,28 & 0,20 & 0,32 & 0,33 & 0,35 & 0,38 & 0,33 & 0,26 & 0,27 & 0,31 & 0,13 \\
\hline 12,5 & 0,33 & 0,24 & 0,34 & 0,33 & 0,35 & 0,38 & 0,33 & 0,26 & 0,30 & 0,35 & 0,21 \\
\hline 15,0 & 0,37 & 0,32 & 0,33 & 0,34 & 0,36 & 0,38 & 0,33 & 0,29 & 0,30 & 0,36 & 0,27 \\
\hline 20,0 & 0,43 & 0,33 & 0,35 & 0,33 & 0,35 & 0,38 & 0,33 & 0,35 & 0,36 & 0,39 & 0,36 \\
\hline 30,0 & 0,46 & 0,36 & 0,32 & 0,35 & 0,36 & 0,39 & 0,33 & 0,38 & 0,37 & 0,39 & 0,36 \\
\hline 50,0 & 0,47 & 0,36 & 0,41 & 0,35 & 0,35 & 0,39 & 0,38 & 0,43 & 0,40 & 0,42 & 0,36 \\
\hline 71,0 & 0,45 & 0,36 & 0,43 & 0,36 & 0,38 & 0,40 & 0,38 & 0,40 & 0,40 & 0,43 & 0,35 \\
\hline
\end{tabular}

\subsubsection{Phosphat}

Neueste Untersuchungen von RIGLER [41] lassen es als nicht ausgeschlossen erscheinen, dass die nach der Methode von AmвüHL und Schmid [31] bestimmten aktuellen Orthophosphatkonzentrationen trotz ihrer hohen analytischen Präzision zu hoch ausgefallen sind, weil leicht hydrolysierbare Phosphorverbindungen in schwefelsaurer Lösung möglicherweise schon bei Zimmertemperatur hydrolysiert werden. Es ist ferner denkbar, dass durch das Platzen von Organismen während der Filtration Phosphorverbindungen ins Filtrat gelangen und durch Phosphatasen abgebaut werden (s. S. 17). Leider wurden diese vermuteten Störeinflüsse bis heute 
noch nicht systematisch untersucht. Da im Sommer aber bei relativ hohen Konzentrationen an partikulärem und gelöstem Phosphor oft $0,0 \mu \mathrm{g} \mathrm{PO}_{4}-\mathrm{P} / 1$ gemessen wird (bei einer Genauigkeit von $\pm 0,2 \mu \mathrm{g} \mathrm{PO}_{4}-\mathrm{P} / 1$ ), darf angenommen werden, dass solche Artefakte zumindest nur eine untergeordnete Rolle spielen.

STRICKLAND und Parsons [42] schlagen vor, die oben als $\mathrm{PO}_{4}-\mathrm{P} / 1$ bezeichnete P-Fraktion mit «soluble reactive phosphorus» $z u$ bezeichnen. In dieser Arbeit wird sie jedoch, wie es im deutschen Sprachgebiet noch allgemein üblich ist, trotz den erwähnten Einwänden als Phosphat $\left(\mathrm{PO}_{4}-\mathrm{P}\right)$ bezeichnet. Der generelle Verlauf der Konzentrationsänderungen dieser Phosphorkomponente im Verlauf des Jahres wurde in Abb. 4 dargestellt. (Die exakten Einzelwerte müssen der Tab. 6 entnommen werden.) Vollständig homogene Verteilung konnte niemals, auch nicht während der Zirkulationsperioden, beobachtet werden. In den Monaten März/April setzte in guter Übereinstimmung mit der Nitratzehrung eine gleichmässige, massive Phosphatzehrung ein, die von der Oberfläche bis in grosse Tiefen reichte. Ein Vergleich mit Abb. 2 zeigt, dass diese scheinbar von der Tiefe und damit von der autotrophen Lebensweise der planktischen Algen unabhängige Zehrung durch Zirkulationsströmungen ermöglicht wurde. Nach dem Unterschreiten der 2,5- $\mu$ g- $\mathrm{PO}_{4}-\mathrm{P} / 1-\mathrm{Grenze}$ im April war die weitere Abnahme der Phosphatkonzentration deutlich verlangsamt.

Tab. 6. Phosphatkonzentrationen (Angaben in $\mu \mathrm{g} \mathrm{PO}_{4}-\mathrm{P} / \mathrm{l}$ ).

\begin{tabular}{|c|c|c|c|c|c|c|c|c|c|c|c|c|}
\hline $\begin{array}{l}\text { Tiefe } \\
{[\mathrm{m}]}\end{array}$ & $\begin{array}{l}1965 \\
13.9 .\end{array}$ & 13.10 & 17.11 & 16.12. & $\begin{array}{l}1966 \\
13.1\end{array}$ & 16. 2. & 14. 3. & 14.4 & 17. 5 . & 13.6. & 15.7. & 14. 8. \\
\hline 0,0 & 1,0 & 0,5 & 1,8 & 2,4 & 5,2 & 5,0 & 5,8 & 1,4 & 2,2 & 2,4 & 0,6 & 0,0 \\
\hline 2,5 & 0,8 & 0,7 & 1,7 & 1,0 & 5,2 & 5,0 & 6,0 & 1,4 & 2,0 & 1,2 & 0,6 & 0,2 \\
\hline 5,0 & 0,8 & 0,2 & 1,4 & 2,6 & 5,2 & 5,0 & 7,2 & 1,3 & 1,9 & 1,2 & 0,6 & 0,8 \\
\hline 7,5 & 0,9 & 0,5 & 1,5 & 1,8 & 5,2 & 5,8 & 6,0 & 1,0 & 1,2 & 1,9 & 0,6 & 0,8 \\
\hline 10,0 & 0,9 & 0,4 & 1,1 & 1,7 & 5,8 & 5,8 & 6,4 & 1,2 & 1,5 & 1,1 & 0,6 & 0,8 \\
\hline 12,5 & 1,0 & 0,9 & 2,0 & 2,4 & - & - & 5,8 & 1,1 & 1,3 & 1,0 & 0,6 & 0,2 \\
\hline 15,0 & 1,0 & 1,5 & 1,9 & 4,1 & - & 4,6 & 6,0 & 0,6 & 1,3 & 0,7 & 0,3 & 0,2 \\
\hline 20,0 & 2,2 & 0,7 & 1,9 & 2,6 & 6,0 & 5,2 & 6,6 & 2,0 & 1,3 & 1,3 & 0,9 & 0,4 \\
\hline 30,0 & - & 0,9 & 3,9 & 3,4 & 6,0 & 5,5 & 7,8 & 3,6 & 3,2 & 3,2 & 1,6 & 0,4 \\
\hline 50,0 & 10,0 & 2,6 & 6,9 & 6,8 & 7,5 & 7,0 & 11,0 & 5,1 & 7,0 & 6,8 & 11,3 & 9,3 \\
\hline 71,0 & 21,6 & 18,6 & 31,1 & 18,2 & 15,0 & 9,3 & 11,8 & 12,6 & 11,5 & 16,0 & 21,6 & 20,0 \\
\hline $\begin{array}{l}\text { Tiefe } \\
{[\mathrm{m}]}\end{array}$ & $\begin{array}{l}1966 \\
20.9\end{array}$ & 20.10 & 17.11 & 13. 12. & $\begin{array}{l}1967 \\
11.1 .\end{array}$ & 17.2 & 16. 3. & 17.4. & 17.5. & 14.6 . & 14.7. & 15. 8. \\
\hline 0,0 & 1,9 & 0,0 & 2,5 & 3,9 & 6,1 & 4,0 & 4,3 & 2,0 & 1,9 & 2,1 & 1,3 & 1,9 \\
\hline 2,5 & 1,9 & 0,0 & 2,5 & 3,5 & 6,0 & 4,0 & 4,6 & 1,5 & 2,6 & 1,3 & 0,4 & 1,9 \\
\hline 5,0 & 1,8 & 0,4 & 2,5 & 3,5 & 6,0 & 4,1 & 4,6 & 2,0 & 1,8 & 2,1 & 0,2 & 0,9 \\
\hline 7,5 & 0,9 & 0,6 & 3,0 & 3,5 & 6,0 & 4,1 & 5,7 & 3,0 & 2,3 & 4,4 & 0,1 & 1,2 \\
\hline 10,0 & 0,9 & 1,5 & 3,0 & 3,5 & 6,0 & 4,0 & 5,4 & 3,0 & 2,6 & 4,4 & 0,3 & 1,1 \\
\hline 12,5 & 0,4 & 2,6 & 4,0 & 3,5 & 6,0 & 4,0 & 6,5 & 3,0 & 1,5 & 4,4 & 0,0 & 1,2 \\
\hline 15,0 & 0,6 & 3,0 & 6,0 & 3,5 & 6,0 & 4,0 & 6,1 & 3,0 & 1,5 & 5,1 & 0,2 & 0,3 \\
\hline 20,0 & 0,6 & 3,9 & 6,5 & 3,5 & 6,0 & 3,8 & 9,8 & 3,0 & 4,9 & 5,0 & 0,7 & 1,4 \\
\hline 30,0 & 1,4 & 5,4 & 8,3 & 3,6 & 6,2 & 4,0 & 9,5 & 4,0 & 5,8 & 6,5 & 6,2 & 2,6 \\
\hline 50,0 & 7,0 & 7,6 & 12,3 & 11,5 & 6,1 & 4,1 & 8,9 & 9,0 & 13,3 & 9,6 & 10,8 & 13,2 \\
\hline 71,0 & 46,0 & 25,4 & 19,5 & 20,0 & 6,9 & 7,6 & 8,9 & 11,0 & 18,0 & 16,9 & 21,6 & 20,8 \\
\hline
\end{tabular}




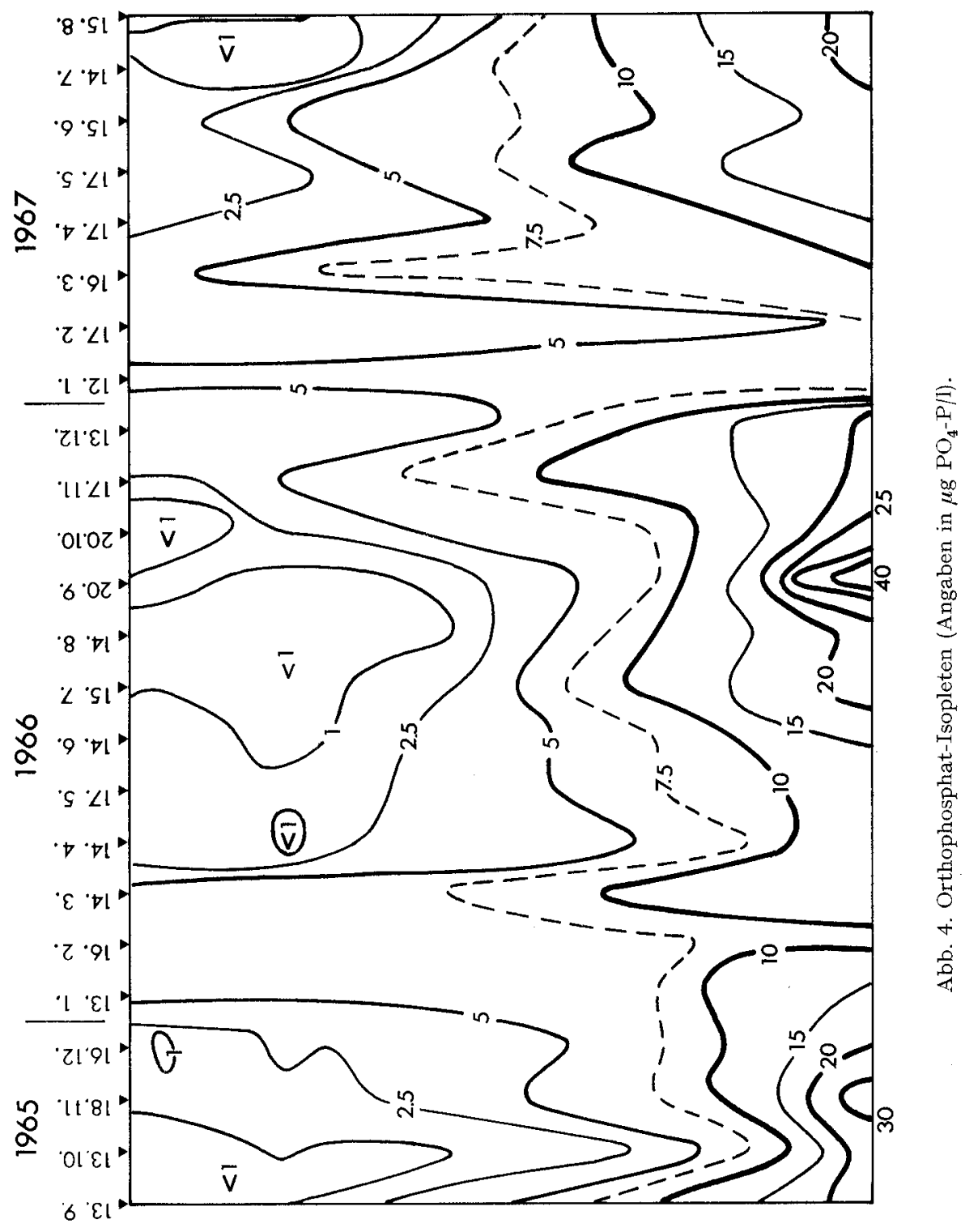

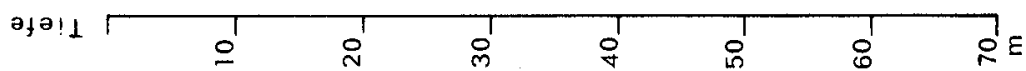


Minimale Konzentrationen von weniger als $1 \mu \mathrm{g} \mathrm{PO}_{4}^{-\mathrm{P}} / 1$ wurden erst im Juli/August erreicht. Dass die Phosphatzehrung auch jetzt noch tiefer als die trophogene Schicht reichte, kann z.T. damit erklärt werden, dass der Vierwaldstättersee auch im Hochsommer keine sehr ausgeprägte thermische Schichtung zeigt und damit eine schwache Teilzirkulation bis in rund $30 \mathrm{~m}$ Tiefe ermöglicht. Anderseits ist damit zu rechnen, dass absinkendes Plankton auch unterhalb des Kompensationspunkts prämortal noch Phosphat zu binden vermag. Der kleine Konzentrationsgradient in der Wasserschicht 0-20 m berechtigt aber zur Annahme, dass die Phosphatzufuhr zur trophogenen Schicht durch turbulente Strömungen und Diffusion, verglichen mit der Belastung durch Abwasser (vgl. S. 24), während des ganzen Sommers vernachlässigt werden kann.

Unmittelbar nach der von der Oberfläche her einsetzenden Phosphatzehrung setzte vom Seegrund her eine deutliche Zunahme der Phosphatkonzentration ein, die im tiefen Hypolimnion im September ihr Maximum erreichte. Erste Messungen von BLösch (unveröffentlicht) (vgl. S. 23) lassen vermuten, dass ein grosser Teil des sedimentierten PP den Seegrund unverändert erreicht und daher während des Absinkens wenig zum Konzentrationsanstieg im Hypolimnion beiträgt. Die fortschreitende Mineralisation am Seegrund entzog sich der Analyse, da keine extrem bodennahen Proben entnommen wurden. Sie erklärt aber den plötzlichen Anstieg der $\mathrm{PO}_{4}$-Konzentration in der gesamten Wassermasse während der Frühjahrszirkulation (März 1966/67). Wie gross die im Sediment jährlich zurückgehaltenen Phosphormengen sind, kann aufgrund der wenigen Sedimentationsmessungen noch nicht beurteilt werden. Angaben von ZüLLIG [2] lassen jedoch vermuten, dass es sich um beträchtliche Mengen handeln kann. Er ermittelte 1954 für die obersten Sedimentschichten die folgenden spezifischen Jahresbelastungen (Rückhalt in $\mathrm{g} / \mathrm{m}^{2}$ pro Jahr):

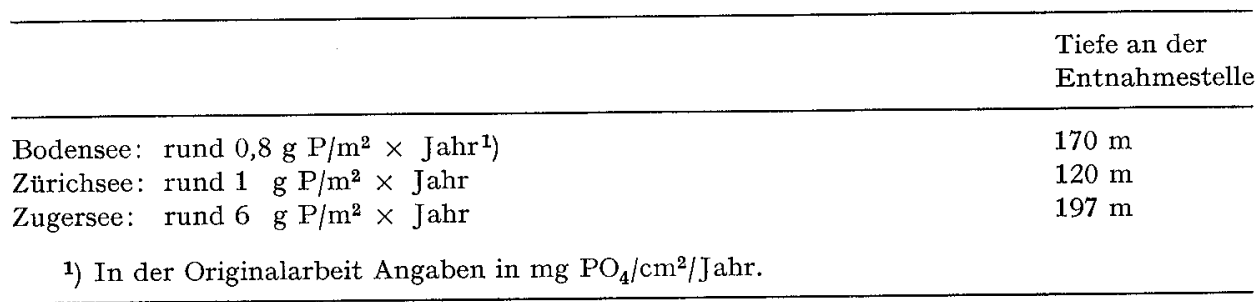

\subsubsection{Gelöste organische Phosphorverbindungen (GOP)}

Neben dem Phosphat werden im Seewasser stets noch andere gelöste Phosphorverbindungen gefunden. Sie werden als Differenz von Gesamtphosphorkonzentration im Filtrat und Phosphatkonzentration berechnet. Viele Autoren nennen diese Komponente "gelöster organischer Phosphor». (engl. DOP), andere "kolloidaler Phosphor» [8]. Da aber allfällige im Wasser gelöste anorganische Polyphosphate mit der Phosphatbestimmungsmethode nicht quantitativ erfasst werden, ist es fraglich, wieweit die Bezeichnung "gelöste organische Phosphorverbindungen" zutrifft. In der von STRICKLAND und PARSONS [42] vorgeschlagenen Bezeichnung «soluble unreactive phosphorus» kommt zum Ausdruck, wie wenig im einzelnen über diese Komponente bekannt ist. 
Die gemessenen Konzentrationen sind in Tab. 7 zusammengefasst. Während der Sommerstagnation fielen sie in der trophogenen Schicht oft etwas höher aus als im Hypolimnion. Wieweit diese Beobachtung reel ist und nicht dadurch zustande kommt, dass feine Planktonorganismen beim Filtrieren platzen und dadurch Phosphorverbindungen als Artefakt ins Filtrat gelangen, muss durch ergänzende methodische Untersuchungen noch abgeklärt werden. RIGLER [43] hat gezeigt, dass die gefundene GOP-Konzentration stark von der Methode abhängt, nach der das Seston vom Wasser getrennt wurde.

Tab. 7. GOP-Konzentrationen (Angaben in $\mu \mathrm{g} \mathrm{P} / \mathrm{l}$ ).

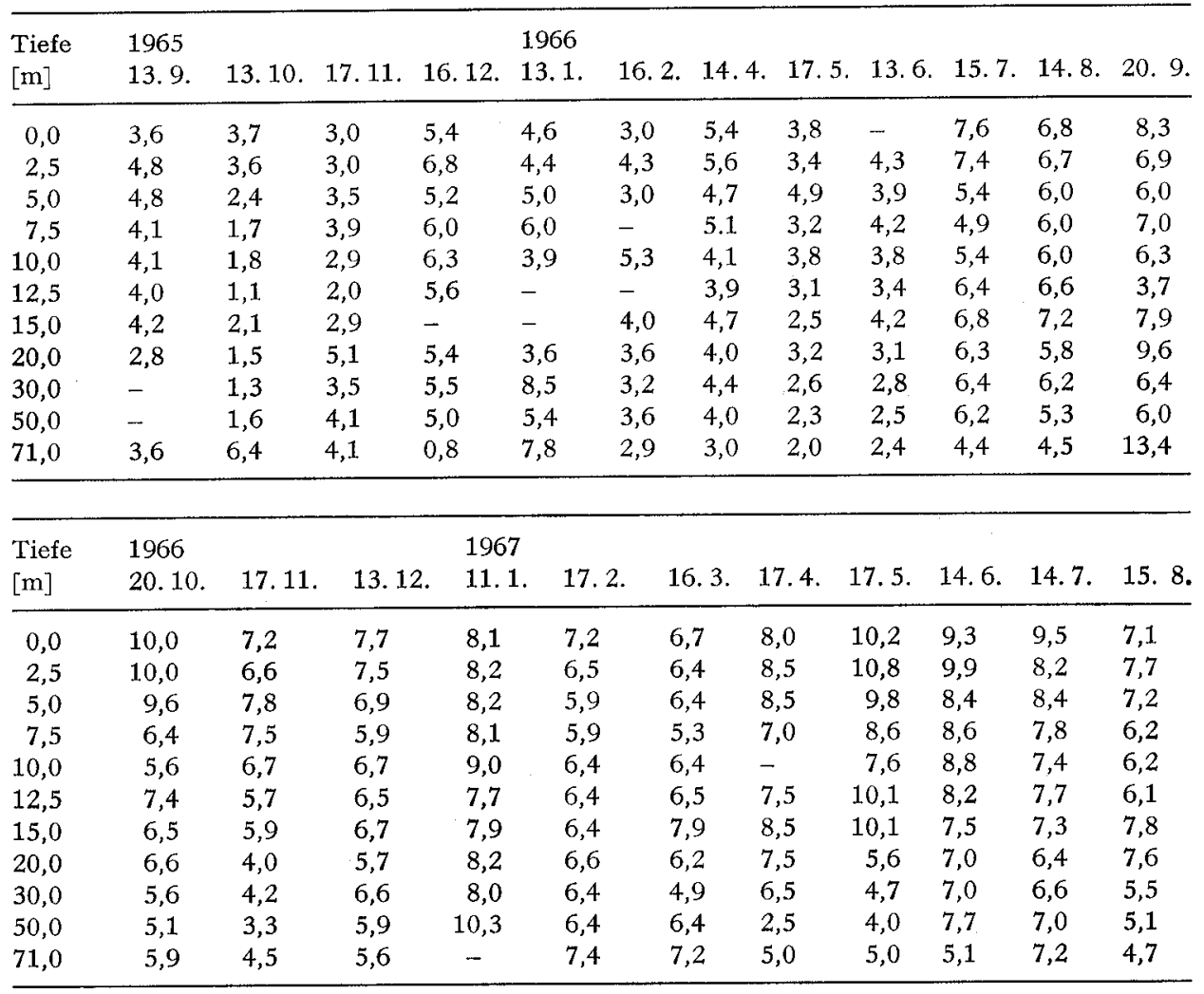

Chu [44], Rodhe [45] und Provasoli [46] haben nachgewiesen, dass verschiedene Algen ihren Phosphorbedarf aus organischen Phosphorverbindungen wie Phytin, Monoäthylphosphat und den Nukleotiden Adenyl-, Guanyl- und Cytidylsäure zu decken vermögen. Galloway und KRAuss [47] verglichen die Wachstumsrate von Chlorella pyrenoidosa in Nährlösungen mit Polyphosphaten verschiedener Kettenlänge als Phosphorquelle $(n=1-55)$. Sie konnten nachweisen, dass $C$. pyrenoidosa Polyphosphate enzymatisch abbaut und sich gleich gut entwickelt, wie wenn Ortophosphat als P-Quelle geboten wird. Obwohl für Scenedesmus quadricauda verschiedene Phosphatasen nachgewiesen wurden, kann diese Alge in bakterienfreier 
Reinkultur gebundene Phosphate nicht als Phosphorquelle ausnützen, weil das Plasmolema für solche Verbindungen offenbar impermeabel ist [48]. OvERBECK zeigte aber, dass Escherichia coli, Bacillus subtilis und ein natürliches Gemisch von Wasserbakterien in der Lage sind, Natriumpyrophosphat und Natriumglycerophosphat zu spalten und so andern Organismen Phosphat zur Verfügung zu stellen.

Da die handelsüblichen Haushaltwaschmittel neben Detergentien auch Polyphosphate enthalten, ist damit im Falle der Einleitung häuslicher Abwässer in den See die direkte düngende Wirkung gegeben, nachdem ForsberG [49] im Experiment die wachstumsfördernde Wirkung verschiedener Waschmittel auf Algen (Microcystis aeruginosa, Anabaena cylindrica, Scenedesmus quadricauda, Selenastrum capricornutum und Ankistrodesmus falcatus) nachgewiesen hat.

Diese wenigen erwähnten Arbeiten deuten darauf hin, dass auch die GOP-Komponente als Phosphorquelle für das Phytoplankton in Betracht zu ziehen ist, zumal OvERBEcK [50] tägliche Konzentrationsschwankungen dieser Komponente in einem kleinen Teich festgestellt hat und WATT und Hayes [51] mit der Tracermethode die Umwandlung von GOP in $\mathrm{PO}_{4}$ und partikuläre Phosphorverbindungen (PP) im Wasser von Peggy's Cove nachweisen konnten. Aus eigenen Versuchen (s. S. 46) muss ebenfalls geschlossen werden, dass GOP in PP übergeführt wurde.

Trotzdem kann bei der unbekannten qualitativen Zusammensetzung der GOPVerbindungen quantitativ wenig darüber gesagt werden, wieweit sie als P-Quelle vom Phytoplankton direkt ausgenützt werden können. Da zugesetztes Orthophosphat vom Phytoplankton während des Sommers aber sehr rasch adsorbiert wird, deuten die gleichzeitig festgestellten hohen GOP-Konzentrationen jedoch darauf hin, dass diese Phosphorfraktion auch bei Berïcksichtigung des bakteriellen Abbaus gesamthaft gesehen wesentlich schlechter ausgenützt wird als das Orthophosphat. Das will aber wiederum nicht heissen, dass nicht einzelne Komponenten des GOP sehr aktiv am limnischen Phosphorkreislauf beteiligt sein können.

\subsubsection{Geformter oder partikulärer Phosphor (PP)}

Die Konzentration dieser Phosphorkomponente ergibt sich als Differenz der Gesamtphosphorkonzentration des Rohwassers und derjenigen des Filtrats. Sie umfasst alle jene Phosphorverbindungen, die mit Partikeln von mehr als $0,5 \mu \mathrm{m}$ Durchmesser assoziiert sind und daher bei der Filtration auf dem Membranfilter MF 50 zurückbleiben.

Die zeitlichen und räumlichen Veränderungen dieser Komponente im Verlauf der Untersuchungsperiode wurden in Abb. 5 dargestellt. Die einzelnen Analysenwerte sind in Tab. 8 zusammengestellt. Punkte, die während der Untersuchungen Konzentrationen von mehr als $10 \mu \mathrm{g} \mathrm{PP} / 1$ aufwiesen, liegen in Abb. 5 in der schraffierten Fläche. Daraus geht deutlich hervor, dass während der Frühjahrszirkulation auch diese Komponente mehr oder weniger homogen über die ganze Tiefe verteilt wurde. Im Juni 1966 und im Mai 1967 stieg die PP-Konzentration auch in grösseren Tiefen plötzlich wieder an. Die anschliessende Verarmung der trophogenen Schicht an PP deutet darauf hin, dass dieser Konzentrationsanstieg wohl auf verstärkte Sedimentation zurückzuführen ist. Zwischen dem Durchlaufen dieses Minimums und der einsetzenden Herbstzirkulation, welche die PP-Schichtung mehr und mehr verwischte, konnte in der trophogenen Schicht ein zweites PP-Maximum beobachtet werden. 
Tab. 8. PP-Konzentrationen (Angaben in $\mu \mathrm{g} \mathrm{P} / 1$ ).

\begin{tabular}{|c|c|c|c|c|c|c|c|c|c|c|c|c|}
\hline $\begin{array}{l}\text { Tiefe } \\
{[\mathrm{m}]}\end{array}$ & $\begin{array}{l}1965 \\
13.9 .\end{array}$ & 13.10 & 17.11 & 16.12. & $\begin{array}{l}1966 \\
13.1 .\end{array}$ & 16.2 & 14.4 & 17.5 . & 13.6. & 15.7 & 14.8 & 20. 9. \\
\hline 0,0 & 13,6 & 14,2 & - & 6,6 & 4,6 & 6,2 & 10,8 & 20,9 & 13,5 & 14,8 & 20,6 & 23,0 \\
\hline 2,5 & 14,6 & 11,1 & 13,5 & 5,6 & 4,9 & 4,3 & 17,6 & 14,2 & 17,5 & 15,8 & 17,0 & 23,7 \\
\hline 5,0 & 15,0 & 6,2 & 11,5 & 6,6 & 8,2 & 8,0 & 15,2 & 14,6 & 23,1 & 12,2 & 17,0 & 22,7 \\
\hline 7,5 & 13,5 & 4,2 & 12,7 & 6,6 & 2,8 & - & 14,9 & 18,6 & 13,7 & 8,7 & 30,8 & 16,3 \\
\hline 10,0 & 12,5 & 5,0 & 15,6 & - & 4,8 & 4,9 & 14,5 & 15,3 & 14,6 & 7,4 & 15,1 & 14,4 \\
\hline 12,5 & 6,4 & 7,2 & 14,0 & 7,0 & - & - & 13,2 & 13,2 & 14,8 & 11,0 & 17,0 & 17,7 \\
\hline 15,0 & 6,2 & 5,2 & 12,0 & 7,0 & $\ldots$ & 5,4 & 11,7 & 13,6 & 15,1 & 8,9 & 7,2 & 10,3 \\
\hline 20,0 & 3,0 & 3,6 & 11,6 & 6,8 & 6,6 & 3,2 & 8,8 & 10,1 & 11,0 & 6,8 & 9,2 & 6,6 \\
\hline 30,0 & 6,3 & 3,4 & 5,9 & 6,8 & 6,8 & 5,3 & 7,2 & 7,0 & 10,9 & 4,0 & 2,5 & 5,0 \\
\hline 50,0 & 5,0 & 2,2 & 2,3 & 4,6 & 5,2 & 3,5 & 5,5 & 5,2 & 7,9 & 1,5 & 3,6 & 5,6 \\
\hline 71,0 & $3 ; 6$ & 4,4 & 4,2 & 7,8 & 3,4 & 5,0 & 4,0 & 4,6 & 9,6 & 10,5 & 7,7 & 18,0 \\
\hline $\begin{array}{l}\text { Tiefe } \\
{[\mathrm{m}]}\end{array}$ & $\begin{array}{l}1966 \\
20.10\end{array}$ & 17.11 & 13.12. & $\begin{array}{l}1967 \\
11.1 .\end{array}$ & 17.2. & 16.3 & 17.4 & 17.5 & 14.6. & 14.7 & 14.8 & \\
\hline 0,0 & 18,4 & 14,1 & 11,0 & 5,8 & 9,8 & 11,7 & 20,0 & 14,6 & 11,6 & 11,8 & 14,3 & \\
\hline 2,5 & 19,0 & $15, \mathbf{1}$ & 10,4 & 7,8 & 8,2 & 18,8 & 33,0 & 27,3 & 14,8 & 6,6 & 15,2 & \\
\hline 5,0 & 21,7 & 14,5 & 10,6 & 8,8 & 8,3 & 17,5 & 19,5 & 18,9 & 14,0 & 6,5 & 13,3 & \\
\hline 7,5 & 10,5 & 13,7 & 11,6 & 8,1 & 9,5 & 17,5 & 16,5 & 12,1 & 8,6 & 5,0 & 11,6 & \\
\hline 10,0 & 9,0 & 14,3 & 11,2 & 10,4 & 10,5 & 15,2 & 12,5 & 15,0 & 8,4 & 5,3 & 12,7 & \\
\hline 12,5 & 4,1 & 10,3 & 11,4 & 6,8 & 9,2 & 14,0 & 9,5 & 12,8 & 9,0 & 5,7 & 11,1 & \\
\hline 15,0 & 6,5 & 6,2 & 10,2 & 7,3 & 10,6 & 14,0 & 7,0 & 14,4 & 6,2 & 5,6 & 8,7 & \\
\hline 20,0 & 5,0 & 5,5 & 10,6 & 9,1 & 10,5 & 9,5 & 8,0 & 9,2 & 4,6 & 5,3 & 6,0 & \\
\hline 30,0 & 4,4 & 3,5 & 10,2 & 8,8 & 13,5 & 14,1 & 7,5 & 6,8 & 4,1 & 3,4 & 6,9 & \\
\hline 50,0 & 4,8 & 4,4 & 4,8 & 8,0 & 11,9 & 11,7 & 9,0 & 5,7 & 3,3 & 0,2 & 4,0 & \\
\hline 71,0 & 6,2 & 3,6 & 2,8 & - & 12,9 & 10,9 & 5,5 & 7,5 & 5,0 & 2,8 & 4,7 & \\
\hline
\end{tabular}

Das ausgeprägte Schichtungsbild während des Frühjahrs und der Sommermonate scheint uns ein direkter Beweis dafür $\mathrm{zu}$ sein, dass die Überführung von gelösten Phosphorverbindungen in PP zur Hauptsache durch photoautotrophe Organismen bewirkt wird und dass der grösste Teil des im See gefundenen PP in autochthon produzierter organischer Substanz fixiert ist.

\subsubsection{Gesamtphosphor}

Zur Zeit der Vollzirkulation werden das Phosphat $\left(\mathrm{PO}_{4}-\mathrm{P}\right)$, die gelösten organischen Phosphorverbindungen (GOP) und der geformte, partikuläre Phosphor (PP) annähernd homogen über die ganze Wassermasse verteilt. Nach dem Übergang in die stabile Schichtung werden der trophogenen Schicht die gelösten Phosphorverbindungen, namentlich das Phosphat, biogen entzogen und in PP verwandelt. Da aber das spezifische Gewicht der Phytoplankter nur in seltenen Fällen kleiner als oder gleich 1 ist, sinken im Süsswasser die meisten Organismen langsam ab. Es ist daher eine Verarmung der trophogenen Schicht an Gesamtphosphor während der Stagnationsperiode zu erwarten. Dabei ist zu berücksichtigen, dass, beschleunigt durch die relativ hohen Temperaturen, ein Teil des in absterbenden Organismen fixierten Phosphors bereits im Epilimnion autolytisch oder bakteriell freigesetzt wird und damit wieder 

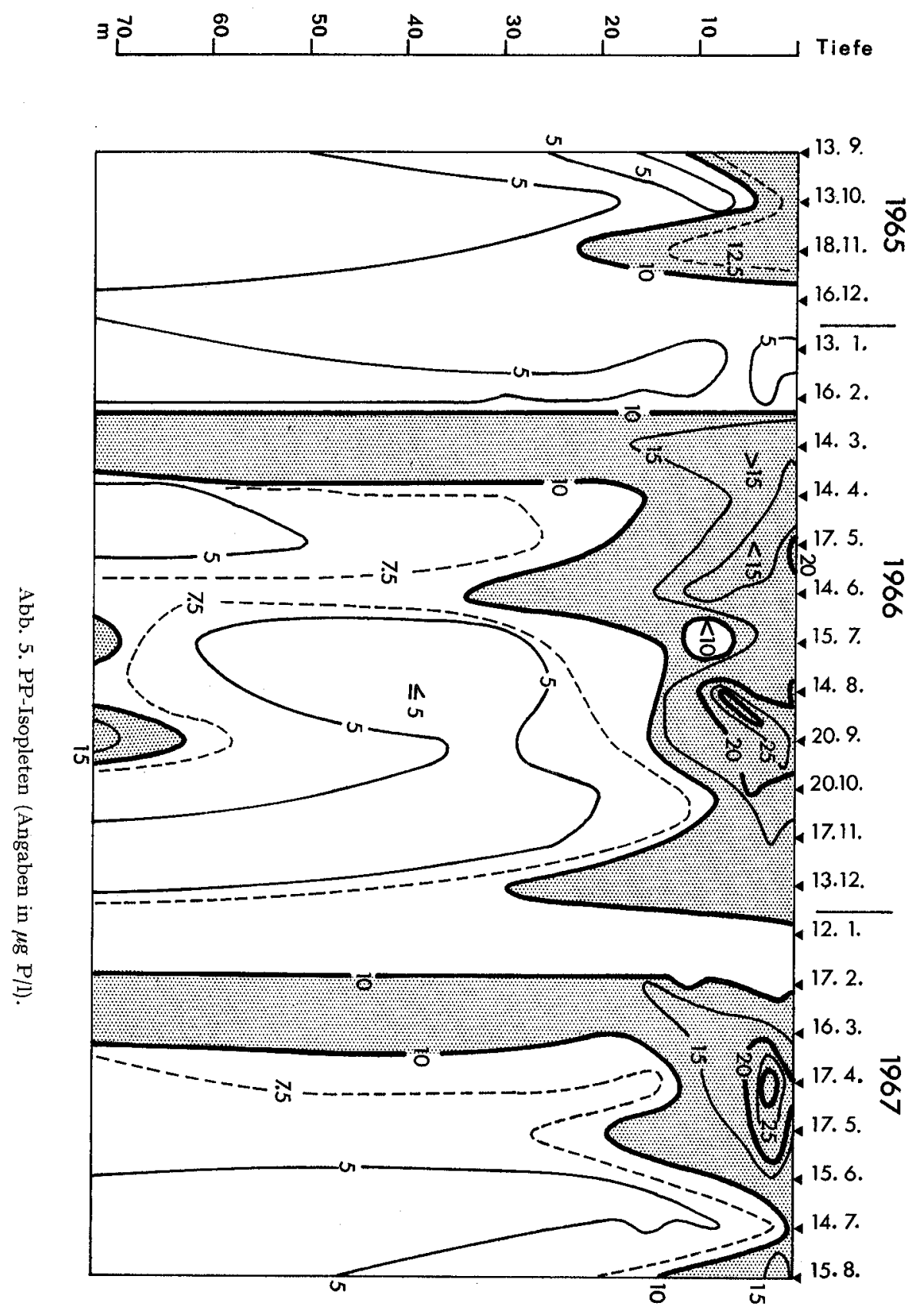
andern Organismen zur Verfügung steht. Dieser «kleine Kreislauf» arbeitet der Sedimentation von PP entgegen, vermag sie aber sicher auch im besten Fall nicht vollständig zu kompensieren. Leider ist über die Umsatzraten des Phosphors in diesem Kreislauf noch wenig bekannt, da die angewandte Untersuchungsmethode möglicherweise zu falschen Schlüssen führte [41].

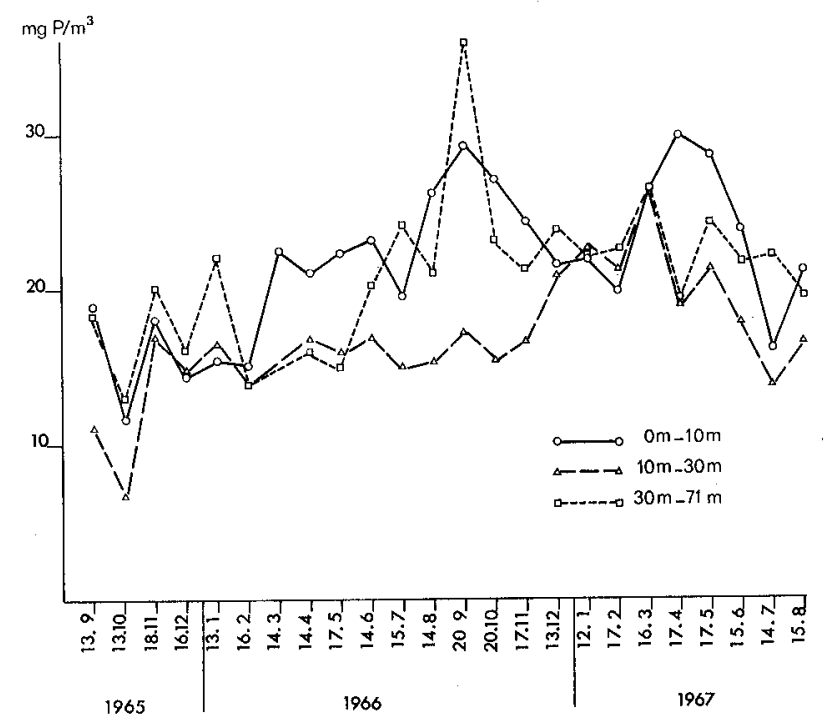

Abb. 6. Mittlere Gesamtphosphorkonzentrationen der drei Wasserschichten 0-10 m, 10-30 m und $30 \mathrm{~m}$ bis Grund.

In $\mathrm{Abb}, 6$ sind die mittleren Gesamtphosphorkonzentrationen der Wasserschichten 0-10 m, 10-30 m und $30 \mathrm{~m}$ bis Grund aufgetragen. Mitte Februar war der Gesamtphosphor in beiden Jahren mehr oder weniger homogen über die ganze Tiefe verteilt. Darnach nahm aber die Gesamtphosphorkonzentration der trophogenen Schicht nicht wie erwartet bis zum Einsetzen der Herbstzirkulation dauernd ab, sondern stieg bis zu einem ersten Maximum im Frühjahr an und erreichte nach einem Abfall der Konzentration im Sommer unmittelbar vor dem Einsetzen der Herbstzirkulation ein zweites Maximum. Durch die Herbstteilzirkulation wurde die Gesamtphosphorkonzentration der trophogenen Schicht nicht erhöht, sondern erniedrigt.

Die einzelnen Punkte der Kurve «30 m bis Grund» sind relativ schlecht gesichert, weil aus dieser Schicht jeweils nur drei Proben analysiert wurden und der Phosphorgehalt der untersten Probe $(71 \mathrm{~m})$ sehr stark von der genauen Höhe der Entnahmetiefe über dem Grund abhängt.

Die mittlere Gesamtphosphorkonzentration der Schicht «10-30 m» war während der Sommerstagnation immer kleiner als jene der obersten Wasserschicht und häufig niedriger als jene des Hypolimnions, so dass von einem eigentlichen "metalimnischen" Gesamtphosphorminimum gesprochen werden kann.

Wenn auch die einzelnen monatlichen Erhebungen nicht vorbehaltlos miteinander verglichen werden dürfen, da die entnommenen Proben kaum aus ein und demselben 
Wasserkörper stammen, so zeigt der generelle Verlauf der Kurve «0-10 m» doch, dass der Phosphorhaushalt dieses Sees nicht allein mit dem grossen Kreislauf (Zirkulation - Assimilation - Sedimentation - Dissimilation - Zirkulation) erklärt werden kann. Dass die trophogene Schicht trotz der Sedimentation von PP nicht an Gesamtphosphor verarmte, kann nur damit erklärt werden, dass ihr auch während der Stagnation dauernd Phosphorverbindungen zugeführt werden. Der momentane Gehalt der trophogenen Schicht an Gesamtphosphor während der Stagnationsperiode hängt also nicht nur von der Gesamtphosphorkonzentration während der Zirkulation (Basiswert, Grundlast) ab, sondern wesentlich von der Differenz zwischen ständiger Zufuhr und ständigem Verlust (Sedimentation und Abfluss). Da Basiswert, Zufuhr und Verlust zueinander sicher in einer bestimmten (wenn auch unbekannten) Beziehung stehen, mag der Basiswert allein zwar gewisse Schlüsse auf den Trophiegrad des Gewässers zulassen; es wäre aber vom produktionsbiologischen Standpunkt aus gesehen doch wünschenswert, diese Grössen getrennt bestimmen zu können.

BLösch (unveröffentlicht) hat versucht, die Phosphorzufuhr durch Messung der Sedimentation des PP in der Horwer Bucht indirekt zu bestimmen. $\mathrm{Zu}$ diesem $\mathrm{Zweck}$ hat er, wie Abb. 7 zeigt, das Sediment in verschiedenen Tiefen aufgefangen und die Sedimentation in Abschnitten von 14 Tagen gemessen.
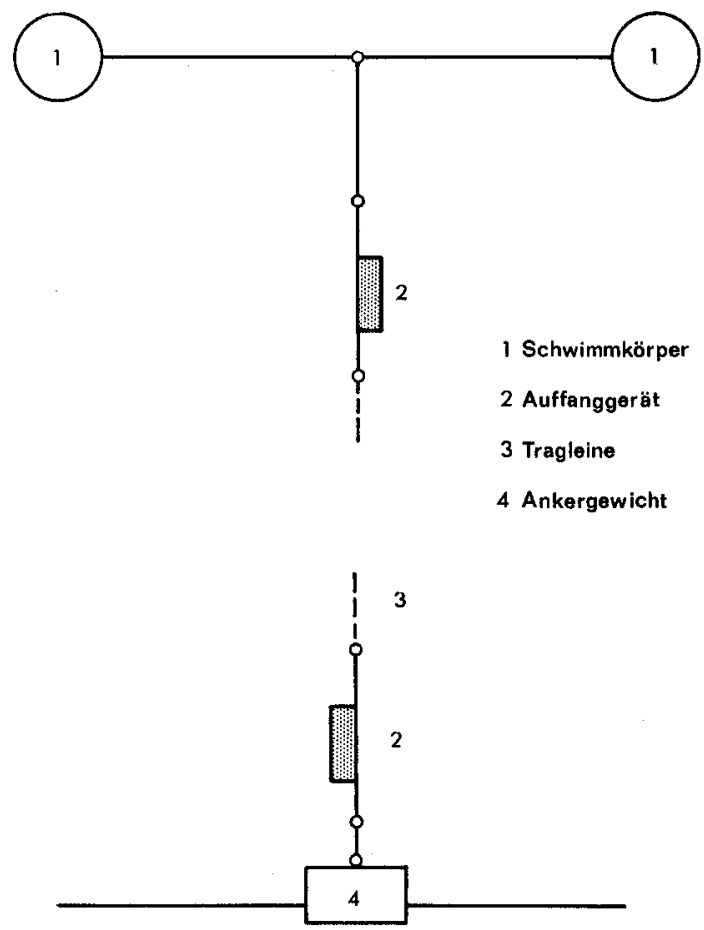

Abb. 7. Schematische Darstellung der Versuchseinrichtung zur Messung der Sedimentation des Sestons (nach BLÖSCH). 
Unter Vernachlässigung des Abflusses berechnet sich die mittlere minimale tägliche Phosphorzufuhr in einem bestimmten Zeitabschnitt wie folgt:

$$
Z=\text { Zufuhr in } \mathrm{mg} / \mathrm{m}^{3} \cdot \mathrm{Tag}=\frac{\left(C_{x}-C_{0}\right)+\mathrm{PP}_{\text {sed }} / V}{x},
$$

wobei $C_{\mathbf{0}}$ die mittlere Gesamtphosphorkonzentration des Wasservolumens $V$ zur Zeit null und $C_{x}$ jene zur Zeit $x$ bedeutet. Die Grösse $V$ gibt an, aus welchem Wasservolumen das Sediment während $x$ Tagen aufgefangen wurde. $\mathrm{PP}_{\text {sed. }}$ bezeichnet die während $x$ Tagen aufgefangene Menge PP.

Aus ersten Messungen liessen sich für die Wasserschicht $0-7,5 \mathrm{~m}$ die in Tab. 9 zusammengestellten Werte ermitteln. Dabei ist zu bedenken, dass die entnommenen Proben zur Zeit $t_{0}$ und $t_{x}$ höchstwahrscheinlich nicht aus ein und derselben Wassermasse stammen und daher $C_{0}$ und $C_{x}$ nur bedingt direkt miteinander verglichen werden dürfen. Dadurch kann der Wert $Z$ bei kurzer Versuchsdauer unter Umständen stark verfälscht werden. Die Grösse « $\mathrm{PP}_{\text {sed. }} \mathrm{mg} \mathrm{P} / \mathrm{m}^{\mathbf{3}} \cdot \mathrm{Tag}$, die angibt, wieviel «mg PP $/ \mathrm{m}^{3}$. Tag» der trophogenen Schicht verlorenging, scheint uns aber relativ gut gesichert zu sein.

Tab. 9.

\begin{tabular}{llll}
\hline Datum & $Z$ & $\begin{array}{l}\mathrm{PP} \text { sed. } \\
\mathrm{mg} \mathrm{P} / \mathrm{m}^{3} \text { pro Tag }\end{array}$ & $\begin{array}{l}\text { Täglicher Verlust } \\
\text { in \% PP }\end{array}$ \\
\hline 24. 8.- 6. 9.67 & 1,07 & 0,51 & 3,5 \\
$6.9 \cdot-20.9 .67$ & 0,08 & 0,47 & 3,1 \\
$20.9 \cdot-4 \cdot 10.67$ & 0,56 & 0,36 & 2,5 \\
$5.10 .-18.10 .67$ & 0,33 & 0,27 & 1,9 \\
\hline
\end{tabular}

Aus Tab. 9 geht hervor, dass die trophogene Schicht ohne ständige Zufuhr sehr rasch an Phosphor verarmen würde. Bei einem mittleren täglichen Verlust an PP von $2 \%$ berechnet sich die Halbwertzeit des PP auf 36 Tage, d.h., nach je 36 Tagen wäre die Konzentration der trophogenen Schicht an PP auf die Hälfte abgesunken.

Da dem Phosphor wachstumsbegrenzende Wirkung zugeschrieben wird, ist es klar, dass die jährliche Produktion des Gewässers nicht allein von der Ausgangskonzentration zur Zeit der Vollzirkulation abhängt, sondern wesentlich von der dauernden Zufuhr dieses Nährstoffs zur trophogenen Schicht beeinflusst wird. Daraus ergibt sich als theoretische Konsequenz zwangsläufig, dass bei totaler Drosselung der Phosphorzufuhr zur trophogenen Schicht während der Stagnationsperiode, unveränderte Biozönose vorausgesetzt, die Primärproduktion sofort merklich kleiner werden müsste. Weil es praktisch aber unmöglich ist, die Phosphorzufuhr zu einem Gewässer vollständig zu unterbinden, muss damit gerechnet werden, dass auch bei verringerter Belastung die jährliche Zufuhr noch grösser sein kann als die durch den Abfluss ausgeschwemmte und im Sediment irreversibel gebundene Menge. Aus diesem Grunde wird es in manchen Gewässern trotz getroffener abwassertechnischer Sanierungsmassnahmen unvermeidbar sein, dass sich der Basisphosphorgehalt weiter erhöht. $\mathrm{Ob}$ dieser Fall eintritt, hängt entscheidend davon ab, wieweit sich die Gesamtphosphorzufuhr zum Gewässer mit technischen Mitteln unterbinden lässt. Insofern hat die Frage nach dem prozentualen Anteil der abwassertechnisch erfass- 
baren Menge, verglichen mit der Gesamtbelastung, ihre Berechtigung. Wenn sich eine weitere Akkumulierung des Basiswerts aber auch nicht in jedem Falle verhindern lässt, so wird sie als Folge der Sanierungsmassnahmen immerhin verlangsamt voranschreiten. Es muss aber betont werden, dass selbst in diesen Fällen jedes Kilogramm Phosphat, das der trophogenen Schicht ferngehalten wird - die erwähnte wachstumsbegrenzende Funktion des Phosphors vorausgesetzt -, eine Verringerung der Primärproduktion bewirkt.

\subsubsection{Phosphorzufuhr zur trophogenen Schicht}

Es ist durchaus möglich, die Nährstoffzufuhr $z u$ einem Gewässer zu bestimmen. Auf beträchtliche Schwierigkeiten stösst man aber dann, wenn man über die horizontale und vertikale Ausbreitung dieser zugeführten Nährstoffe im Gewässer und damit über ihre momentan mögliche biologische Wirksamkeit Auskunft geben möchte. Noch komplizierter wird das Problem, wenn es $\mathrm{zu}$ berücksichtigen gilt, dass die Nährstoffzufuhr zur trophogenen Schicht auch während der Stagnation nicht nur von aussen (Abwasser, Zuflüsse, Niederschläge, Flugpollen, Blattfall, Wasservögel usw.), sondern auch von innen (Nährstoffregeneration, Zufuhr aus dem Hypolimnion und der Litoralzone) erfolgen kann.

Aufgenommene Nahrung wird von heterotrophen Organismen nur zum Teil in körpereigene Substanz verwandelt (assimiliert). Der Rest wird für den Energiestoffwechsel verwendet (veratmet) und als energieärmere chemische Verbindungen wieder aus dem Körper ausgeschieden. Daher leistet jeder Konsument (und unter ihnen namentlich das Zooplankton) einen Beitrag zur Regeneration von Pflanzennährstoffen [52-57].

Fraglich ist es aber - und das interessiert in diesem Zusammenhang besonders -, ob und in welchem Masse es durch seine täglichen vertikalen Wanderungen Phosphorverbindungen aus der tropholytischen in die trophogene Schicht transportiert und damit der Sedimentation von PP entgegenwirkt.

Besonders dann, wenn die trophogene Schicht mit dem Epilimnion nicht identisch ist, muss damit gerechnet werden, dass durch Diffusion und Turbulenz Nährstoffe in die trophogene Schicht gelangen (vgl. S. 16).

Litoral und Pelagial beeinflussen sich durch horizontale Strömungen gegenseitig [58]. Weil während des Sommers im Litoralbereich das Wasser und vor allem das Sediment wärmer ist als im Pelagial, ist zu erwarten, dass die Mineralisationsvorgänge rascher und vollständiger verlaufen als im offenen Wasser. Eine dadurch bedingte Nährstoffzufuhr zum pelagischen Epilimnion müsste unter sonst identischen Verhältnissen um so stärker ins Gewicht fallen, je flacher der Seegrund am Ufer abfällt und je grösser das Flächenverhältnis von Litoralzone zu Profundalzone ist. Leider ist es uns nicht möglich, diese Phosphorzufuhr zur trophogenen Schicht aus den beiden «Depots» Hypolimnion und Litoral auch nur näherungsweise zu schätzen. Vergleicht man aber mit der allochthonen Belastung, so darf angenommen werden, dass sie wahrscheinlich vernachlässigt werden kann.

In die Horwer Bucht gelangt durch einen kleinen Kanal, oberflächlich eingeleitet, das Abwasser von rund 10000 Einwohnern. WuHrMann [59] rechnet mit einem Basiswert von $3 \mathrm{~g} P$ pro Kopf und Tag in ungeklärtem Abwasser. Da die meisten Häuser Hausklärgruben besitzen, dürfte dieser Wert für unsere Verhältnisse etwas zu hoch sein. Er wird aber auch bei vorsichtigster Schätzung 2 g P pro Kopf und Tag 
nicht unterschreiten. Daraus berechnet sich die minimale jährliche Belastung bei der Annahme von rund $2 \mathrm{~km}^{2}$ Seefläche auf $3,6 \mathrm{~g} / \mathrm{m}^{2}$. Jahr. (In dieser Berechnung wurde der Anteil der gewerblichen und industriellen Abwässer nicht berücksichtigt).

Das direkte Einzugsgebiet umfasst rund $16 \mathrm{~km}^{2}$ hauptsächlich forstwirtschaftlich genutztes Gebiet. Setzt man nach АмвӥнL [60] für Auswaschung und oberflächliche Abschwemmung 0,3-0,5 kg P/ha. Jahr ein, so werden der Bucht auf diese Weise jährlich 480-800 kg Phosphor zugeführt. Das entspricht 6,5-12\% der durch Abwasser zugeführten Menge. Da sich die Angaben von АмвüHL [60] auf hauptsächlich landwirtschaftlich genutztes Gebiet beziehen, dürften diese Werte in Wirklichkeit geringer ausfallen.

Eine Berechnung der Belastung unter der Annahme, dass das Abwasser der Gemeinde Horw nur die Horwer Bucht beeinflusse, ist freilich problematisch. Da aber die angrenzenden Seeteile ihrerseits ebenfalls belastet werden (Abwasser von Hergiswil), dürfte der erhaltene Wert zumindest in der richtigen Grössenordnung liegen.

Es darf als sicher angenommen werden, dass sich das zugeführte Abwasser während der Sommerstagnation zum grössten Teil in die trophogene Schicht $(0-10 \mathrm{~m})$ einschichtet. Bei homogener Verteilung innerhalb dieser Schicht würde sich dabei eine tägliche $Z$ ufuhr von $1 \mu \mathrm{g} P / 1$ ergeben. Interessanterweise ist dieser Wert höher als der von BlOESCH ermittelte tägliche Sedimentationsverlust (vgl. S. 23). Es geht daraus hervor, dass bereits der Abwasseranteil ausreicht, um die tägliche Sedimentation von $\mathrm{PP}$ zu kompensieren und damit die erwünschte natürliche Verarmung der trophogenen Schicht während der Stagnationsperiode zu verhindern.

\subsection{Biologische Untersuchungen}

\subsubsection{Qualitative Phytoplanktonanalyse}

Das Phytoplankton wurde von U. ZimmermanN monatlich im umgekehrten Mikroskop ausgezählt. Auf diese Resultate soll hier nur soweit eingegangen werden, als es zur Beantwortung verschiedener Teilfragen nötig erscheint. Mit der räumlichen und zeitlichen Verteilung der einzelnen Formen, namentlich von Oscillatoria rubescens, wird sich ZIMmermann [66] eingehender auseinandersetzen.

In Tab. 10 sind die Individuen, die in der trophogenen Schicht $(0-10 \mathrm{~m})$ unter $1 \mathrm{~m}^{2}$ Seefläche gefunden wurden, zusammengestellt. Arten, welche in so geringer Zahl auftraten, dass sie nicht statistisch gesichert ausgezählt werden konnten, sind mit + bezeichnet. Wird neben der Anzahl auch noch das Volumen der einzelnen Arten berücksichtigt, so ist daraus ersichtlich, dass Oscillatoria rubescens während des ganzen Jahres einen wesentlichen Anteil an der Gesamtbiomasse ausmachte.

Als zweitwichtigste Form ist während eines Grossteils des Jahres Tabellaria fenestrata zu nennen. Im Sommer müssen ferner Ulothrix amphigranulata, im Frühjahr Stephanodiscus hantzschii, Rhodomonas spp. (Rh. lacustris + Rh. lens) und Cryptomonas spp. (Cr. ovata $+C r$. erosa) berücksichtigt werden.

In Tab. 11 sind die unter $1 \mathrm{~m}^{2}$ Seefläche gefundenen Biomassen zusammengestellt. Unter der Annehme, dass der Kohlenstoffgehalt des Phytoplanktons 10\% des Frischgewichts betrage, lässt sich der tägliche Erneuerungskoeffizient $\mathrm{zu}$

$$
\frac{10 \cdot C_{\text {ass }} / \mathrm{m}^{2} \cdot \mathrm{Tag}}{\text { Biomasse } / \mathrm{m}^{2}}
$$




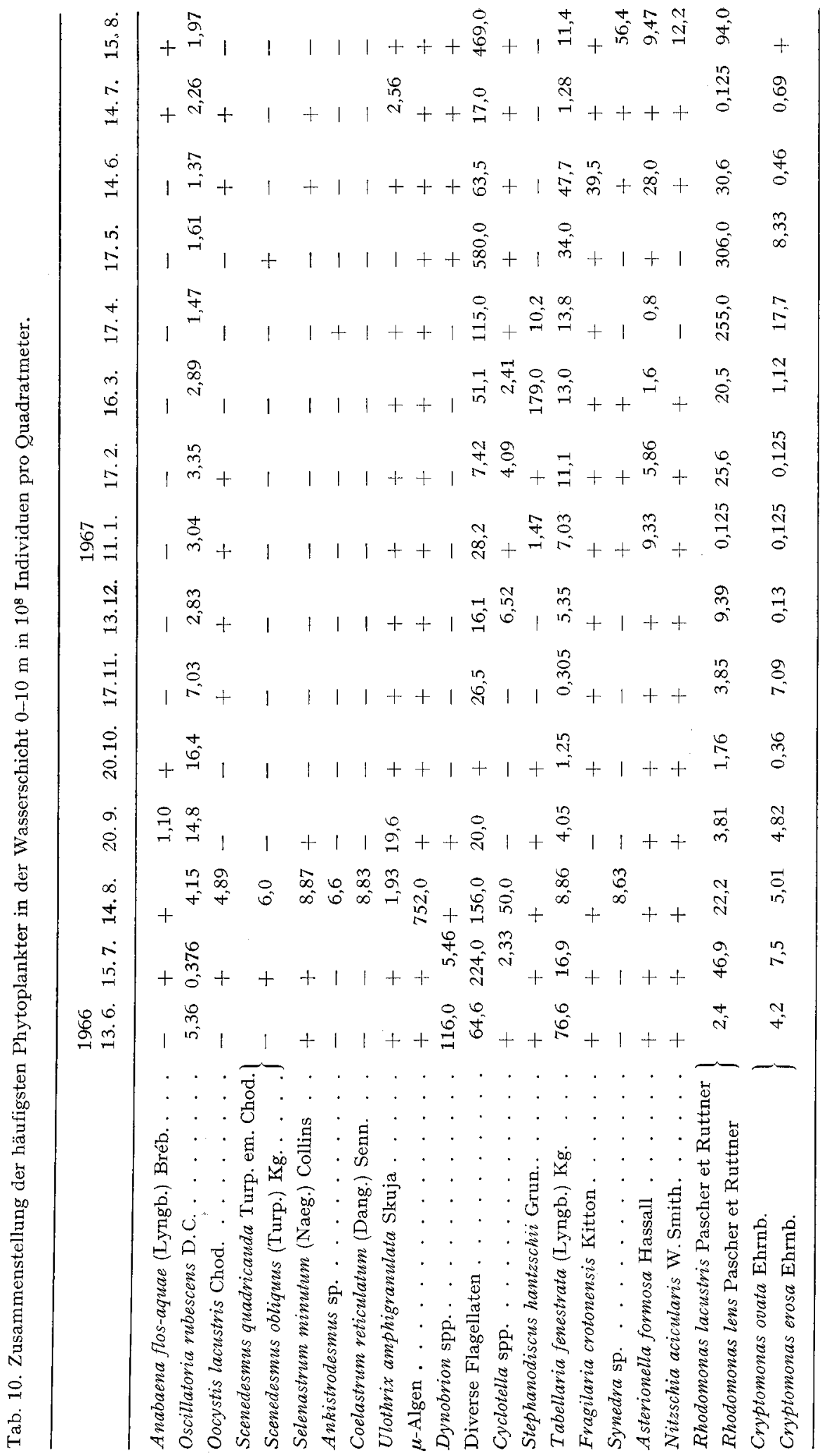


Tab. 11 .

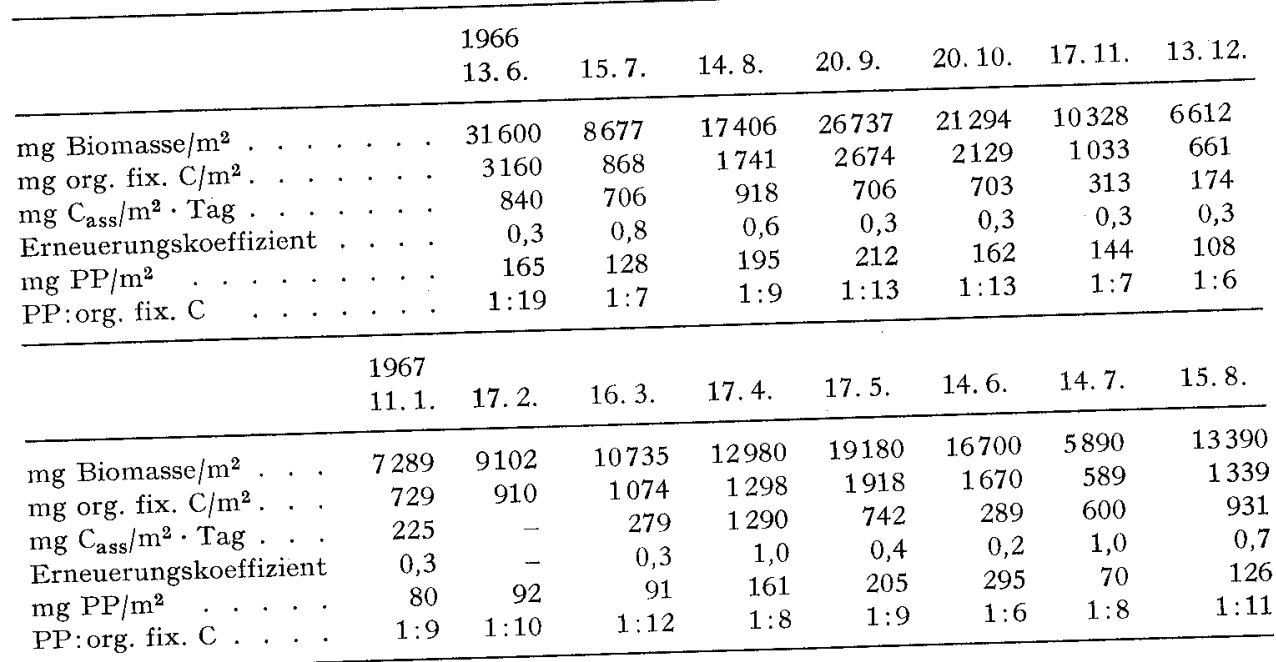

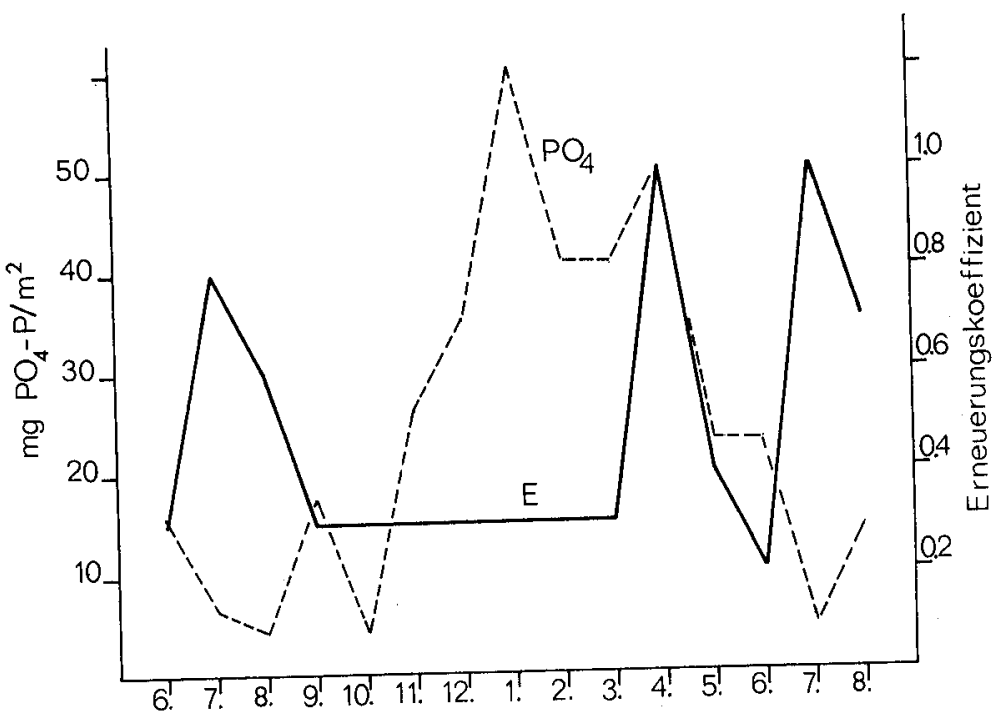

Abb. 8. Erneuerungskoeffizient und mittlerer Phosphatgehalt der Wasserschicht 0-10 $\mathrm{m}$.

berechnen [67]. Er muss als Mass für die Aktivität, von der qualitativen Zusammensetzung des Phytoplanktons abgesehen, um so grösser sein, je besser die Wachstumsverhältnisse sind. In Abb. 8 wird der Erneuerungskoeffizient der in der trophogenen Schicht unter $1 \mathrm{~m}^{2}$ Seefläche gefundenen Menge $\mathrm{PO}_{4}-\mathrm{P}$ gegenübergestellt. Es geht daraus deutlich hervor, $C$. $2 s s$ die höchste Phosphatkonzentration keineswegs mit einer hohen Aktivität des Planktons zusammenfällt. Es scheint umgekehrt vielmehr so zu sein, dass eine hohe Aktivität des Phytoplanktons die Phosphatkonzentration in der trophogenen Schicht so weit erniedrigt, dass sie nachher produktionsbegrenzend wird. 
Wir gelangen so auf einem andern Weg zum selben Resultat wie GRIM [17], nämlich, dass während eines Teils des Jahres klimatische Faktoren produktionsbegrenzend sind.

\subsubsection{Primärproduktion und Phosphor}

Unter der Primärproduktion versteht man die primäre Produktion von organischen Verbindungen durch autotrophe Organismen. Die dazu benötigte Energie liefert das Sonnenlicht (Photosynthese) oder die oxydative Verarbeitung von anorganischen Substraten (Chemosynthese). Die Kohlenstoffassimilation schafft nicht nur wichtige Bausteine, sondern bindet in den Kohlehydraten auch die $\mathrm{zu}$ weitern Synthesen (z.B. Eiweißsynthese) benötigte Energie. Sie ist daher ein Mass für den Energiegewinn des synthetisierenden Systems (z.B. Phytoplankton), und damit für die Primärproduktion.

Die Photosyntheseleistung des Phytoplanktons wurde monatlich mit der $\mathrm{C}^{\mathbf{1 4}}$ Technik in situ gemessen. Die Proben wurden während 4 Stunden von 10 bis 14 Uhr exponiert. In diesem Zeitabschnitt wird unter $1 \mathrm{~m}^{2}$ Seefläche etwa $30-50 \%$ der täglich assimilierten Kohlenstoffmenge aufgenommen. Die Resultate der einzelnen Messungen sind in Tab. 12 zusammengestellt.

REDFIELD et. al. [63] geben als mittleres Atomverhältnis für Plankton $\mathrm{P}: \mathrm{C}=$ 1:106 an. Dies entspricht einem Gewichtsverhältnis von 1:40. Umgerechnet nach Angaben von AMBÜHL [60] ergibt sich für das Phytoplankton im Vierwaldstättersee ein mittlerer Phosphorgehalt von rund 0,5\%, bezogen auf das Frischgewicht (Biomasse berechnet nach LoHMANN [62]). Rechnet man nach NAuwERCK [67] mit 10\% organisch gebundenem Kohlenstoff (ebenfalls auf das Frischgewicht bezogen), so ergibt sich daraus ein $P: C$-Verhältnis von rund $1: 20$, nach eigenen Untersuchungen ein solches von etwa 1:10 (s. Tab. 11). Dabei muss aber berücksichtigt werden, dass ein Teil des bestimmten PP nicht organismisch fixiert, sondern mit Detritus assoziiert ist. Die Angaben von REDFIELD [63] dürften deshalb zumindest näherungsweise auch für das Plankton im Vierwaldstättersee gelten.

Aus dem bekannten Verhältnis P:C (1:40) und der gemessenen Kohlenstoffassimilation lässt sich die Phosphoraufnahme des Phytoplanktons in der trophogenen Schicht abschätzen. In Tab. 13 sind die berechneten mittleren täglichen Aufnahmeraten den aktuellen Konzentrationen gegenübergestellt. Diese Gegenüberstellung

Tab. 12. Photosyntheseraten (Angabe in $\mathrm{mg} C_{\text {ass }} / \mathrm{m}^{3} \cdot \mathrm{h}$ ).

\begin{tabular}{|c|c|c|c|c|c|c|c|c|c|c|c|c|c|c|}
\hline $\begin{array}{l}\text { Tiefe } \\
{[\mathrm{m}]}\end{array}$ & $\begin{array}{l}1966 \\
13.6 .\end{array}$ & 15.7. & 14.8. & 20.9 & & & & $\begin{array}{l}1967 \\
11.1 .\end{array}$ & 16.3. & 17. 4.1 & 17.5. & 14.6. & 14.7. & 15. \\
\hline 0,0 & 17,90 & 15,40 & 7,40 & 5,00 & 3,71 & 4,25 & 1,18 & 0,82 & 1,80 & 95,00 & 7,04 & 1,54 & 37,00 & \\
\hline 1,0 & - & 16,10 & 16,60 & 11,70 & 10,20 & 4,60 & 1,59 & 1,28 & 2,38 & 41,001 & 15,70 & 8,20 & 16,3 & 20 \\
\hline 2,5 & 12,31 & 14,30 & 18,40 & 13,80 & 17,00 & 4,73 & 2,22 & 2,0 & 3,54 & 12,90 & 15,65 & 5,10 & 4,96 & 16,2 \\
\hline 5,0 & 6,04 & 3,40 & 5,60 & 7,80 & 7,95 & 3,55 & 1,49 & 2,51 & 3,73 & 1,96 & 6,60 & 2,90 & 1,66 & 5,7 \\
\hline 7,5 & 2,75 & 1,06 & 5,10 & 1,48 & 0,64 & 1,39 & 1,17 & 2,36 & 2,26 & 1,96 & 1,36 & 0,33 & 1,05 & 2,6 \\
\hline 10,0 & 0,95 & 0,80 & 0,85 & 0,32 & 0,21 & 0,51 & 0,95 & 1,72 & 0,74 & 26,70 & 0,35 & 0,12 & 0,86 & 2,2 \\
\hline 12,5 & 0,21 & 0,00 & 0,00 & 0,00 & 0,00 & 0,30 & 0,42 & - & 0,41 & 3,65 & 0,00 & 0,00 & 1,14 & 2,17 \\
\hline 15,0 & 0,00 & - & - & - & - & 0,00 & 0,00 & - & - & - & - & - & 0,16 & 0,44 \\
\hline $\mathrm{mg} C_{a s s} / \mathrm{m}^{2} \cdot \mathrm{h}$ & 83,9 & 70,6 & 91,8 & 70,6 & 70,3 & 31,3 & 17,4 & 22,5 & 27,9 & 129,0 & 74,2 & 28,9 & 60,0 & 93,1 \\
\hline
\end{tabular}




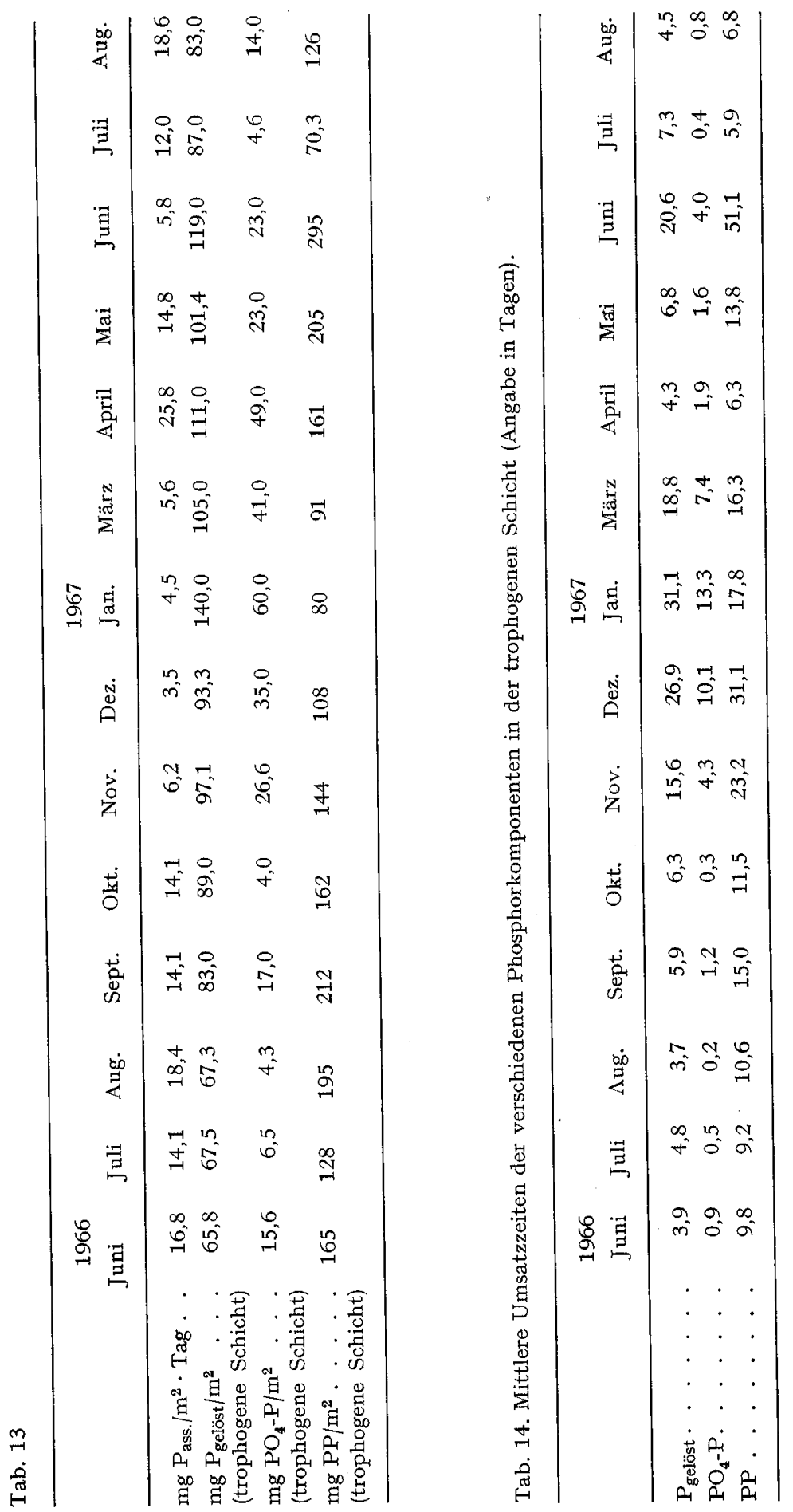


zeigt deutlich, dass die umgesetzte Menge Phosphor zumindest nicht allein von der aktuell vorhandenen Phosphatkonzentration abhängt. Vielmehr ist es so, dass bei höchstem Nährstoffangebot im Winter, wahrscheinlich bedingt durch die niedrige Temperatur und die schwache Einstrahlung, pro Zeiteinheit die geringste Phosphormenge «assimiliert» wird.

Durch die Aufnahmerate und die aktuelle als konstant angenommene Konzentration ist die Umsatzzeit $t$ gegeben (Tab. 14).

$$
t=\frac{\text { aktuell vorhandene Nährstoffmenge }}{\text { aufgenommene Nährstoffmenge/Zeit }}
$$

(Wird die trophogene Schicht als geschlossenes System betrachtet, so entspricht die "aufgenommene Nährstoffmenge pro Zeit» der Umsatzrate des kleinen Kreislaufs.)

Sie gibt an, in welcher Zeitspanne z.B. die aktuelle Phosphatkonzentration einmal umgesetzt wird. Die Umsatzzeiten des Phosphats sind im Sommer gemäss Tab. 14 rund eine Zehnerpotenz niedriger als im Winter. Interessant ist in diesem Zusammenhang ein Vergleich von täglich zugeführter und tägliche umgesetzter Phosphormenge im Sommer:

A. Mittlere Aufnahmerate in der

trophogenen Schicht

$15 \mathrm{mg} \mathrm{P} / \mathrm{m}^{2}$. Tag (s. Tab. 13)

B. Mittlere Zufuhr zu dieser Schicht $5 \mathrm{mg} \mathrm{P} / \mathrm{m}^{2}$. Tag (s. Tab. 9)

C. Durch kleinen Kreislauf geliefert $10 \mathrm{mg} \mathrm{P} / \mathrm{m}^{2} \cdot \mathrm{Tag}$ (A.-B.)

Vergleicht man die Umsatzrate des kleinen Kreislaufs (C.) mit der gesamten Menge PP $/ \mathrm{m}^{2}$ in der trophogenen Schicht (Tab. 13), so sieht man, dass täglich $5-10 \%$ des PP freigesetzt und organismisch wieder fixiert werden. Ein Teil dieses freigesetzten Phosphors stammt aus absterbenden oder abgestorbenen Organismen. GOLTERMANN [64] hat gezeigt, dass P-Verbindungen autolytisch wesentlich rascher freigesetzt werden als N-Verbindungen. Es ist anzunehmen, dass auf diese Weise bereits in der trophogenen Schicht freigesetzter Phosphor die Produktion von neuer organischer Substanz ermöglicht, während der absinkende Detritus, der zwar an Phosphor verarmt, aber doch nicht vollständig mineralisiert ist, zur hypolimnischen Sauerstoffzehrung beiträgt. Dadurch kann die Belastung eines Gewässers mit autochthon produzierter organischer Substanz um ein Vielfaches grösser sein, als es aufgrund der aktuellen epilimnischen Phosphatkonzentration erwartet werden könnte.

Sicher sind die Umsatzraten des PP, wenn alle andern Bedingungen gegeben sind, auch von der aktuellen PP-Konzentration abhängig. Daher muss sich, wenn Phosphor begrenzender Faktor ist, eine erhöhte Phosphatzufuhr zur trophogenen Schicht in doppelter Weise auswirken:

erstens als momentan zur Verfügung stehendes Nährsalz und zweitens durch die Erhöhung der PP-Konzentration und damit der Umsatzrate.

\subsubsection{Primärproduktion und Licht}

In optisch homogenem Wasser nimmt die Intensität von monochromatischem Licht exponentiell zur passierten Schichtdicke ab. Diejenige Schichtdicke, in der die Intensität auf die Hälfte absinkt, wird als Einheit der optischen Tiefe definiert. (In der optischen Tiefe 1 werden also noch $50 \%$, in der optischen Tiefe 2 noch $25 \%$ der 
Oberflächenenergie registriert usw.) Vergleicht man im Experiment die Photosyntheseraten mit der gebotenen Lichtintensität, so sieht man, dass sie im untern Bereich $\left(0-0,5 I_{K}\right)$ proportional der steigenden Lichtintensität zunehmen. Bei weiterer Steigerung der Lichtintensität erfolgt eine immer geringere Zunahme der Assimilationsintensität, bis sie ein Optimum erreicht $\left(A_{\text {max }}\right)$, auf diesem verharrt und bei noch weiterer Steigerung der Lichtintensität wieder absinkt.

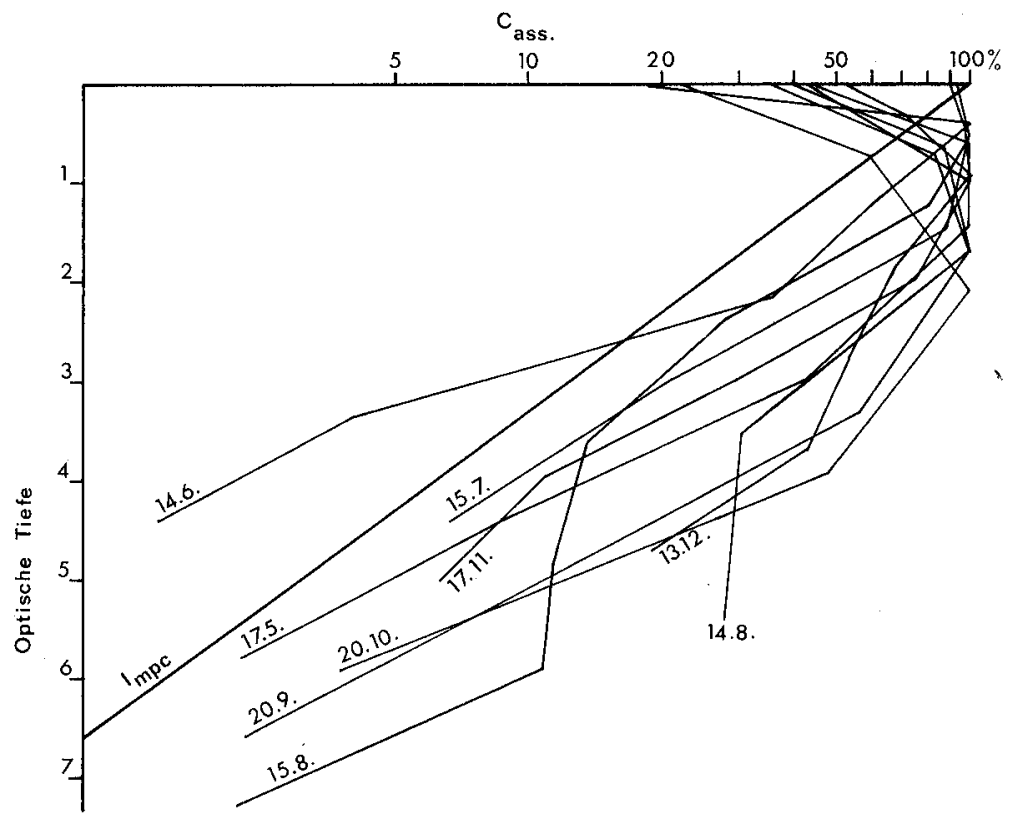

Abb. 9. Assimilationskurven im halblogarithmischen Maßstab gegen die optische Tiefe aufgetragen.

Trägt man die prozentualen Assimilationsraten, bezogen auf $A_{\max }=100 \%$, im halblogarithmischen Maßstab gegen die Tiefe auf, so ist nach RoDHE [65] unterhalb der optischen Tiefe $3\left(0,5 I_{K}\right)$ ein paralleler Verlauf der Photosynthesekurve zum Intensitätsabfall derjenigen Lichtkomponente $z u$ erwarten, die den kleinsten vertikalen Extinktionskoeffizienten aufweist. Setzt man für die Respiration $10 \%$ von $A_{\text {max }}$ ein, und nimmt an, dass in der optischen Tiefe 3 pro Zeiteinheit $74 \%$ von $A_{\max }$ assimiliert wird, so würde der Kompensationspunkt ungefähr mit jener Tiefe zusammenfallen, in welcher die Intensität der Lichtkomponente mit dem kleinsten vertikalen Extinktionskoeffizienten $\left(I_{m p c}\right)$ auf $1 \%$ der Oberflächenintensität abgesunken ist. Daher sollte es möglich sein, die Ausdehnung der trophogenen Schicht aufgrund des vertikalen Extinktionskoeffizienten von $I_{m p c}$ abzuschätzen.

Wie aus Tab. 2 hervorgeht, dringt in der Horwer Bucht das grüne Licht (VG9) ausnahmslos am tiefsten ein. In Tab. 15 sind jene Tiefen zusammengestellt, in welchen noch $1 \%$ der an der Oberfläche gemessenen Intensität $I_{0,05}$ gemessen wurde.

In $\mathrm{Abb} .9$ sind die relativen Photosyntheseraten (nach RoDHe [65]) im halblogarithmischen Maßstab gegen die Tiefe aufgetragen. Dabei wurden nur «Schön- 
Tab. 15. Tiefen, in welchen die Intensität des grünen Lichtes auf $1 \%$ der Oberflächenintensität $\left(I_{0,05}\right)$ abgesunken war (Angaben in $\mathrm{m}$ ).

\begin{tabular}{rllllllllllllll}
\hline 1966 & & \multicolumn{1}{c}{1967} & & & & \\
13.6. & 15.7. & 14.8. & 20.9 & 20.10. & 17.11. & 13.12. & 11.1. & 17.2. & 17.4 & 17.5. & 14.6. & 15.8. \\
\hline $0,01 I_{\mathbf{0}, 05}$ & 9,8 & 12,5 & 8,5 & 9,7 & 13,3 & 18,0 & 18,4 & 21,2 & 20,2 & 24,5 & 13,8 & 17,5 & 13,8 \\
\hline
\end{tabular}

wetterkurven", d.h. Kurven, die in den obersten Schichten eine Hemmung der Photosynthese zeigen, berücksichtigt. Im allgemeinen herrschte gute Übereinstimmung zum Photosynthesemodell von RoDHE. In tieferen Schichten nahmen die Photosyntheseraten jedoch in der Regel etwas rascher ab, als es aus dem Verlauf der Abnahme des grünen Lichtes zu erwarten wäre. Dadurch wird die Grenze der positiven Assimilationsbilanz etwas angehoben. Sie fiel in den meisten Fällen in jenen Bereich, in welchem noch $1-5 \%$ von $I_{0,05}$ gemessen wurde. Das bedeutet, dass die Primärproduktion vom Frühjahr bis zum Herbst im wesentlichen auf die obersten $10 \mathrm{~m}$ beschränkt blieb.

Am 14. August 1966 und am 15. August 1967 wich der Verlauf der Assimilationskurven deutlich vom "Normalverlauf» ab. Unterhalb der optischen Tiefe 3,5 (das entspricht 5 bzw. 7,5 m) fielen die Photosyntheseraten plötzlich langsamer ab, als es aus der Abnahme der Lichtintensität erwartet wurde. Diese "Abnormität» war auf eine stark inhomogene Verteilung des Phytoplanktons (Einschichtung von Oscillatoria rubescens) zurückzuführen. Im Mittel fielen die Photosynthesekurven unterhalb der optischen Tiefe 2,5 ungefähr parallel zu $I_{m p c}$ ab, und die mittlere Assimilationsrate bei der Lichtintensität $0,5 I_{K}$ betrug etwa $70 \%$ des maximal beobachteten Wertes.

\section{Versuche}

Von wenigen Ausnahmen abgesehen, worunter namentlich polytrophe Gewässer fallen [11], tritt Orthophosphat in der trophogenen Schicht von Seen entweder schon kurz nach Beginn oder im Laufe der Stagnationsperiode nur in sehr niedrigen Konzentrationen auf. Diese Beobachtung hat zum Schluss geführt, dass das Phosphat in dieser Zeit infolge seines Fehlens das weitere Wachstum des Phytoplanktons begrenze. Angesichts der spärlichen experimentellen Grundlagen, insbesondere im halbtechnischen oder technischen Maßstab, welche den vorhandenen Resultaten aus Laboruntersuchungen erhöhte Sicherheit verleihen könnten, haben wir uns im Rahmen dieser Arbeit die Aufgabe gestellt, die Wirkung erhöhter Phosphorkonzentrationen auf das natürliche Seeplankton möglichst unter Konstanthaltung aller übrigen ökologischen Komponenten (in situ) zu prüfen.

Beantwortet das Phytoplankton eine Phosphatdüngung mit erhöhter Primärproduktion, so kann daraus geschlossen werden, dass die Wirkung dieses Faktors gegen das Optimum hin verschoben wurde, dass er also unter den ursprünglichen natürlichen Bedingungen begrenzend wirkte.

Um die Wirkung von zugesetztem Phosphat unter möglichst natürlichen Bedingungen studieren zu können, wurden an die zu konstruierende Versuchseinrichtung die folgenden Forderungen gestellt: 
- Um den Effekt der künstlichen Substratfläche (= innere Oberfläche der Kulturgefässe) auf ein Minimum herabzusetzen und um die Konzentration des zugesetzten Nährsalzes in realistischen Grenzen halten zu können, sind die Kulturtanks so zu bemessen, dass das Verhältnis von Oberfläche zu Volumen klein wird.

- Um das Plankton in der Schwebe halten zu können, muss in den Kulturtanks eine geringe Turbulenz erzeugt werden können.

- Das Material, aus dem die Kulturtanks gebaut werden, muss biologisch inert sein.

- Es muss transparent sein und darf zumindest im sichtbaren Bereich das Licht nicht selektiv absorbieren. (IR und UV sind in der zu exponierenden Tiefe z.T. bereits durch das Wasser absorbiert.)

- Es muss gegenüber Ionen einen sehr hohen Diffusionswiderstand aufweisen und darf sie nur in geringem Masse adsorbieren.

- Schliesslich soll es eine elastische Bauweise ermöglichen, damit trotz der Entnahme von Proben im Innern des Tanks kein Unterdruck entsteht.

Diese Bedingungen wurden von $100 \mu \mathrm{m}$ dicker Plastikfolie (Polyäthylen) gut erfüllt. Da solch dünne Folie aber den mechanischen Beanspruchungen im Experiment nicht gewachsen war, musste eine Konstruktion gefunden werden, die es ermöglichte, dass dieser feine, hermetisch abschliessende Plastikzylinder auch bei starkem Wellengang mechanisch nicht beansprucht wurde. Abb. 10 zeigt schematisch die Konstruktion der "Nährstofftestzellen» (NTZ). Der innere Plastikzylinder von $100 \mu \mathrm{m}$ Wandstärke hängt nicht frei im Wasser, sondern schwebt in einem starren, transparenten, mit Wasser gefüllten Zylinder, der kleine Kommunikationen zum See aufweist, damit während der Probenahme der Druckausgleich möglich ist. Er ist aus verzinktem Armierungsnetz von $15 \mathrm{~cm}$ Maschenweite gefertigt, mit starker Plastikfolie ausgekleidet und an drei Bojen aufgehängt. Durch die beiden Folien werden etwa $10 \%$ des Lichtes absorbiert. Das Kulturvolumen beträgt rund $1400 \mathrm{1}$. Die nötige Turbulenz wird durch ein kleines Rührtellerchen erzeugt, das direkt mit einem Schwimmer verbunden ist. Es ist anzunehmen, dass bei dieser direkten Úbertragung von mechanischer Energie von der Oberfläche in die Tiefe, die so erzeugte Turbulenz die natürliche übertrifft. Rührteller, Rührstange und Verschlussarmatur sind aus Hart-PVC gefertigt. Die Durchführung des Rührgestänges ins Zelleninnere wurde mit einem leicht beweglichen Faltenbalg aus Gummi abgedichtet.

Die Aufhängevorrichtungen sind so bemessen, dass die NTZ im Bereich jener Tiefe exponiert sind, in welchem im allgemeinen maximale Assimilationsraten gemessen werden (mittlere Tiefe $=2,5 \mathrm{~m}$ ). Aus dieser Tiefe wurde auch das Seewasser entnommen und mit einer handbetriebenen Kolbenpumpe möglichst schonend in die Zellen gefüllt. Eine NTZ enthielt als Vergleichsprobe unverändertes Seewasser. In den andern wurde die Phosphorkonzentration durch $\mathrm{Zusatz}$ von $\mathrm{NaH}_{2} \mathrm{PO}_{4} \cdot 2 \mathrm{H}_{2} \mathrm{O}$ (Merck) erhöht. Bereits im ersten Vorversuch wurde festgestellt, dass der unerwünschte Algenaufwuchs auf den Plastikfolien und vor allem der sich auf dem Deckel der NTZ ansammelnde biogen gefällte Kalk den Lichtgenuss der Planktonkulturen stark beeinträchtigt hätte. Die NTZ wurden daher durch Taucher regelmässig kontrolliert und gereinigt.

Zur Entnahme von Proben wurde durch den «Probenahmeschlauch» ein zweiter dünner Schlauch eingeführt, durch welchen das Wasser mit Hilfe einer Saugpumpe aus jeder beliebigen Höhe unmittelbar in die Probeflasche gesaugt werden konnte. 


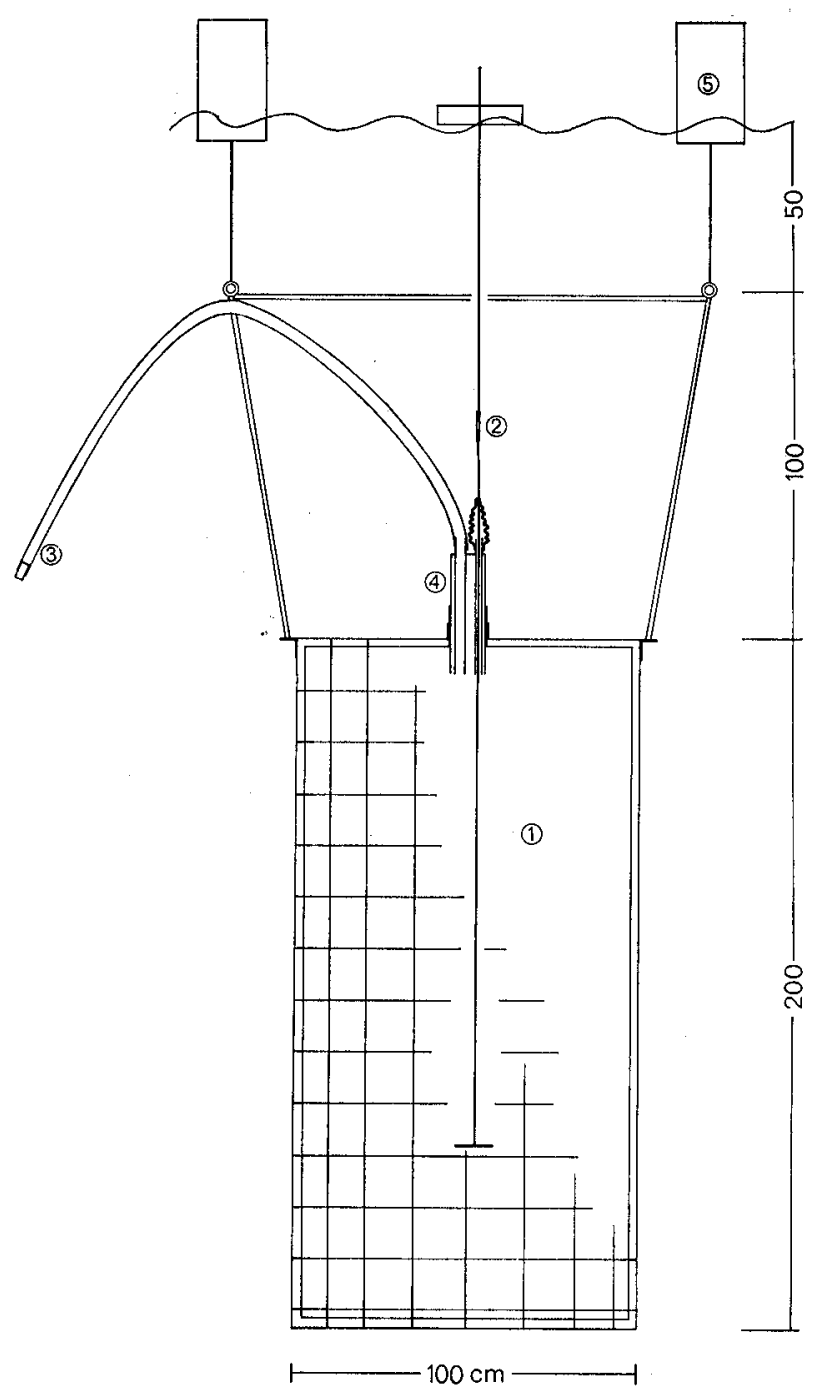

Abb. 10. Schematische Darstellung der Nährstofftestzellen. 1 Plastikzylinder, 2 Rührstange mit Schwimmer und Rührteller, 3 Probenahmeschlauch, 4 Verschlussarmatur, 5 Boje.

Durch diesen zweiten Schlauch konnten der Kultur auch jederzeit Nährstoffe zugeführt werden, ohne dass die Nährstofftestzelle an die Oberfläche gehoben werden musste. Um möglichst repräsentative Proben zu erhalten, wurden aus sechs verschiedenen Tiefen der Zelle Wasser entnommen und daraus eine Mischprobe hergestellt. Die Probenahmen wurden zeitlich so angesetzt, dass sie unmittelbar vor dem lokalen Sonnenaufgang beendigt waren. Dadurch wurden die Proben immer zur gleichen "physiologischen Zeit» entnommen, und man hatte die Gewähr, dass das Phytoplankton nicht durch den Einfluss von direktem Sonnenlicht geschädigt wurde. 
Ein Teil der so gewonnenen Mischprobe wurde mit $\mathrm{C}^{14}$ versetzt und im Rotationslichtthermostaten während 4 Stunden exponiert. Dabei wurde die Temperatur während der ganzen Versuchsdauer konstant gehalten. Die Abweichung gegenüber der natürlichen Temperatur im See überstieg nie $2{ }^{\circ} \mathrm{C}$. Während der Exposition betrugen die Temperaturschwankungen höchstens $\pm 0,5^{\circ} \mathrm{C}$.

Weitere $100 \mathrm{ml}$ wurden mit Lugolscher Lösung [27] fixiert, und der Rest diente zur chemischen Analyse. Es wurden dabei folgende Komponenten erfasst:

Aus dem Rohwasser: Gesamtphosphor, pH-Wert, SBV.

Aus dem Filtrat: Gesamtphosphor und Phosphat.

Im folgenden werden die Versuche vor allem beschreibend behandelt und die gewonnenen Resultate zusammengestellt. Eine eingehendere Diskussion und vergleichende Betrachtung der einzelnen Experimente folgt im nächsten Abschnitt.

\subsection{Versuch von 27.7.66 bis 14.8 .66}

Am Versuch waren vier Nährstofftestzellen beteiligt. Die Phosphorkonzentration wurde durch Zugabe von $\mathrm{NaH}_{2} \mathrm{PO}_{4} \cdot 2 \mathrm{H}_{2} \mathrm{O}$ in $\mathrm{NTZ}_{15}$ um $15 \mathrm{mg} / \mathrm{m}^{3}$, in $\mathrm{NTZ}_{30}$ um $30 \mathrm{mg} / \mathrm{m}^{3}$ und in $\mathrm{NTZ}_{50}$ um $50 \mathrm{mg} \mathrm{P} / \mathrm{m}^{3}$ erhöht. $\mathrm{NTZ}_{0}$ enthielt als Vergleichsprobe unverändertes Seewasser. Die Konzentrationsänderungen der drei Phosphorkomponenten $\mathrm{PO}_{4}$, GOP und PP sowie die Veränderungen im Bestand des Phytoplanktons wurden während 18 Tagen verfolgt und die Photosyntheseaktivität im Rotationslichtthermostaten unter konstanten Bedingungen gemessen.

\section{Zeitliche Veränderung der Konzentration der Phosphorkomponenten $\left(P_{4}, G O P, P P\right)$}

Im Laufe der Versuchszeit konnte in allen vier NTZ eine Abnahme der Gesamtphosphorkonzentration beobachtet werden (Abb. 11). Dieser Verlust an Gesamtphosphor schied sich in den Nährstofftestzellen $\left(\mathrm{NTZ}_{\mathbf{1 5}}, \mathrm{NTZ}_{\mathbf{3 0}}\right.$ und $\left.\mathrm{NTZ}_{50}\right)$ deutlich in zwei Phasen: eine rasche Abnahime während der ersten Tage und eine verlangsamte, konstante während der restlichen Versuchszeit. Diese Erscheinung kann verschiedene Ursachen haben:

- Verlust von gelösten Phosphorverbindungen durch ein Leck, durch Adsorption oder durch Diffusion.

- Aufnahme des $\mathrm{PO}_{4}$ durch Organismen und nachfolgender Verlust des PP durch Sedimentation.

Da nach Abschluss des Versuches keine Leckstelle festgestellt wurde und sich Adsorption und Diffusion, wie aus einem besonderen Versuch im Labor hervorging, kaum bemerkbar machen, muss der Grossteil des Gesamtphosphorverlusts auf Sedimentation von PP zurückgeführt werden. Die Gesamtphosphordefizite betrugen bei Versuchsabbruch in der $\mathrm{NTZ}_{0} 7 \mathrm{mg} \mathrm{P} / \mathrm{m}^{3}$, in der $\mathrm{NTZ}_{15} 23 \mathrm{mg} \mathrm{P} / \mathrm{m}^{3}$, in $\operatorname{der} \mathrm{NTZ} Z_{30}$ $37 \mathrm{mg} \mathrm{P} / \mathrm{m}^{3}$ und in der $\mathrm{NTZ}_{50} 57 \mathrm{mg} \mathrm{P} / \mathrm{m}^{3}$. Das zeigt deutlich, dass die Verlustraten konzentrationsabhängig sind, und es scheint, dass sich die Gesamtphosphorkonzentrationen der gedüngten Proben bei längerer Versuchsdauer wieder jener der $\mathrm{NTZ}_{0}$ angeglichen hätten.

In den $\mathrm{NTZ}_{15}, \mathrm{NTZ}_{30}$ und $\mathrm{NTZ}_{50}$ wurden nach dem ersten Versuchstag maximale PP-Konzentrationen festgestellt. Der absolute Betrag dieses Maximums steht mit der zugegebenen Phosphatmenge in einem annähernd linearen Zusammenhang. Nach dem Durchlaufen dieses Maximus fallen die PP-Kurven parallel zu den Gesamtphos- 

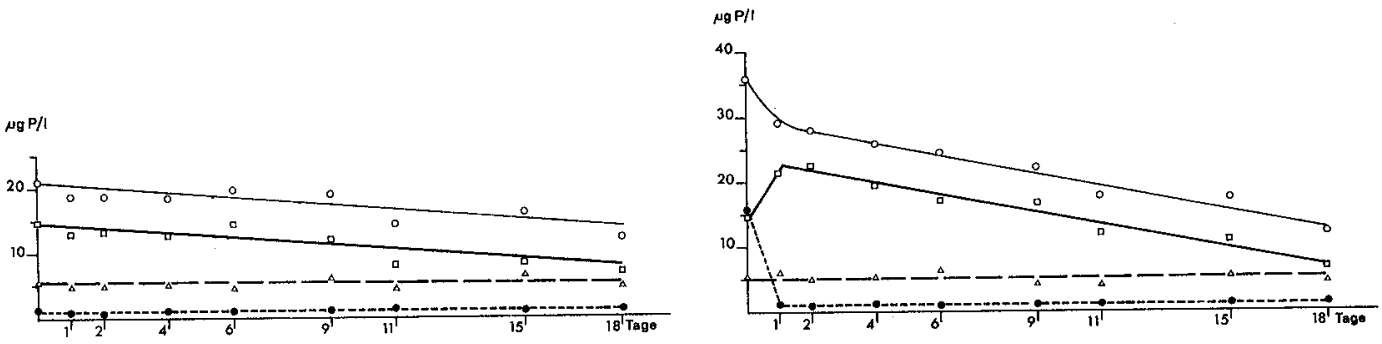
$N T Z_{0}$ $N Z_{15}$
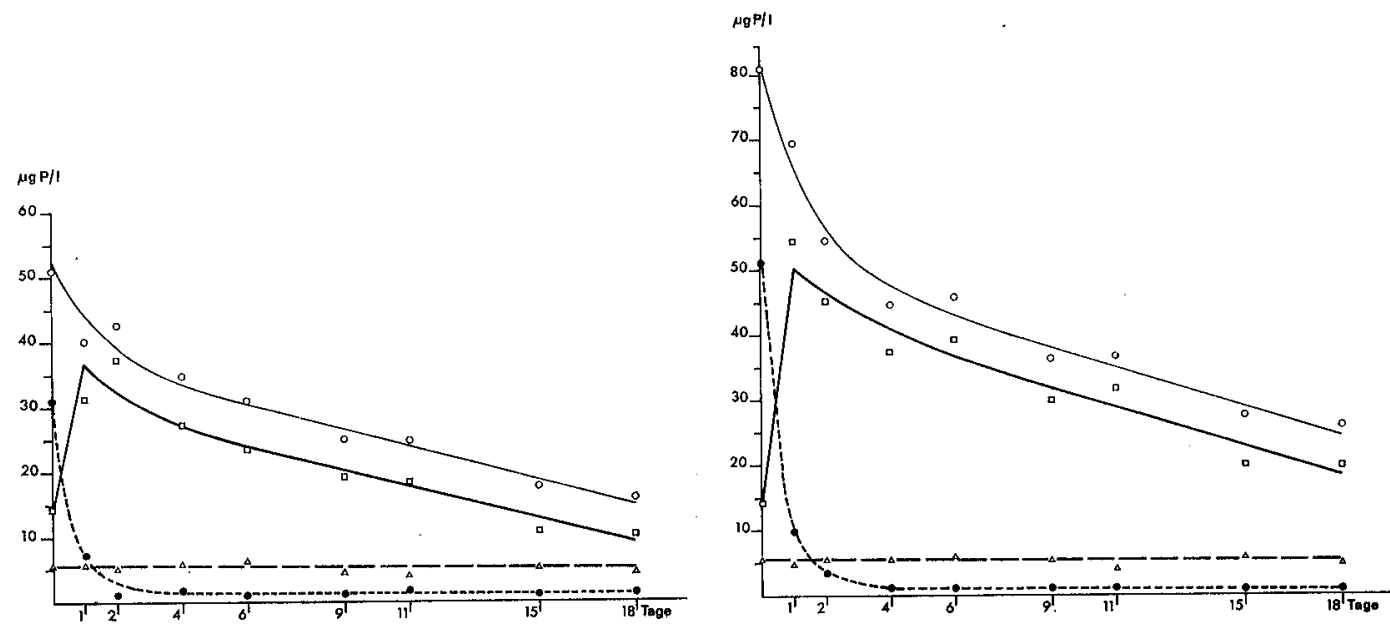
$\mathrm{NTZ}_{30}$ $\mathrm{NTZ}_{50}$

Abb. 11. Konzentrationsänderungen der Phosphorkomponenten $\mathrm{PO}_{4}-\mathrm{P}$ GOP $(\triangle-\ldots-\ldots), \operatorname{PP}(\square-\square, \square)$ sowie der Gesamtphosphorkonzentration $(\bigcirc-\ldots-O)$.

phorkurven ab, ein weiteres Indiz dafür, dass die Verluste an Gesamtphosphor auf Sedimentation von PP zurückzuführen sind. Während der Versuchsdauer wurde im See und in den NTZ eine sehr starke biogene Kalkausfällung beobachtet. Es muss in Betracht gezogen werden, dass ein Teil des zugesetzten Phosphats an der Oberfläche dieser Kalkpartikel adsorbiert und mit ihnen sedimentiert wurde. PP darf daher nicht unbesehen mit organismisch fixiertem Phosphor gleichgesetzt werden.

Das zugegebene Phosphat verschwand sehr rasch aus der Lösung und erreichte nach spätestens 4 Tagen in allen Nährstofftestzellen wieder den ursprünglichen Wert von etwa $1 \mathrm{mg} \mathrm{PO}_{4}-\mathrm{P} / \mathrm{m}^{3}$. Bei Versuchsbeginn ist die Abnahme der Phosphatkonzentration pro Zeiteinheit linear von der $\mathrm{PO}_{4}$-Konzentration zur Zeit $t_{0}$ abhängig.

Die Konzentration der GOP-Verbindungen schwankte in allen NTZ unabhängig von der Gesamtphosphorkonzentration um $5 \mathrm{mg} \mathrm{P} / \mathrm{m}^{3}$.

\section{Photosyntheseaktivität}

Die Kohlenstoffassimilation ist eine Funktion der momentanen qualitativen und quantitativen Zusammensetzung des Phytoplanktons und des Zusammenspiels aller 
äusseren Wirkungsfaktoren. Da sich bei längerer Versuchsdauer die Biomassen je nach den gegebenen Umweltbedingungen verschieden verändern, und es sich in unsern Experimenten zudem nicht um Monokulturen, sondern um heterogene, nicht synchrone, natürliche Populationen handelt, ist es unmöglich, die gemessenen Photosyntheseraten in eine bestimmte Anzahl "Assimilationseinheiten" einerseits und die momentane Wirkung der Umweltfaktoren andererseits zu zerlegen. Wenn es zutreffen sollte, dass unter Umständen eine erhöhte Assimilationsleistung mit verstärkter Exkretion gekoppelt ist oder Assimilationsprodukte als Reservestoffe angelegt werden, so wären hohe Assimilationsraten nur noch bedingt mit starkem Wachstum oder rascher Zellteilung identisch. Die gemessenen Aktivitäten sind zwar ein Mass für die Produktion an organischer Substanz und damit eine integrierte Antwort des Systems auf die veränderten Umweltbedingungen, aber sie sagen weder über den momentanen Stand der Nährstoffverhältnisse noch über qualitative oder quantitative Veränderungen der Zusammensetzung des Phytoplanktons etwas aus.

Die im ersten Versuch gemessenen Photosyntheseraten sind in Tab. 16 zusammengestellt.

Tab. 16. Photosyntheseraten (Angaben in $\mathrm{mg} \mathrm{C}_{\text {ass }} / \mathrm{m}^{3} \cdot \mathrm{h}$ ).

\begin{tabular}{|c|c|c|c|c|c|c|c|c|}
\hline \multirow[t]{2}{*}{ NTZ } & \multicolumn{8}{|c|}{ Versuchstag } \\
\hline & 1. & 2. & 4. & 6. & 9. & 11. & 15. & 18. \\
\hline 0 & 2,16 & 3,19 & 4,43 & 4,84 & 3,20 & 2,06 & 2,14 & 2,26 \\
\hline 15 & 4,05 & 6,96 & 7,82 & 9,40 & 3,91 & 3,75 & 2,43 & 2,46 \\
\hline 30 & 5,06 & 9,75 & 13,40 & 11,55 & 5,95 & 6,16 & 5,10 & 3,71 \\
\hline 50 & 6,76 & 10,60 & 15,80 & 25,23 & 13,89 & 13,09 & 5,98 & 5,85 \\
\hline
\end{tabular}

Aus diesen Ergebnissen geht hervor, dass steigende Phosphatdüngung eine steigende Photosyntheseleistung bewirkte. In allen Nährstofftestzellen wurden zwischen dem vierten und sechsten Versuchstag maximale Assimilationsraten gemessen. Da zu diesem Zeitpunkt die künstlich erhöhten Phosphatspiegel bereits wieder auf die ursprüngliche, natürliche Konzentration (etwa $1 \mathrm{mg} \mathrm{PO}_{4}-\mathrm{P} / \mathrm{m}^{3}$ ) abgesunken waren, muss angenommen werden, dass das zugesetzte Phosphat vom Plankton momentan aufgenommen und gespeichert, aber erst später wirksam wurde. Nach dem sechsten Versuchstag fielen die Photosyntheseraten in den gedüngten Proben wieder rasch ab, und ihr Verlauf lässt erwarten, dass sie sich bei längerer Versuchsdauer wieder jenen der Vergleichsprobe angeglichen hätten.

\section{Qualitative und quantitative Planktonanalysen}

Weil wie erwähnt die Messung der Photosyntheseraten allein keine eindeutigen Schlüsse auf Wachstum und Vermehrung der planktischen Algen zulässt und es zudem interessant ist, die Reaktion der wichtigsten Phytoplankter auf verschieden starke Phosphordüngung kennenzulernen, wurden jene Vertreter, die das planktische Bild dominierten, im umgekehrten Mikroskop ausgezählt. Dabei wurden nur jene Formen berücksichtigt, die so häufig auftraten, dass der reine Zählfehler die $20 \%$-Grenze nicht überstieg [61]. Bei der Beurteilung der Resultate muss zudem berücksichtigt werden, dass jede entnommene Probe wegen der möglichen inhomogenen Verteilung des Planktons in den NTZ mit einem nicht generell bestimmbaren Fehler behaftet ist. 
Die Resultate der Analyse des Phytoplanktons sind in Tab. 17 zusammengestellt. Es geht daraus deutlich hervor, dass die verschiedenen Vertreter des Phytoplanktons auf die Phosphatdüngungen verschieden reagierten.

Für Oscillatoria rubescens waren in der Vergleichszelle nach dem sechsten Versuchstag die Verlustraten grösser als die Zuwachsraten. In den $\mathrm{NTZ}_{15}, \mathrm{NTZ}_{\mathbf{3 0}}$ und $\mathrm{NTZ}_{50}$ entwickelte sie sich deutlich besser als in der Kontrolle, aber die Wachstumsraten unterschieden sich bei verschieden starker Düngung nicht mehr signifikant voneinander. Stephanodiscus spp. und Cyclotella spp. erreichten am sechsten Versuchstag in allen NTZ maximale Individuenzahlen. In den gedüngten Proben waren

Tab. 17. Individuendichten (Angaben in Individuen pro Liter).

Oscillatoria mbescens

\begin{tabular}{|c|c|c|c|c|c|}
\hline NTZ & $\begin{array}{l}\text { Versuchstag } \\
\text { 1. Tag }\end{array}$ & 6. Tag & 11. Tag & 15. Tag & 18. Tag \\
\hline 0 & 20000 & 21600 & 8600 & 7850 & 7860 \\
\hline 15 & 16650 & 25320 & 59000 & 59000 & 17240 \\
\hline 30 & 17650 & 18000 & 41.000 & 70600 & 34000 \\
\hline 50 & 20000 & 42000 & 58800 & 54800 & 27520 \\
\hline
\end{tabular}

Stephanodiscus spp. und Cyclotella spp.

\begin{tabular}{|c|c|c|c|c|}
\hline NTZ & $\begin{array}{l}\text { Versuchstag } \\
\text { 1. Tag }\end{array}$ & 6. Tag & 11. Tag & 15. Tag \\
\hline 0 & 1335000 & 1552000 & 740000 & 196000 \\
\hline 15 & 1400000 & 2720000 & 886000 & 201000 \\
\hline 30 & 1530000 & 2384000 & 334000 & 133000 \\
\hline 50 & 1295000 & 3108000 & 288000 & 137000 \\
\hline
\end{tabular}

\begin{tabular}{|c|c|c|c|c|c|}
\hline $\mathrm{NTZ}$ & $\begin{array}{l}\text { Versuc } \\
\text { 1. Tag }\end{array}$ & 6. Tag & 11. Tag & 15. Tag & 18. Tag \\
\hline \multicolumn{6}{|c|}{ Nephrocytium sp. } \\
\hline 0 & 4800 & 6000 & 7350 & 6870 & 7000 \\
\hline 15 & 3800 & 7850 & 19600 & $\dagger$ & 20000 \\
\hline 30 & 4920 & 4000 & 29500 & 49200 & 34800 \\
\hline 50 & 4000 & 13500 & 24380 & 44000 & 47160 \\
\hline \multicolumn{6}{|c|}{ Mougeotia sp. } \\
\hline 0 & 24550 & 24000 & 58700 & 60900 & 71800 \\
\hline 15 & 24600 & 50900 & 83800 & 167000 & 160000 \\
\hline 30 & 20000 & 40000 & 133400 & 133000 & 290000 \\
\hline 50 & 30000 & 78000 & 80400 & 147800 & 166000 \\
\hline \multicolumn{6}{|c|}{ Ulothrix amphigranulata } \\
\hline 0 & 24550 & 31000 & 103000 & 84400 & 100000 \\
\hline 15 & 27200 & 162000 & 182000 & 216000 & 141000 \\
\hline 30 & 21.300 & 148000 & 261000 & 410000 & 527000 \\
\hline 50 & 19500 & 294000 & 570000 & 417000 & 491000 \\
\hline
\end{tabular}


sie ungefähr 1,5-2mal so hoch wie bei Versuchsbeginn. In der Kontrolle dagegen konnte kaum eine gesicherte Zunahme festgestellt werden. Bei den Grünalgen Nephrocytium sp., Ulothrix amphigranulata und Mougeotia sp. konnte in den gedüngten Proben eine eindeutige Steigerung der Vermehrungsraten beobachtet werden. Während die Individuenzahl von Nephrocytium in der Vergleichszelle kaum zunahm, wurde am Versuchsende in der $\mathrm{NTZ}_{50}$ rund der 12fache Ausgangswert registriert. Bei Mougeotia steht dem Quotienten $I_{18} / I_{0}$ von $Q=3$ in der Vergleichszelle ein solcher von $Q=15$ in der $\mathrm{NTZ}_{30}$ gegenüber. In der $\mathrm{NTZ}_{\mathbf{5 0}}$ entwickelte sich diese Alge bereits wieder schlechter als in $\mathrm{NTZ}_{30}$, aber immer noch deutlich besser als in der Vergleichsprobe. Ulothrix vermehrte sich um so stärker, je höher die Anfangsphosphatkonzentration war. Einem Nettozuwachs von rund 75000 Fäden pro Liter in der Kontrolle steht ein solcher von rund 480000 Fäden pro Liter in der $\mathrm{NTZ}_{\mathbf{3 0}}$ und $\mathrm{NTZ}_{\mathbf{5 0}}$ gegenüber.

\subsection{Versuch vom 11.10 .66 bis 20.10 .66}

An dieser Versuchsserie waren drei Nährstofftestzellen beteiligt. Die natürliche Phosphorkonzentration der Vergleichsprobe $\left(\mathrm{NTZ}_{0}\right)$ wurde in der $\mathrm{NTZ} Z_{10}$ um $10 \mathrm{mg}$ $\mathrm{PO}_{4}-\mathrm{P} / \mathrm{m}^{3}$ und in der $\mathrm{NTZ}_{30}$ um $30 \mathrm{mg} \mathrm{PO}_{4}-\mathrm{P} / \mathrm{m}^{3}$ erhöht. Da durch sehr starken Wellengang die Rührstangen der drei NTZ beschädigt wurden, musste das Experiment bereits am neunten Versuchstag abgebrochen werden.

Zeitliche Veränderungen der drei Phosphorkomponenten $\left(\mathrm{PO}_{4}, \mathrm{GOP}, \mathrm{PP}\right)$

Im Gegensatz zum ersten Experiment blieb diesmal die Gesamtphosphorkonzentration in allen drei NTZ während der ganzen Versuchsdauer konstant (Abb. 12).
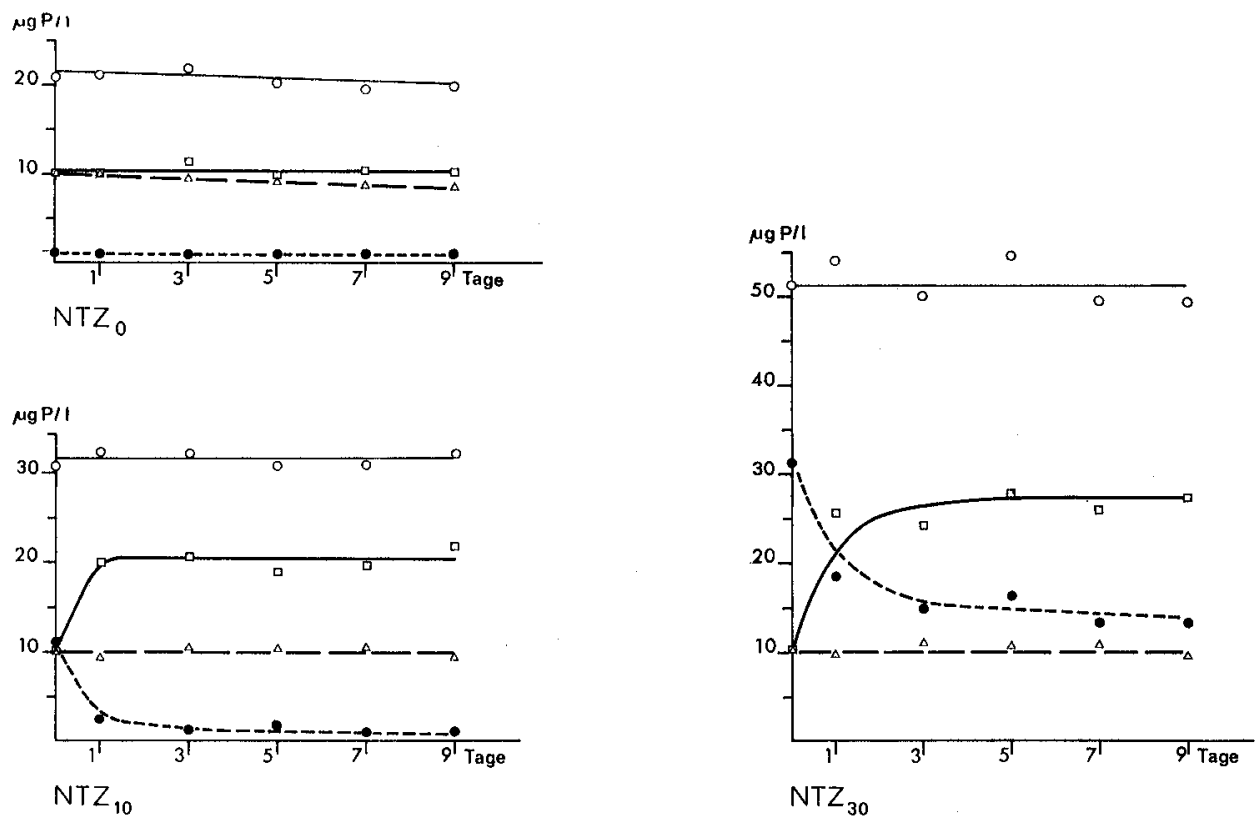

Abb. 12. Konzentrationsänderungen der Phosphorkomponenten $\mathrm{PO}_{4} \mathrm{P}($................ GOP $(\triangle----\triangle)$, PP $(\square-\square)$ sowie der Gesamtphosphorkonzentration $(0-\square)$ 
Das zugesetzte Phosphat verschwand während der ersten Versuchstage rasch aus der Lösung, konnte aber quantitativ in partikulärer Form wieder gefunden werden. Im weiteren unterschied sich dieses Experiment vom vorhergehenden eindeutig dadurch, dass das zugegebene Phosphat nicht vollständig in PP übergeführt wurde. In der $\mathrm{NTZ}_{30}$ verblieb in der neuen Gleichgewichtslage eine Restkonzentration von rund $14 \mathrm{mg} \mathrm{PO}_{4}-\mathrm{P} / \mathrm{m}^{3}$, und die $\mathrm{PO}_{4}$-Aufnahmerate am ersten Tag fiel diesmal besonders in der $\mathrm{NTZ}_{30}$ wesentlich niedriger aus als im Sommer. Die GOP-Konzentration lag mit $10 \mathrm{mg} \mathrm{P} / \mathrm{m}^{3}$ in allen drei Zellen wesentlich höher als im ersten Versuch.

\section{Photosyntheseaktivität}

Auch in diesen Versuchsreihen wurden in den gedüngten Proben höhere Assimilationsraten gemessen als in der Verlgeichsprobe (Tab. 18). Sie übertrafen die Kontrolle am ersten Versuchstag um rund 300\%. Auffallend ist jedoch, dass nach weiterer Phosphataufnahme die Photosyntheseraten der $\mathrm{NTZ}_{\mathbf{3 0}}$ zwar grösser als jene der Kontrolle, aber kleiner als jene der $N T Z_{10}$ ausfielen. Daraus muss geschlossen werden, dass $\mathrm{zu}$ diesem Zeitpunkt mit einer $30-\mathrm{mg}-\mathrm{PO}_{4}-\mathrm{P} / \mathrm{m}^{3}$-Düngung der optimale Gehalt an Gesamtphosphor bereits überschritten wurde.

Tab. 18. Photosyntheseraten (Angabe in $\mathrm{mg} \mathrm{C}_{\text {ass }} / \mathrm{m}^{3} \cdot \mathrm{h}$ ).

\begin{tabular}{rlllll}
\hline NTZ & Versuchstag & & & \\
& 1. & 3. & 5. & 7. & 9. \\
\hline 0 & 1,13 & 2,46 & 2,30 & 2,00 & 3,16 \\
10 & 4,42 & 5,35 & 5,91 & 7,69 & 7,20 \\
30 & 4,41 & 4,11 & 4,34 & 4,32 & 4,24 \\
\hline
\end{tabular}

\section{Qualitative und quantitative Planktonanalyse}

Die häufigsten Formen waren zur Versuchszeit Oscillatoria rubescens, Gomphosphaeria lacustris und Tabellaria fenestrata. Die Fadenlänge von Oscillatoria rubescens war in diesem Versuch in $\mathrm{NTZ}_{\mathbf{0}}$ während der ganzen Versuchsdauer wesentlich grösser als in den beiden gedüngten Proben. Eine Erklärung dafür kann nicht gegeben werden; es ist aber daran zu denken, dass das Wasser der verschiedenen NTZ möglicherweise nicht aus demselben Wasserkörper und aus genau derselben Tiefe stammt. In Tab. 19 wurde aus diesem Grunde neben der Anzahl Individuen pro Liter für diese Alge auch noch die Biomasse $\left(\mathrm{mm}^{3} / \mathrm{l}\right)$ angegeben. Bis zum Abschluss des Versuchs hatten sich die Biomassen von Oscillatoria rubescens in den beiden gedüngten Proben verdreifacht. In der Vergleichszelle $\mathrm{NTZ}_{\mathbf{0}}$ konnte dagegen keine Vermehrung festgestellt werden. Oscillatoria reagierte also wie im ersten Experiment mit verstärktem Wachstum auf die Phosphatdüngung. Gomphosphaeria lacustris reagierte auf die Düngung mit $10 \mathrm{mg} \mathrm{PO}_{4}-\mathrm{P} / \mathrm{m}^{3}$ nicht signifikant, wurde jedoch durch die 30-mg- $\mathrm{PO}_{4}-\mathrm{P} / \mathrm{m}^{3}$-Düngung in der Vermehrung gehemmt. Die unterschiedliche Individuenzahl zwischen der $\mathrm{NTZ}_{0}$ und der $\mathrm{NTZ}_{10}$ am neunten Versuchstag ist wahrscheinlich auf eine erhöhte Verlustrate in der $\mathrm{NTZ}_{\mathbf{0}}$, verursacht durch den Ausfall des Rührers zwischen dem siebten und achten Tag, zurückzuführen. Dasselbe dürfte auch für Tabellaria fenestrata zutreffen. Wenn der Wert für den neunten Tag und $\mathrm{NTZ}_{0}$ korrigiert wird, so streuen die Werte nur innerhalb des methodisch mögli- 
chen Fehlers. Tabellaria reagierte in diesem Versuch also weder positiv noch negativ auf die Düngung mit Natriumdihydrogenphosphat.

Tab. 19.

Oscillatoria rubescens (Individuen pro Liter)

\begin{tabular}{|c|c|c|c|}
\hline \multirow[t]{2}{*}{ NTZ } & \multicolumn{2}{|c|}{ Versuchstag } & \multirow[b]{2}{*}{ 9. Tag } \\
\hline & 1. Tag & 5. Tag & \\
\hline 0 & 12000 & 37100 & 26000 \\
\hline 10 & 8800 & 23200 & 37700 \\
\hline 30 & 10200 & 22000 & 18900 \\
\hline \multicolumn{4}{|c|}{ Tabellaria fenestrata (Individuen pro Liter) } \\
\hline 0 & 43600 & 75900 & 30000 \\
\hline 10 & 34000 & 61800 & 64500 \\
\hline 30 & 34500 & 48600 & 89000 \\
\hline
\end{tabular}

\begin{tabular}{|c|c|c|c|}
\hline \multirow[t]{2}{*}{$\mathrm{NTZ}$} & \multicolumn{3}{|c|}{ Versuchstag } \\
\hline & 1. Tag & 5. Tag & 9. Tag \\
\hline 0 & 33000 & 31800 & 27300 \\
\hline 10 & 28300 & 23200 & 41000 \\
\hline 30 & 22100 & 32000 & 84000 \\
\hline \multicolumn{4}{|c|}{ Oscillatoria rubescens $\left(\mathrm{mm}^{3} / 1\right)$} \\
\hline 0 & 0.7 & 0,8 & 0,6 \\
\hline 10 & 0,3 & 0,5 & 1,0 \\
\hline 30 & 0,3 & 0,5 & 0,9 \\
\hline
\end{tabular}

\subsection{Versuch vom 16.12.66 bis 5.1.67}

An diesem Versuch waren vier Nährstofftestzellen beteiligt: $\mathrm{NTZ}_{\mathbf{0}}, \mathrm{NTZ}_{5}, \mathrm{NTZ}_{\mathbf{1 0}}$ und $\mathrm{NTZ}_{\mathbf{3 0}}$. (Die $\mathrm{Zahl}$ im Index gibt die zugesetzte Phosphatmenge in $\mathrm{mg} / \mathrm{m}^{\mathbf{3}}$ an). Da die Plastikfolie im kalten Wasser relativ brüchig wird und während der Versuchsdauer stürmisches Wetter herrschte, traten bis zum Versuchsabbruch in allen Zellen ein bis zwei Risse von 1-2 cm Länge auf.

Zeitliche Veränderung der Konzentration der Phosphorkomponenten $\left(P_{4}, G O P, P P\right)$

Bei den Tauchgängen konnten die erwähnten kleinen Leckstellen nicht beobachtet werden. Es ist daher ungewiss, wann sie auftraten, und es muss damit gerechnet werden, dass durch sie ein unbekannter Anteil des zugesetzten Phosphats in den See diffundierte. Aus den absoluten Konzentrationsänderungen der gelösten Phosphorverbindungen dürfen daher keine Schlüsse gezogen werden.

Immerhin fällt auf (vgl. Abb. 13), dass die PP-Konzentrationen der verschiedenen Nährstofftestzellen trotz stark unterschiedlichem Phosphatgehalt nur sehr wenig voneinander abwichen. Die Umsetzung von zugegebenem Phosphat in PP erfolgte somit während der Versuchszeit nur sehr langsam. Das bedeutet, dass der Phosporbedarf und das Speicherungsvermögen des Phytoplanktons sehr gering waren. Die GOPKonzentration schwankte in allen Zellen unabhängig von der Phosphatkonzentration zwischen 8 und $10 \mathrm{mg} \mathrm{P} / \mathrm{m}^{3}$.

\section{Photosyntheseaktivität}

Die Assimilationsleistungen des Phytoplanktons variierten in der $\mathrm{NTZ}_{0}, \mathrm{NTZ}_{10}$ und $\mathrm{NTZ}_{30}$ zwischen 1,8 und $2,8 \mathrm{mg} \mathrm{C} \mathrm{C}_{\text {ass }} / \mathrm{h} \cdot \mathrm{m}^{3}$ und unterschieden sich nicht signifikant voneinander. In der $\mathrm{NTZ}_{5}$ wurden im mittleren Versuchsabschnitt, wie aus Tab. 20 hervorgeht, an fünf aufeinanderfolgenden Versuchstagen leicht erhöhte Photosyntheseraten gemessen. Die höchste Assimilationsrate $\left(3,49 \mathrm{mg} \mathrm{C} / \mathrm{h} \cdot \mathrm{m}^{3}\right)$ wurde in der $\mathrm{NTZ}_{\mathbf{5}}$ am elften Versuchstag beobachtet. 

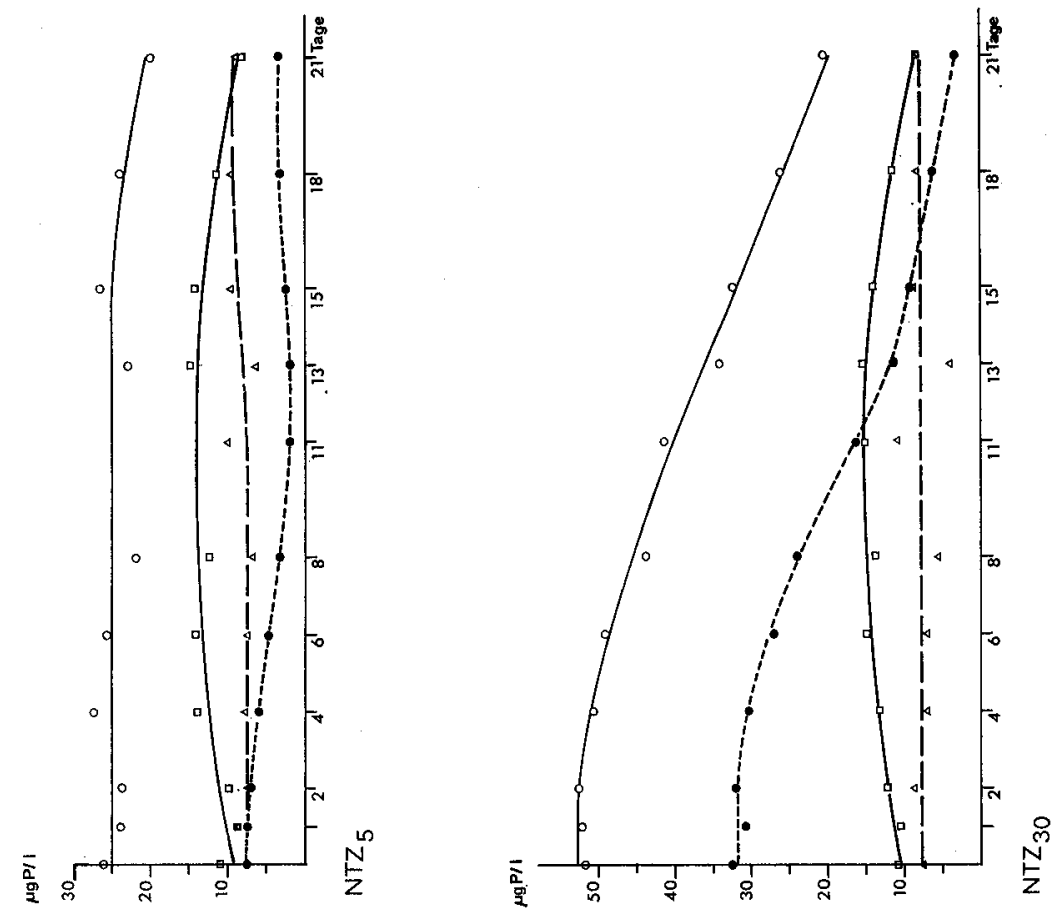

के
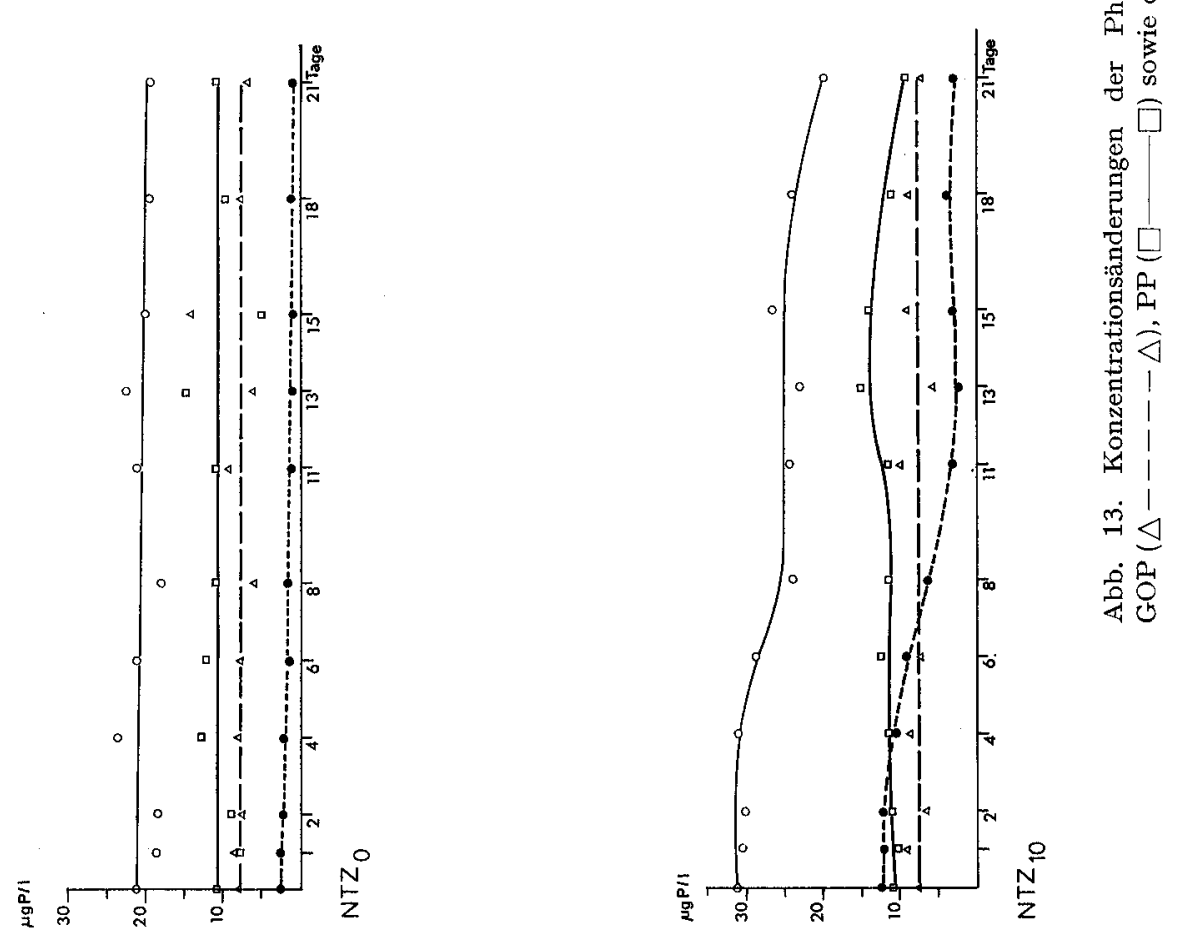
Während des Versuches in den Monaten Dezember/Januar konnte mit einer Phosphatdüngung also keine gesicherte Erhöhung der Primärproduktion bewirkt werden. Es muss daher angenommen werden, dass im Winter andere Faktoren, vor allem die niedrige Temperatur und das relativ schwache Licht, die Produktion an organischer Substanz begrenzen.

Tab. 20. Photosyntheseaktivität (Angaben in $\mathrm{mg} \mathrm{C}_{\mathrm{ass}} / \mathrm{h} \cdot \mathrm{m}^{3}$ ).

\begin{tabular}{rllllllllll}
\hline NTZ & \multicolumn{2}{c}{ Versuchstag } & & & & & & & & \\
& 1. & 2. & 4. & 6. & 8. & 11. & 13. & 15. & 18. & 21. \\
\hline 0 & 2,29 & 2,23 & 2,22 & 2,68 & 2,58 & 2,72 & 2,80 & 1,93 & 1,83 & 2,66 \\
5 & 2,14 & 1,96 & 2,22 & 2,80 & 3,08 & 3,49 & 3,16 & 3,09 & 2,58 & 2,35 \\
10 & 2,32 & 2,15 & 2,29 & 2,41 & 2,52 & 2,68 & 2,50 & 2,69 & 2,50 & 2,30 \\
30 & 2,33 & 2,02 & 2,46 & 2,50 & 2,54 & 2,51 & 1,83 & 2,20 & 2,04 & 2,40 \\
\hline
\end{tabular}

Tab. 21. Individuen pro Liter.

\begin{tabular}{|c|c|c|c|c|}
\hline NTZ & $\begin{array}{l}\text { Versuchstag } \\
\text { 2. Tag }\end{array}$ & 6. Tag & 11. Tag & 21. Tag \\
\hline \multicolumn{5}{|c|}{ Oscillatoria rubescens } \\
\hline 0 & 24000 & 24000 & 30000 & 24000 \\
\hline 5 & 26000 & 25000 & 28000 & 33000 \\
\hline 10 & 31000 & 24000 & 27000 & 25000 \\
\hline 30 & 25000 & 31000 & 30000 & 31000 \\
\hline \multicolumn{5}{|c|}{ Stephanodiscus spp. (St. hantzschii und St. alpinus) } \\
\hline 0 & 51000 & 83000 & 120000 & 75000 \\
\hline 5 & 53000 & 82000 & 92000 & 74000 \\
\hline 10 & 58000 & 85000 & 81000 & 58000 \\
\hline 30 & 56000 & 84000 & 99000 & 80000 \\
\hline \multicolumn{5}{|c|}{ Tabellaria fenestrata } \\
\hline 0 & 45000 & 95000 & 160000 & 120000 \\
\hline 5 & 52000 & 100000 & 140000 & 100000 \\
\hline 10 & 43000 & 77000 & 100000 & 72000 \\
\hline 30 & 56000 & 100000 & 110000 & 91000 \\
\hline \multicolumn{5}{|c|}{ Asterionella formosa } \\
\hline 0 & 46000 & 63000 & 140000 & 100000 \\
\hline 5 & 36000 & 51000 & 50000 & 79000 \\
\hline 10 & 46000 & 54000 & 79000 & 58000 \\
\hline 30 & 33000 & 80000 & 86000 & 66000 \\
\hline \multicolumn{5}{|c|}{ Synedra cf. berolinensis } \\
\hline 0 & 28000 & 33000 & 67000 & 150000 \\
\hline 5 & 33000 & 37000 & 72000 & 170000 \\
\hline 10 & 38000 & 36000 & 56000 & 120000 \\
\hline 30 & 31000 & 35000 & 30000 & 180000 \\
\hline
\end{tabular}


Qualitative und quantitative Planktonanalyse

Oscillatoria rubescens, Tabellaria fenestrata, Asterionella formosa, Synedra cf. berolinensis und Stephanodiscus spp. waren während der Versuchszeit die wichtigsten Vertreter des Phytoplanktons. Wie aus Tab. 21 hervorgeht, entwickelte sich in den gedüngten Proben keine Form besser als in der Vergleichszelle. Die Wachstums- und Vermehrungsraten waren (in guter Übereinstimmung mit der gemessenen Photosyntheseaktivität) ungefähr gleich gross wie oder nicht signifikant kleiner als in der Kontrollzelle.

\subsection{Versuch vom 2.3.67 bis 17.3.67}

Am Versuch waren die vier Nährstofftestzellen $\mathrm{NTZ}_{\mathbf{0}}, \mathrm{NTZ}_{\mathbf{5}}, \mathrm{NTZ}_{\mathbf{1 0}}$ und $\mathrm{NTZ}_{\mathbf{3 0}}$ beteiligt. Die Zahl im Index gibt an, um wie viele $\mathrm{mg} \mathrm{PO}_{\mathbf{4}}-\mathrm{P} / \mathrm{m}^{3}$ die Anfangskonzentration durch Zugabe von Natriumdihydrogenphosphat erhöht wurde.

\section{Zeitliche Veränderung der Konzentrationen der Phosphorkomponenten $\left(\mathrm{PO}_{4}, \mathrm{GOP}, \mathrm{PP}\right)$}

Im Gegensatz zum Experiment von Abschnitt 4.3 im Dezember/Januar konnte in diesem Versuch wieder eine deutliche Überführung von Phosphat in PP beobachtet werden. Die zeitliche Veränderung der Phosphatkonzentration (Abb. 14) unterschied sich aber von jener der Sommer- und Herbstexperimente (Abschnitte 4.1, 4.2, 4.5, 4.6) dadurch, dass die Aufnahmegeschwindigkeit zur Zeit Null minimal war und je nach der vorgelegten Anfangskonzentration erst in einem späteren Zeitpunkt ihr Maximum erreichte.

Das rasche Anwachsen der PP-Konzentration im mittleren Versuchsabschnitt fiel zeitlich mit der grössten Zunahme der Assimilationsraten und der stärksten Vermehrung der Zellzahlen zusammen. Es wäre verlockend, aus diesen Beobachtungen auf ein mit Phosphat optimal versorgtes Phytoplankton mit kleinem Speicherungsvermögen zu schliessen (s. S. 54).

Die künstliche $\mathrm{PO}_{4}$-Erhöhung wirkte sich auch in diesem Experiment nicht auf die Konzentration des GOP aus. Sie schwankte, ohne sichtlichen Zusammenhang mit der $\mathrm{PO}_{4}$-Konzentration, um 7,5 $\mu \mathrm{g} / 1$.

\section{Photosyntheseaktivität}

Bis zum sechsten Versuchstag unterschieden sich die Photosyntheseraten der vier NTZ nicht signifikant voneinander. Verglichen mit der $\mathrm{NTZ}_{0}$, wurden am elften und dreizehnten Tage in der $\mathrm{NTZ}_{\mathbf{1 0}}$ die doppelten, am fünfzehnten Tag gar die vierfachen Assimilationsraten erreicht. Die Assimilationsraten der $\mathrm{NTZ}_{\mathbf{3 0}}$ lagen zwischen jenen der $\mathrm{NTZ}_{10}$ und der Vergleichszelle $\mathrm{NTZ}_{\mathbf{0}}$. Die für das Phytoplankton optimale PKonzentration war offenbar auch in diesem Versuch durch die Düngung mit $30 \mathrm{mg}$ $\mathrm{P} / \mathrm{m}^{3}$ bereits überschritten. Die Photosyntheseraten der $\mathrm{NT} Z_{5}$ lagen anfänglich leicht tiefer, später unbedeutend höher als jene der Vergleichszelle.

\section{Quantitative und qualitative Planktonanalyse}

Oscillatoria rubescens vermehrte sich in allen vier NTZ kaum. Ein Einfluss der Phosphatdüngung konnte nicht sicher beobachtet werden. Tabellaria fenestrata erreichte in den gedüngten Proben am zwölften Tag ihre maximale Dichte. $\mathrm{Zu}$ diesem 

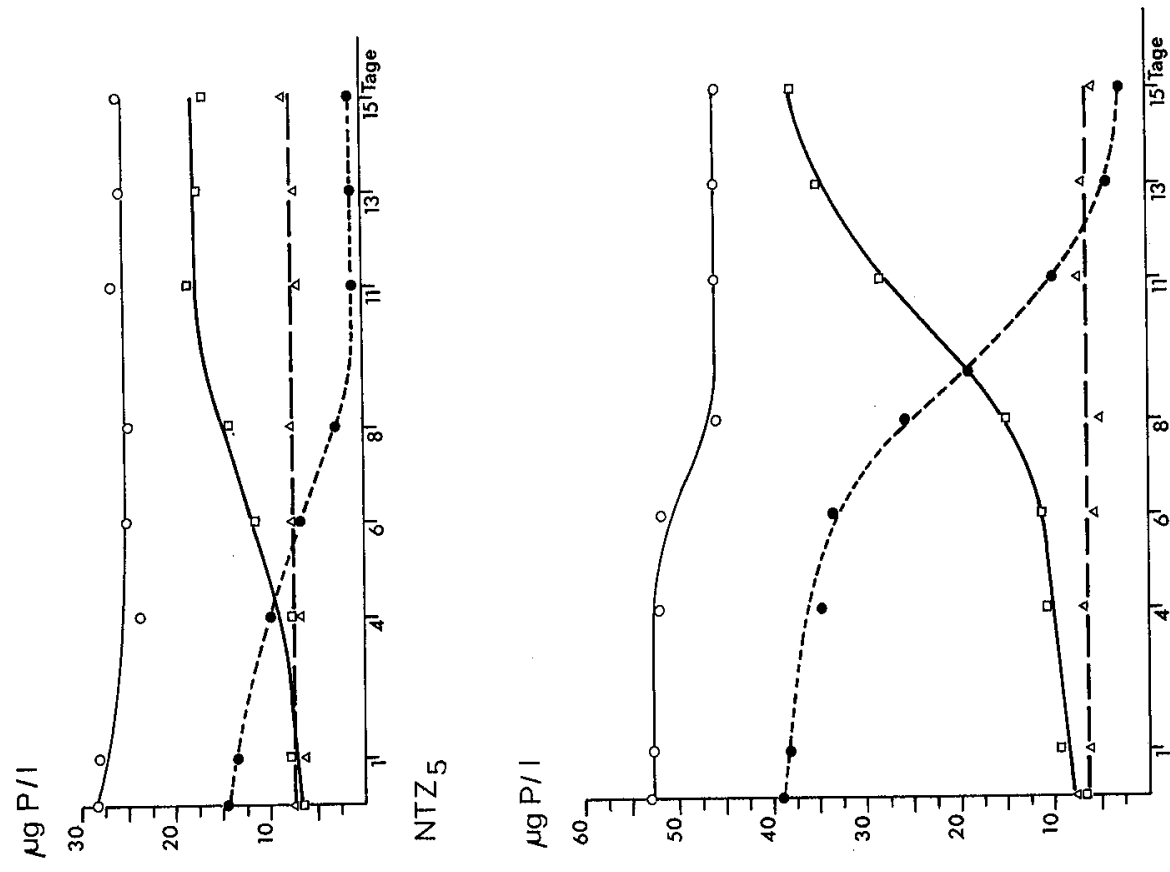

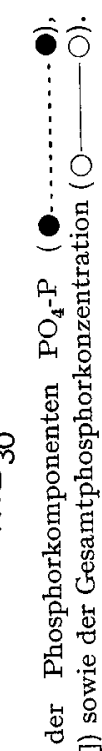
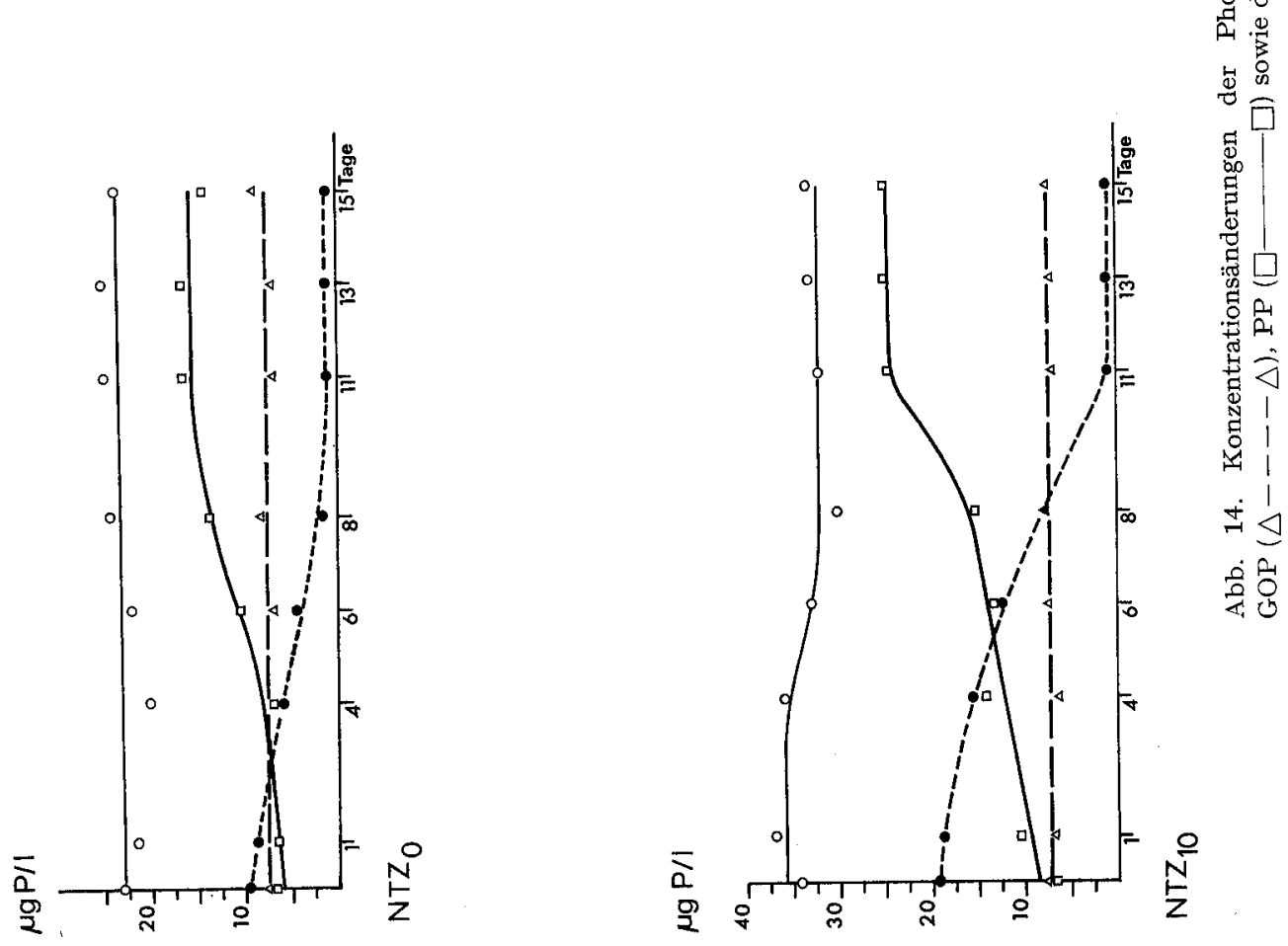
Zeitpunkt wurde in der $\mathrm{NTZ}_{10}$ beinahe, in der $\mathrm{NTZ}_{\mathbf{3 0}}$ mehr als die doppelte Zellzahl registriert. Positiv auf die Düngung reagierte auch Synedra cf. berolinensis, die, verglichen mit der Kontrolle am zwölften Tag, in der $\mathrm{NTZ}_{\mathbf{1 0}}$ beinahe die dreifache und in der $\mathrm{NTZ}_{30}$ gut die doppelte Dichte erreichte. Stephanodiscus astraea übertraf am zwölften Tag in den Nährstofftestzellen $\mathrm{NTZ}_{\mathbf{1 0}}$ und $\mathrm{NTZ}_{\mathbf{3 0}}$ die Individuenzahl der Vergleichszelle um mehr als $100 \%$, und Stephanodiscus hantzschii entwickelte sich in der $\mathrm{NTZ}_{10}$ am besten, wo er bei Versuchsabbruch mit 17500000 Individuen pro Liter mehr als den doppelten Wert der Kontrolle erreichte. Mit Ausnahme von Oscillatoria rubescens reagierten also alle berücksichtigten Formen eindeutig positiv auf die Düngung.

Tab. 22. Photosyntheseaktivität (Angaben in $\mathrm{mg} \mathrm{C}_{\text {ass }} / \mathrm{h} \cdot \mathrm{m}^{3}$ ).

\begin{tabular}{|c|c|c|c|c|c|c|c|}
\hline \multirow[t]{2}{*}{ NTZ } & \multicolumn{7}{|c|}{ Versuchstag } \\
\hline & 1 . & 4. & 6. & 8. & 11. & 13. & 15. \\
\hline 0 & 1,99 & 2,14 & 4,40 & 5,25 & 6,74 & 6,62 & 4,40 \\
\hline 5 & 2,02 & 2,26 & 3,27 & 4,00 & 7,32 & 6,76 & 7,70 \\
\hline 10 & 1,91 & 2,38 & 3,66 & 5,98 & 14,94 & 14,60 & 17,08 \\
\hline 30 & 1,86 & 2,36 & 3,80 & 4,75 & 9,07 & 7,70 & 11,25 \\
\hline
\end{tabular}

\subsection{Versuch vom 28.4.67 bis 16.5 .67}

Diesmal wurde die Versuchsanordnung etwas anders gewählt als in den vorangehenden Experimenten. Um den Einfluss einer einmaligen massiven Düngung mit demjenigen einer schwächeren, aber mehrmals wiederholten Nährstoffanreicherung vergleichen zu können, wurde neben der normal ansteigenden Konzentrationsreihe $\mathrm{NTZ}_{\mathbf{0}}, \mathrm{NTZ}_{\mathbf{1 0}}, \mathrm{NTZ}_{\mathbf{3 0}}$ der vierten Nährstofftestzelle $\mathrm{NTZ}_{\mathbf{3} \times \mathbf{7}}$ während der Versuchsdauer von 19 Tagen $7 \mathrm{mal} 3 \mathrm{mg} \mathrm{PO}_{4}-\mathrm{P} / \mathrm{m}^{3}$ zugesetzt.

\section{Zeitliche Veränderung der Phosphorkomponenten $\left(\mathrm{PO}_{4}, \mathrm{GOP}, \mathrm{PP}\right)$}

Im ersten Zeitabschnitt blieb die Gesamt-P-Konzentration konstant und fiel nachher ungefähr parallel zur Konzentration des PP ab. Die Gesamt-P-Defizite betrugen beim Versuchsabbruch in der $\mathrm{NTZ}_{0} 5,5 \mathrm{mg} \mathrm{P} / \mathrm{m}^{3}$, in der $\mathrm{NTZ}_{10} 12,3 \mathrm{mg} \mathrm{P} / \mathrm{m}^{3}$ und in der $\mathrm{NTZ}_{\mathbf{3} 0} 20,0 \mathrm{mg} \mathrm{P} / \mathrm{m}^{3}$. Analog dazu stieg die Gesamt-P-Konzentration in der $\mathrm{NTZ}_{\mathbf{3} \times \mathbf{7}}$ während der ersten 7 Tage an und blieb nachher ungefähr konstant.

Das zugesetzte $\mathrm{PO}_{4}$ wurde in der $\mathrm{NTZ}_{30}$ in 5 Tagen, in der $\mathrm{NTZ}_{10}$ bereits am ersten Tag beinahe vollständig aufgebraucht. Diese starke $\mathrm{PO}_{4}$-Zehrung verhinderte auch bei wiederholter Düngung in der $\mathrm{NTZ}_{3 \times 7}$ eine Anreicherung von freien PhosphatIonen.

Erstmals konnte eine deutliche Beeinflussung der GOP-Komponenten durch die $\mathrm{PO}_{4}$-Düngung beobachtet werden (Abb. 15). Wichen die Konzentrationen in der $\mathrm{NTZ}_{10}$ und $\mathrm{NTZ}_{3 \times 7}$ nicht wesentlich von jenen der Vergleichszelle ab, so konnte in der $\mathrm{NTZ}_{\mathbf{3 0}}$ bis zum vierten Tag ein deutlicher Anstieg um rund $9 \mathrm{mg}$ GOP $/ \mathrm{m}^{3}$ festgestellt werden. Zu diesem Zeitpunkt lag die PP-Konzentration bei $37 \mathrm{mg} / \mathrm{m}^{3}$ und erreichte erst am achten Tag das Maximum von $42 \mathrm{mg} \mathrm{PP} / \mathrm{m}^{3}$. Im gleichen Zeitabschnitt verringerte sich aber die Phosphatkonzentration nur um $3 \mathrm{mg} / \mathrm{m}^{3}$. Stellt man zusätzlich die $2 \mathrm{mg}$ Sedimentationsverlust in Rechnung, so müssen $4 \mathrm{mg} \mathrm{GOP} / \mathrm{m}^{3}$ in $\mathrm{PP}$ über- 

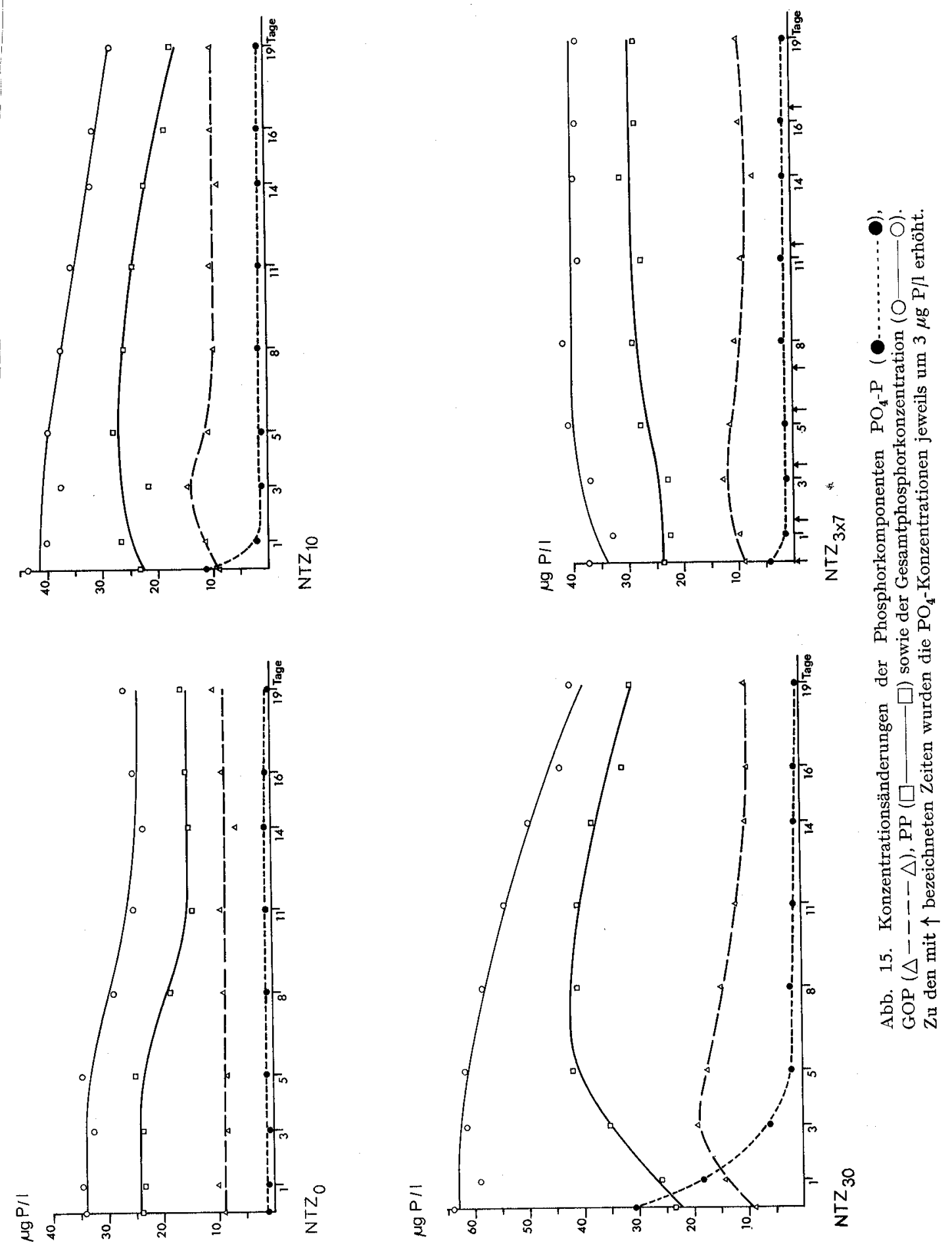
geführt worden sein. Damit konnte in unsern Versuchen erstmals beobachtet werden, dass durch Organismen ausgeschiedener GOP reassimiliert werden kann. Da über die Zusammensetzung der GOP-Komponente nichts bekannt ist, muss die Frage, weshalb die oft beträchtlichen Gehalte an GOP trotz P-Bedarf nicht inkorporiert werden, allerdings offen bleiben. Möglicherweise handelt es sich dabei um einen dynamischen Gleichgewichtszustand im System $\mathrm{PO}_{4}, \mathrm{GOP}, \mathrm{PP}$ oder aber um schwer abbaubare Phosphorverbindungen (s. S. 17).

\section{Photosyntheseaktivität}

In der Versuchsreihe $\mathrm{NTZ}_{0}, \mathrm{NTZ}_{\mathbf{1 0}}, \mathrm{NTZ}_{\mathbf{3 0}}$ wurden während der ganzen Versuchsdauer mit steigender Düngung steigende Assimilationsraten gemessen. Mit dem in der $\mathrm{NTZ}_{30}$ registrierten Maximalwert von $12,1 \mathrm{mg} \mathrm{C} / \mathrm{h} \cdot \mathrm{m}^{3}$ am elften Tag wurde der achtfache Wert der Kontrolle erreicht. Am gleichen Tag fiel die Assimilationsrate in der $\mathrm{NTZ}_{\mathbf{3} \times \mathbf{7}}$ nach nur insgesamt $15 \mathrm{mg} \mathrm{P} / \mathrm{m}^{3}$ Zugabe beinahe gleich hoch aus. Am vierzehnten, siebzehnten und neunzehnten Tag wurden die Werte von $\mathrm{NTZ}_{\mathbf{3}}$ durch diejenigen von $\mathrm{NTZ}_{\mathbf{3} \times \mathbf{7}}$ übertroffen, obwohl die maximale Düngung $\left(21 \mathrm{mg} / \mathrm{m}^{3}\right)$ stets unter derjenigen von $\mathrm{NTZ}_{\mathbf{3 0}}$ blieb. Daraus muss gefolgert werden, dass die Produktion

Tab. 23. Individuen pro Liter.

\begin{tabular}{|c|c|c|c|c|c|}
\hline NTZ & $\begin{array}{l}\text { Versuchstag } \\
\text { 1. Tag }\end{array}$ & 4. Tag & 8. Tag & 12. Tag & 15. Tag \\
\hline \multicolumn{6}{|c|}{ Oscillatoria rubescens } \\
\hline 0 & 17000 & 18000 & 21000 & 21000 & 21000 \\
\hline 5 & 17000 & 20000 & 22000 & 22000 & 20000 \\
\hline 10 & 18000 & 19000 & 17000 & 24000 & 19000 \\
\hline 30 & 16000 & 19000 & 20000 & 26000 & 23000 \\
\hline \multicolumn{6}{|c|}{ Stephanodiscus astraea } \\
\hline 0 & 53000 & 108000 & 191000 & 199000 & 250000 \\
\hline 5 & 50000 & 86000 & 161000 & 244000 & 308000 \\
\hline 10 & 66000 & 85000 & 195000 & 460000 & 293000 \\
\hline 30 & 60000 & 90000 & 251000 & 466000 & 297000 \\
\hline \multicolumn{6}{|c|}{ Stephanodiscus hantzschii } \\
\hline 0 & 167000 & 952000 & 2980000 & 4810000 & 7360000 \\
\hline 5 & 144000 & 831000 & 2742000 & 6160000 & 5180000 \\
\hline 10 & 261000 & 1079000 & 3692000 & 11770000 & 17500000 \\
\hline 30 & 222000 & 968000 & 4750000 & 9610000 & 13960000 \\
\hline \multicolumn{6}{|c|}{ Tabellaria fenestrata } \\
\hline 0 & 85000 & 123000 & 282000 & 291000 & 318000 \\
\hline 5 & 59000 & 76000 & 196000 & 450000 & 340000 \\
\hline 10 & 63000 & 110000 & 246000 & 533000 & 286000 \\
\hline 30 & 76000 & 124000 & 311000 & 733000 & 388000 \\
\hline \multicolumn{6}{|c|}{ Synedra cf. berolinensis } \\
\hline 0 & 34000 & 38000 & 153000 & 199000 & 498000 \\
\hline 5 & 30000 & 38000 & 73000 & 297000 & 582000 \\
\hline 10 & 54000 & 52000 & 157000 & 584000 & 780000 \\
\hline 30 & 62000 & 77000 & 133000 & 470000 & 780000 \\
\hline
\end{tabular}


nicht nur von der Gesamtmenge des zugefügten Nährstoffs abhängt, sondern entscheidend von der Zufuhr pro Zeiteinheit abhängt.

Qualitative und quantitative Planktonanalyse

Oscillatoria rubescens erreichte am zehnten Tag maximale Dichte, ohne eine eindeutige Reaktion auf die Phosphordüngung zu zeigen. Tabellaria fenestrata entwickelte sich in allen Zellen gut. In den gedüngten Proben übertrafen die Zellenzahlen pro Liter diejenigen der Kontrolle leicht, unterschieden sich aber untereinander nicht signifikant. Synedra spp. vermehrte sich in allen Zellen nur schwach, reagierte aber auf die Düngung eindeutig positiv. Maximale Dichte wurde am vierzehnten Tag in der NTZ ${ }_{3 \times 7}$ erreicht.

Synedra cf. berolinensis vermehrte sich bei fortgesetzter kleiner Düngung bis zum vierzehnten Tag eindeutig am besten. Durch die Initialdüngung von $10 \mathrm{mg} \mathrm{P} / \mathrm{m}^{3}$ konnte die Teilungsrate leicht gesteigert werden. Die stärkere Düngung in der $\mathrm{NTZ}_{\mathbf{3 0}}$ wirkte eher hemmend.

Stephanodiscus hantzschii hatte unter natürlichen Bedingungen im März bei relativ hoher $\mathrm{PO}_{4}$-Konzentration maximale Entfaltung erreicht. (Vgl. Experiment vom 2.3.67 bis 17.3.67.) Bei Versuchsbeginn trat diese Kieselalge in allen NTZ so selten auf, dass sie quantitativ nicht erfasst werden konnte. Im mittleren Versuchsabschnitt entwickelte sich Stephanodiscus hantzschii bei starker Düngung nochmals explosionsartig. Verglichen mit der Kontrollzelle wurde in der $\mathrm{NTZ}_{\mathbf{1 0}}$ am elften Versuchstag die 15 fache, in der $\mathrm{NTZ}_{30}$ gar die 50fache Individuendichte erreicht. Die wiederholte schwache Düngung stimulierte die Zellteilung nur unbedeutend.

Rhodomonas lacustris und $R h$. lens erreichten im ersten Drittel der Versuchsdauer maximale Entfaltung, ohne dass sich ein gesicherter Zusammenhang zwischen Düngung und Ertrag ableiten liess. Nach der Überschreitung des Maximums verringerte sich die Individuendichte der gedüngten Proben jedoch merklich langsamer. Die gleiche Verlustrate in allen vier Zellen vorausgesetzt, deutet das auf eine verstärkte Vermehrung in den gedüngten Proben hin, obwohl sich zu diesem Zeitpunkt die Phosphatkonzentrationen der vier Kulturen nicht mehr voneinander unterschieden. Wird eine antagonistische Wirkung der übrigen Algen ausgeschlossen, so weist diese Beobachtung darauf hin, dass Rh. lacustris und $R h$. lens Phosphor zu speichern vermögen. Die nicht näher identifizierten kugeligen grünen $\mu$-Algen von durchschnittlich etwa 1,8-2 $\mu \mathrm{m}$ Durchmesser reagierten auf die Düngung eindeutig mit gesteigerter Teilungsrate. Bereits am vierzehnten Versuchstag (nach insgesamt nur $18 \mathrm{mg} \mathrm{P} / \mathrm{m}^{3}$ Düngung) erreichten sie in der $\mathrm{NTZ}_{3 \times 7}$ die Individuendichte von $\mathrm{NTZ}_{30}$ und übertrafen sie in der Folge wesentlich. Diese Alge bewirkte wahrscheinlich in der $\mathrm{NTZ}_{3 \times 7}$ nach dem vierzehnten Versuchstag die überraschend hohen Assimilationsraten (vgl. Tab. 24).

Tab. 24. Photosyntheseraten (Angaben in $\mathrm{mg} \mathrm{C}_{\mathrm{ass}} / \mathrm{h} \cdot \mathrm{m}^{3}$ ).

\begin{tabular}{|c|c|c|c|c|c|c|c|c|}
\hline \multirow[t]{2}{*}{ NTZ } & \multicolumn{8}{|c|}{ Versuchstag } \\
\hline & 1. & 3. & 5. & 8. & 11. & 14. & 16. & 19. \\
\hline 0 & 2,52 & 2,17 & 3,01 & 1,76 & 1,68 & 1,13 & 1,26 & 0,85 \\
\hline 10 & 4,08 & 3,44 & 5,75 & 4,79 & 4,68 & 3,61 & 3,37 & 3,57 \\
\hline 30 & 4,99 & 4,85 & 7,60 & 10,11 & 12,80 & 7,42 & 7,79 & 7,33 \\
\hline $3 \times 7$ & 3,37 & 2,95 & 4,94 & 6,49 & 11,25 & 8,40 & 10,60 & 11,74 \\
\hline
\end{tabular}


Tab. 25. Individuen pro Liter.

\begin{tabular}{|c|c|c|c|c|c|}
\hline NTZ & $\begin{array}{l}\text { Versuchstag } \\
\text { 1. Tag }\end{array}$ & 5. Tag & 11. Tag & 14. Tag & 19. Tag \\
\hline \multicolumn{6}{|c|}{ Oscillatoria rubescens } \\
\hline 0 & 12000 & 14000 & 17000 & 13000 & 9000 \\
\hline $3 \times 7$ & 9000 & 12000 & 17000 & 11000 & 10000 \\
\hline 10 & 9000 & 13000 & 17000 & 12000 & 13000 \\
\hline 30 & 8000 & 10000 & 22000 & 13000 & 10000 \\
\hline \multicolumn{6}{|l|}{$\mu$-Algen } \\
\hline 0 & 16000000 & 32550000 & 23150000 & 21900000 & 16000000 \\
\hline $3 \times 7$ & 16000000 & 48000000 & 101000000 & 135000000 & 467000000 \\
\hline 10 & 13000000 & 33000000 & 54150000 & 58000000 & 19000000 \\
\hline 30 & 24000000 & 39000000 & 150000000 & 160000000 & 120000000 \\
\hline \multicolumn{6}{|c|}{ Stephanodiscus hantzschii } \\
\hline 0 & - & - & 101000 & vereinzelt & vereinzelt \\
\hline $3 \times 7$ & - & - & 443000 & 687000 & vereinzelt \\
\hline 10 & - & - & 1501000 & vereinzelt & vereinzelt \\
\hline 30 & - & - & 5150000 & 6450000 & 3630000 \\
\hline \multicolumn{6}{|c|}{ Tabellaria fenestrata } \\
\hline 0 & 169000 & 499000 & 567000 & 541000 & 783000 \\
\hline $3 \times 7$ & 156000 & 451000 & 1162000 & 1060000 & 1008000 \\
\hline 10 & 152000 & 383000 & 894000 & 1010000 & 1300000 \\
\hline 30 & 145000 & 291000 & 858000 & 1046000 & 945000 \\
\hline \multicolumn{6}{|c|}{ Synedra spp. } \\
\hline 0 & 13000 & 19000 & 20000 & 15000 & 8000 \\
\hline $3 \times 7$ & 10000 & 16000 & 42000 & 46000 & 21000 \\
\hline 10 & 11000 & 20000 & 33000 & 34000 & 22000 \\
\hline 30 & 13000 & 16000 & 29000 & 40000 & 37000 \\
\hline \multicolumn{6}{|c|}{ Synedra cf. berolinensis } \\
\hline 0 & 69000 & 100000 & 141000 & 296000 & 929000 \\
\hline $3 \times 7$ & 71.000 & 102000 & 626000 & 757000 & 586000 \\
\hline 10 & 57000 & 129000 & 374000 & 384000 & 535000 \\
\hline 30 & 73000 & 87000 & 222000 & 172000 & 542000 \\
\hline \multicolumn{6}{|c|}{ Rhodomonas lacustris und Rhodomonas lens } \\
\hline 0 & 1440000 & 3340000 & 1110000 & 686000 & 568000 \\
\hline $3 \times 7$ & 1098000 & 2525000 & 1110000 & 1320000 & 626000 \\
\hline 10 & 1278000 & 3800000 & 1432000 & 1140000 & 879000 \\
\hline 30 & 1224000 & 4010000 & 3440000 & 2740000 & 1372000 \\
\hline
\end{tabular}

\subsection{Versuch vom 28.6.67 bis 12.7.67}

Die Versuchsanordnung stimmte mit jener des Versuchs von Abschnitt 4.5 überein. Eine Nährstofftestzelle enthielt unverändertes Seewasser, in zwei weiteren wurde der Gesamtphosphorgehalt durch eine einmalige Phosphatzugabe um 10 bzw. $30 \mathrm{mg} / \mathrm{m}^{3}$ erhöht, und der vierten Zelle wurden während der Versuchsdauer von 15 Tagen in sieben Raten je $3 \mathrm{mg} \mathrm{PO}_{4}-\mathrm{P} / \mathrm{m}^{3}$ also insgesamt $21 \mathrm{mg} \mathrm{P} / \mathrm{m}^{3}$ zugeführt. 
Zeitliche Veränderung der Phosphorkomponenten $\left(\mathrm{PO}_{4}, \mathrm{GOP}, \mathrm{PP}\right)$

Das zugesetzte Phosphat wurde anfänglich mit der gleichen Geschwindigkeit, später aber wesentlich langsamer aufgezehrt als im vorangehenden Versuch, so dass die Phosphatkonzentration in der $\mathrm{NTZ}_{30}$ erst nach 13 Tagen wieder auf die ursprüngliche Konzentration absank (Abb. 16). In der $\mathrm{NTZ}_{3 \times 7}$ konnte trotz wiederholter Düngung nie eine Erhöhung des freien Phosphats festgestellt werden.

Bei Versuchsbeginn bewirkte die $\mathrm{PO}_{4}$-Düngung in der $\mathrm{NTZ}_{10}$ und vor allem in der $\mathrm{NTZ}_{30}$ eine eindeutige Erhöhung der GOP-Konzentration, die am ersten Versuchstag mit einem ersten Maximum von $14,9 \mathrm{mg} \mathrm{P} / \mathrm{m}^{3}$ beinahe doppelt so hoch ausfiel wie in der Vergleichsprobe. Nach dem vierten Tag erhöhte sich die GOP-Konzentration in allen vier Zellen leicht, um nach dem zehnten Tag wieder abzufallen.

Durch die $\mathrm{PO}_{4}$-Düngung wurde die PP-Konzentration in der $\mathrm{NTZ}_{10}$ im Maximum um $9 \mathrm{mg} / \mathrm{m}^{3}$, in der $\mathrm{NTZ}_{30}$ um $22 \mathrm{mg} / \mathrm{m}^{3}$ und in der $N T Z_{3 \times 7}$ um $11 \mathrm{mg} / \mathrm{m}^{3}$ erhöht. Auffallend ist, dass die Zunahmen an PP am ersten Versuchstag in den $\mathrm{NTZ}_{\mathbf{1 0}}$ und $\mathrm{NTZ}_{30}$ praktisch gleich hoch ausfielen, obwohl die $\mathrm{PO}_{4}$-Abnahme um rund $7 \mathrm{mg} \mathrm{P} / \mathrm{m}^{3}$ differierten. Diese Differenz wurde jedoch wie erwähnt als GOP wiedergefunden.

\section{Photosyntheseaktivität}

Steigende Düngermenge hatte eine deutliche Steigerung der Photosynthese zur Folge. In den ersten vier Versuchstagen lagen die Photosyntheseraten der $\mathrm{NTZ}_{10}$ und $\mathrm{NTZ}_{3 \times 7}$ etwa 1,5-2mal so hoch wie jene der Vergleichszelle. Die Kohlenstoffassimilation der Proben aus der $\mathrm{NTZ}_{\mathbf{3 0}}$ fiel in diesem Zeitabschnitt etwas niedriger aus als diejenige der schwächer gedüngten Zellen. Während sich die Aktivität der NTZ nach dem vierten Tag mehr und mehr jener der Vergleichszelle anglich, steigerte sich die Wirkung der Düngung in den beiden übrigen Zellen bis zum zehnten Tag. Zu diesem Zeitpunkt wurde in der $\mathrm{NTZ}_{30}$ rund 6,5mal, in der $\mathrm{NTZ}_{3 \times 7}$ nach insgesamt $15 \mathrm{mg} \mathrm{P} / \mathrm{m}^{3}$ zugeführtem $\mathrm{PO}_{4}$ rund 3 mal soviel Kohlenstoff assimiliert wie in der Vergleichszelle. Stellt man die Steigerung der Kohlenstoffassimilation der zugegebenen Phosphatmenge gegenüber, ergibt sich, dass das zugeführte Phosphat in der $\mathrm{NTZ}_{3 \times 8}$ während der ersten 4 Tage am besten, während der restlichen Versuchsdauer besser als in der $\mathrm{NTZ}_{10}$ und zum mindesten gleich gut wie in der $\mathrm{NTZ}_{30}$ ausgenützt wurde.

\section{Qualitative und quantitative Planktonanalysen}

Die bereits im letzten Versuch erwähnten kleinen kugeligen Grünalgen ( $\mu$-Algen) dominierten diesmal das planktische Bild eindeutig. Sie reagierten auf die Phosphatdüngung mit stark gesteigerter Teilungsrate und erreichten am zehnten Tag in der $\mathrm{NTZ}_{\mathbf{3 0}}$ eine maximale Dichte von 790000000 Individuen pro Liter, was dem rund 600 fachen Ausgangswert entsprach.

Wie aus der Tab. 27 ersichtlich ist, zeigte ausser Dictyosphaerium keine Form des Netzplanktons eine positive Reaktion auf die Phosphatdüngung, und in den meisten Fällen überwog die Verlustrate die Zuwachsrate.

Dinobryon spp. entwickelte sich in der Vergleichszelle und $\mathrm{NTZ}_{\mathbf{3} \times \mathbf{7}}$ wesentlich besser als in der $\mathrm{NTZ}_{\mathbf{1 0}}$ und $\mathrm{NTZ}_{\mathbf{3 0}}$. Diese Beobachtung bestätigt die Ergebnisse von RODHE [45], wonach sich $D$. divergens im schwachgedüngten Wasser des Erkensees 

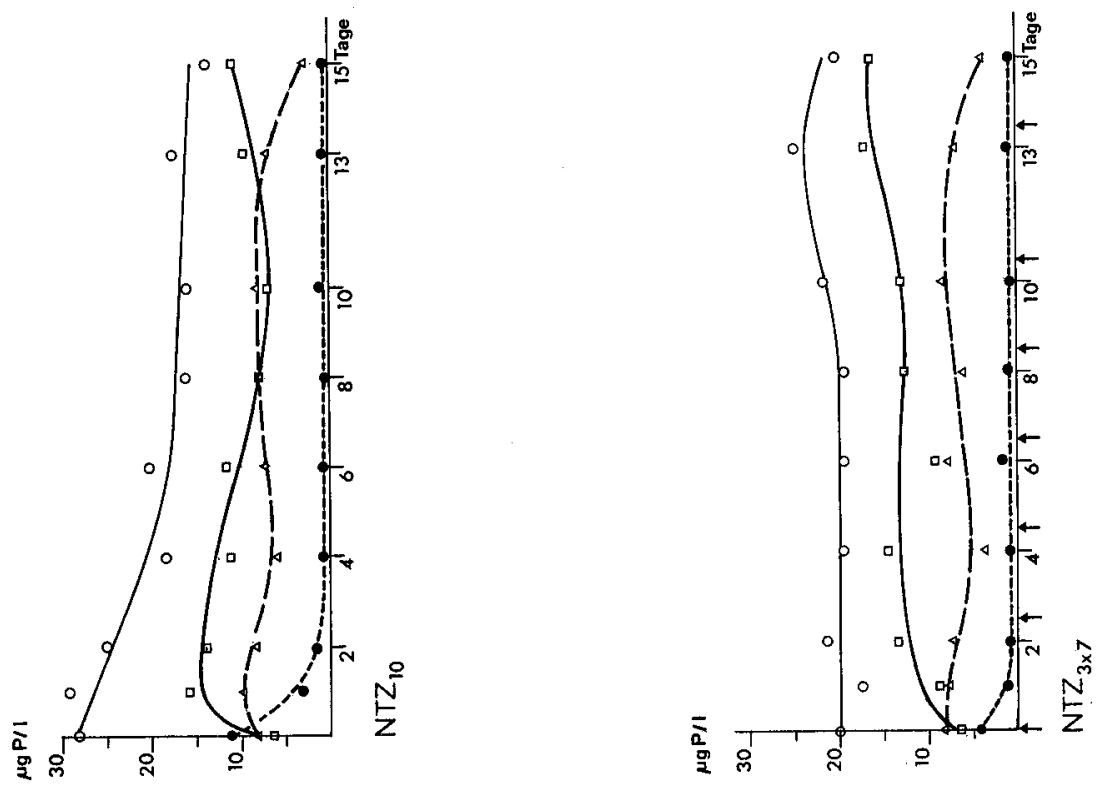

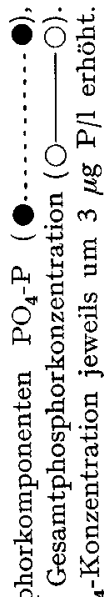
嵌
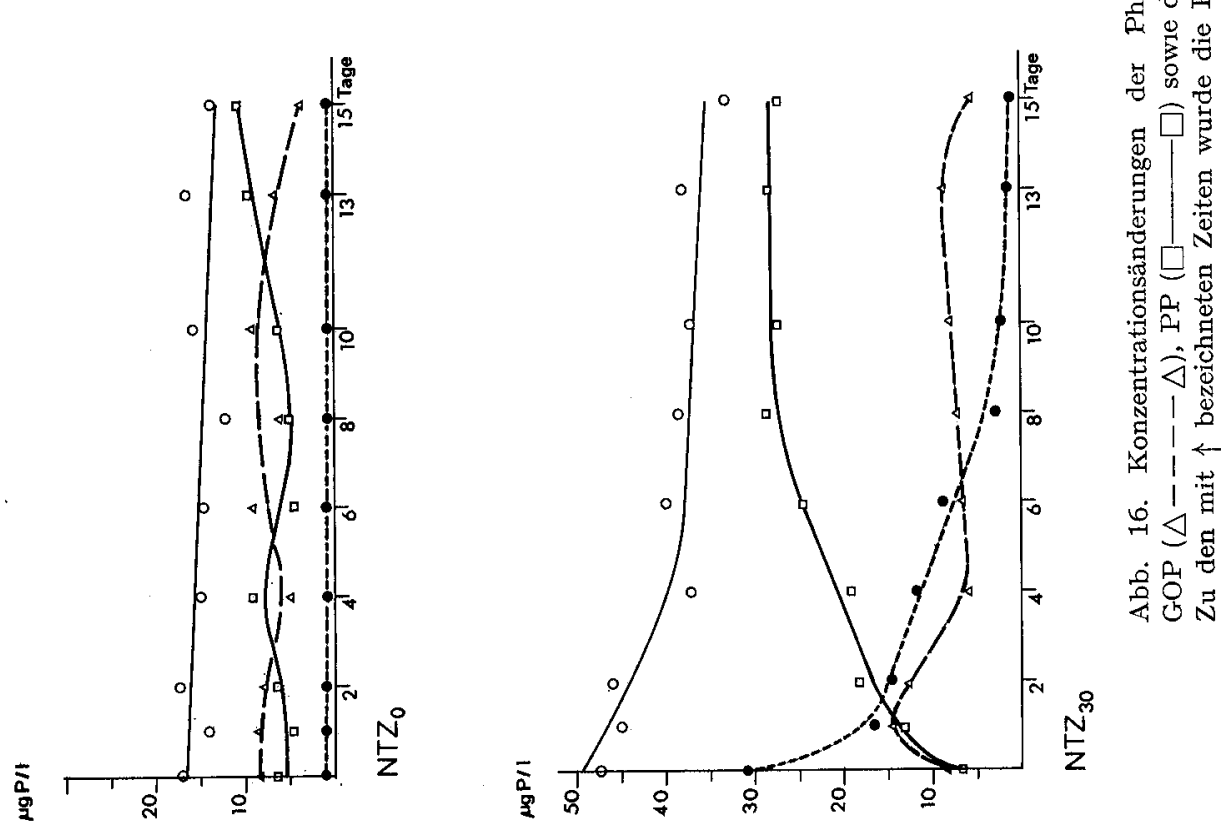
Tab. 26. Photosyntheseraten (Angaben in $\mathrm{mg} \mathrm{C} \mathrm{C}_{\mathbf{a s s}} / \mathrm{h} \cdot \mathrm{m}^{3}$ ).

\begin{tabular}{|c|c|c|c|c|c|c|c|c|}
\hline \multirow[t]{2}{*}{ NTZ } & \multicolumn{8}{|c|}{ Versuchstag } \\
\hline & 1. & 2. & 4. & 6. & 8. & 10. & 13. & 15. \\
\hline 0 & 4,16 & 3,17 & 2,32 & 2,11 & 2,32 & 3,72 & 2,48 & 3,14 \\
\hline 10 & 5,25 & 5,76 & 4,91 & 4,72 & 4,75 & 3,98 & 2,52 & 3,32 \\
\hline 30 & 6,32 & 4,14 & 4,72 & 8,97 & 20,52 & 22,70 & 6,31 & 5,15 \\
\hline $3 \times 7$ & 6,68 & 5,75 & 4,94 & 6,91 & 10,59 & 11,50 & 4,69 & 7,95 \\
\hline
\end{tabular}

Tab. 27. Individuen pro Liter.

\begin{tabular}{llrrr}
\hline NTZ & $\begin{array}{l}\text { Versuchstag } \\
1 . \text { Tag }\end{array}$ & 6. Tag & 10. Tag & 15. Tag \\
\hline$\mu$-Algen & & & & \\
0 & 1391000 & 6580000 & 12780000 & 13900000 \\
$3 \times 7$ & 2122000 & 47750000 & 116800000 & 18000000 \\
10 & 2538000 & 16400000 & 13200000 & 10600000 \\
30 & 1300000 & 86800000 & 790000000 & 40800000
\end{tabular}

Dictyosphaerium Ehrb.

0
$3 \times 7$
10
30

9750
34000
10000
13000

$\begin{array}{rr}8000 & 8700 \\ 40000 & 28000 \\ 16000 & 9200 \\ 15000 & 17000\end{array}$

Dinobryon spp.

$\begin{array}{lr}0 & 9000 \\ 3 \times 7 & 10000 \\ 10 & 10000 \\ 30 & 9000\end{array}$

34000
57000
23000
15000

62000

51000

$37000 \quad 46000$

$29000 \quad 24000$

$10000 \quad 5000$

Tabellaria fenestrata

$\begin{array}{ll}0 & 446000 \\ 3 \times 7 & 414000 \\ 10 & 524000 \\ 30 & 504000\end{array}$

342000

416000

380000

345000

256000

334000

231000

73000

249000

298000

109000

12000

Fragilaria crotonensis

$\begin{array}{lr}0 & 10000 \\ 3 \times 7 & 9000 \\ 10 & 10000 \\ 30 & 11000\end{array}$

7000
10000
9000
10000

11000

15000

10000

3000

7400

12000

3000

9000

Cryptomas spp.

\begin{tabular}{lr}
0 & 282000 \\
$3 \times 7$ & 93000 \\
10 & 320000 \\
30 & 494900 \\
\hline
\end{tabular}

22000

47000

47000

25000

34000

29000

30000

15000

5000

7000 
(Zugabe kleiner als $4 \mu \mathrm{g} \mathrm{PO}_{4}-\mathrm{P} / \mathrm{l}$ ) manchmal besser, im stärker gedüngten Wasser (mehr als $5 \mu \mathrm{g} \mathrm{PO}_{4}-\mathrm{P} / 1$ ) immer schlechter als im unbehandelten Wasser entwickelte.

Eine Hemmung des Wachstums durch antagonistische Wirkung anderer Algen kann in diesem Fall mit grosser Wahrscheinlichkeit ausgeschlossen werden, da die Zellzahlen des Netzplanktons in den verschiedenen NTZ nur unbedeutend voneinander abwichen, und Dinobryon spp. sich in der $\mathrm{NTZ}_{3 \times 7}$ trotz wesentlich höherer $\mu$-Algen-Konzentration eindeutig besser entwickelte als in der $\mathrm{NTZ}_{\mathbf{1 0}}$.

\section{Diskussion}

\subsection{Phosphataufnahme}

Das zugesetzte Phosphat wurde zu den verschiedenen Jahreszeiten vom Phytoplankton unterschiedlich rasch inkorporiert.

Im Zeitabschnitt Mai bis Oktober (Experimente von Abschnitt 4.1, 4.2, 4.5, 4.6) war die Aufnahmegeschwindigkeit bei Versuchsbeginn maximal, und es stellte sich nach wenigen Tagen bereits ein neues $\mathrm{PO}_{4}$-PP-Gleichgewicht ein. Das Phosphat wurde dabei, von einer Ausnahme abgesehen $\left(\mathrm{NTZ}_{\mathbf{3 0}}\right.$ in Abschnitt 4.2), stets bis auf die natürliche ursprüngliche Konzentration aufgezehrt. Die Aufnahmegeschwindigkeit zur Zeit $t=0$ war abhängig von der Phosphatanfangskonzentration. In einigen Fällen schien die Aufnahmegeschwindigkeit direkt proportional der Anfangskonzentration zu sein. Dieses Ergebnis deckt sich mit den Befunden von Keтchum [68]. Er zeigte an Nitzschia closterium, dass die Aufnahmeraten bis zu einer bestimmten Anfangskonzentration dieser direkt proportional sind und dass bei höhern Anfangskonzentrationen ein Sättigungsplateau der Aufnahmegeschwindigkeit erreicht wird. Die $\mathrm{PO}_{4}$-Aufnahmegeschwindigkeit ist ferner abhängig von der Nitratkonzentration. $\mathrm{Zu}$ analogen Resultaten kamen auch Ramamurthy und KrIshnamurTHY, die mit Trichodesmium erytraeum arbeiteten [69].

Die Veränderung der PP-Konzentration im März 1966 erinnert stark an eine typische Wachstumskurve. Die Aufnahmegeschwindigkeit zur Zeit $t=0$ war minimal (Anlaufphase), stieg im mittleren Versuchsabschnitt merklich an (logarithmische Phase) und erreichte wieder Minimalwerte (stationäre Phase). Die starke Zunahme an PP im mittleren Versuchsabschnitt fiel zeitlich mit deutlich erhöhten Photosynthese- und Teilungsraten zusammen.

Im Winter war die Überführung von $\mathrm{PO}_{4}-\mathrm{P}$ in $\mathrm{PP}$ unbedeutend und konnte kaum gesichert nachgewiesen werden.

Es können daraus folgende Schlüsse gezogen werden:

Sommerplankton (Mai bis Oktober): Hoher P-Bedarf, grosse Speicherkapazität.

Winterplankton (Dezember/Januar): Kein P-Bedarf, keine Speicherkapazität.

Frühjahrsplankton (März): P-Bedarf, aber keine oder nurgeringe Speicherkapazität.

Bei der Interpretation der Resultate muss man sich aber bewusst sein, dass der gefundene PP nicht unbesehen dem organismisch fixierten Phosphor gleichgesetzt werden darf. Ein Teil davon ist mit Detritus assoziiert, und es ist anzunehmen, dass Detritus auch $\mathrm{PO}_{4} \mathrm{zu}$ adsorbieren vermag und daher eine aktive Phosphataufnahme 
durch das Phytoplankton vortäuschen kann. Es wurde jedoch von andern Autoren (z.B. $[14,17,45,79])$ unter günstigeren Versuchsbedingungen schon vielfach nachgewiesen, dass an Phosphor verarmte Algen bei Phosphatangebot ihr P-Defizit in kurzer Zeit zu decken und darüber hinaus noch Phosphor zu speichern vermögen. Die Wahrscheinlichkeit ist daher gross, dass auch in unseren Experimenten die rasche Zunahme an PP ohne entsprechende Zellvermehrung auf die Speicherung von PP zurückzuführen ist.

\subsection{Sedimentationsverluste}

Bei den Versuchen fällt auf, dass die Verlustraten des Gesamtphosphors in den gedüngten Proben oft höher sind als in den Vergleichszellen. Dies bedeutet, dass sich die Gesamtphosphorkonzentrationen bei genügend langer Versuchsdauer wieder dem Ausgangswert angleichen würden. Im folgenden wird versucht, diese Erscheinung anhand eines Modells zu erklären. Es ist bekannt, dass das Phytoplankton bei einem Phosphorüberangebot Phosphor zu speichern vermag $[14,17,45,79]$. Wenn sich nach vollständiger Aufzehrung des Phosphats aus dem Nährmedium die Algen weiterhin teilen, so muss sich der P-Gehalt der Zellen wieder verringern. Da der Gesamtphosphorgehalt der gesamten Biomasse konstant bleibt, so gilt folgende Gleichung:

$$
c_{\mathbf{0}} N_{\mathbf{0}}=\text { konstant }=c_{t} N_{t}
$$

Dabei bedeutet $c_{0}$ den P-Gehalt der Algenzellen und $N_{\mathbf{0}}$ die Anzahl der Individuen nach Abschluss der $\mathrm{PO}_{4}$-Aufnahme. $c_{t}$ und $N_{t}$ geben den P-Gehalt pro Zelle und die Individuenzahl zu einem späteren Zeitpunkt $t$ an.

Die Zellteilung erfolge in einem begrenzten Zeitabschnitt exponentiell nach der Zinseszinsformel:

$$
N_{t}=N_{0}(1+0,01 p)^{t}
$$

$$
p=\text { täglicher Zuwachs in Prozent ausgedrückt. }
$$

Aus (1) und (2) lässt sich der P-Gehalt pro Algenzelle zur Zeit $t$ berechnen:

$$
c_{t}=\frac{c_{0} N_{0}}{N_{t}}=\frac{c_{0}}{(1+0,01 p)^{t}}
$$

In unserm Experiment wurde aber die Individuenzahl nicht nur täglich mit $p \%$ ver-

\begin{tabular}{|c|c|c|}
\hline Zeit & $n$ & $=$ Anzahl im Wasser schwebende Algen \\
\hline$t=0$ & $n_{0}$ & $=N_{0}$ \\
\hline$t=1$ & $n_{1}$ & 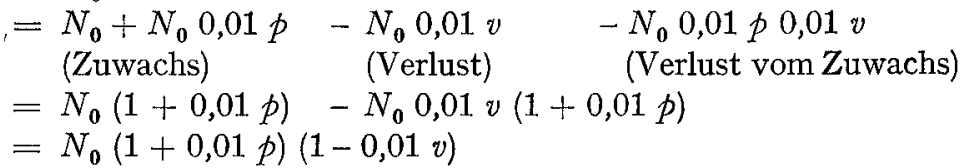 \\
\hline$t=2$ & $n_{2}$ & $\begin{array}{l}=n_{1}+n_{1} 0,01 p-n_{1} 0,01 v-n_{1} 0,01 p 0,01 v \\
=n_{1}(1+0,01 p)-(1-0,01 v) \\
=N_{0}(1+0,01 p)^{2}(1-0,01 v)^{2}\end{array}$ \\
\hline$t=t$ & $n_{t}$ & $=N_{0}(1+0,01 p)^{t}(1-0,01 v)^{t}$ \\
\hline
\end{tabular}
mehrt, sondern ebenso durch die Sedimentationsverluste täglich wieder um $v \%$ verringert. $\mathrm{N}$ gibt an, wie viele Zellen im Kulturgefäss total vorhanden sind, $n$ wie viele Individuen noch frei im Wasser schweben (noch nicht sedimentiert sind). 
Aus dem bekannten P-Gehalt pro Algenzelle (3) und der zur Zeit $t$ noch schwebenden Algenzellen lässt sich der noch schwebende PP berechnen.

$$
\mathrm{PP}_{\text {schweb }(t)}=c_{t} n_{t}=\frac{c_{0}}{(1+0,01 p)^{t}} N_{0}(1+0,01 p)^{t}(1-0,01 v)^{t}
$$

Der Sedimentationsverlust im Zeitabschnitt «0 bis $t »$ ergibt sich aus

$$
\begin{aligned}
\text { Sedimentationsverlust an } \mathrm{PP} & =\mathrm{PP}_{\text {schweb }(0)}-\mathrm{PP}_{\text {schweb }(t)} \\
& =c_{\mathbf{0}} N_{\mathbf{0}}-c_{\mathbf{0}} N_{\mathbf{0}}(\mathbf{1}-0,01 v)^{t}
\end{aligned}
$$

Sedimentationsverlust an $\mathrm{PP}=c_{0} N_{0}\left(1-(1-0,01 v)^{t}\right.$

Da wir nicht mit Monokulturen arbeiteten, setzt sich die Grösse $N$ aus einer bestimmten Anzahl Partikeln verschiedenster Art zusammen (verschiedene Algen und verschiedene Formen von Detritus). Jeder Algenspezies ist eine bestimmte Verlustrate eigen, und sie reagiert auf die Phosphordüngung artspezifisch, sowohl was Speicherungsvermögen als auch was die Teilungsrate betrifft. In unserm Experiment liegen die Verhältnisse also derart kompliziert, dass sie mathematisch nicht genügend erfasst werden können. Das ändert aber nichts an der qualitativen Aussage der Gleichung (5): Wenn die tägliche Sedimentationsrate v, die vom P-Gehalt der Algenzellen unabhängig ist, grösser als Null ist, so ist der Verlust an PP um so grösser, je mehr Phosphor gespeichert wurde, und zwar unabhängig von der Teilungsrate. Da diese Überlegungen natürlich auch im See ihre Gültigkeit behalten, bedeutet das, dass jede phosphorspeichernde Alge die mögliche Produktion eines Gewässers momentan vermindert.

Unsere Ergebnisse stimmen in dieser Beziehung deutlich mit jenen von EINSELE [8] überein, die zeigten, dass zur trophogenen Schicht zugefügtes Phosphat im Seston rasch inkorporiert und durch Sedimentation aus der trophogenen Schicht ausgeschieden wird. FINDENEGG [70] weist darauf hin, dass Oscillatoria rubescens im Frühjahr der oberen trophogenen Schicht Phosphat entzieht, sich im Sommer in tiefere Schichten zurückzieht und daher die Produktion in den höhern Wasserschichten im Sommer verringert. Nach GRIM [17] können vor allem Diatomeen dank ihrem hohen P-Speicherungsvermögen und ihren hohen Sedimentationsraten wesentlich zur Phosphorverarmung der trophogenen Schicht beitragen. Er bezeichnet diesen Vorgang direkt als «natürliche dritte Reinigungsstufe». Sicher trägt sie zusammen mit der P-Falle "Sediment» wesentlich dazu bei, dass sich die dauernde humanaktive Belastung der Gewässer in den letzten Jahren nicht noch verheerender ausgewirkt hat. Die trotzdem rasch fortschreitende Produktionszunahme (z. B. gemessen an der hypolimnischen Sauerstoffzehrung) zeigt jedoch, dass der Eliminationseffekt dieser «natürlichen dritten Reinigungsstufe» nicht ausreicht, um die dauernde Nährstoffzufuhr schadlos aufzufangen.

\subsection{Einfluss der kïnstlich erhöhten Gesamtphosphorkonzentration auf die Entwicklung einzelner Phytoplankter}

Die Experimente von RodHe [45] zeigten, dass der Phosphorbedarf der verschiedenen planktischen Algen so stark variiert, dass die gleiche Phosphatkonzentration für das Wachstum einer ersten Alge suboptimal (begrenzend), für jenes einer zweiten optimal und für dasjenige einer dritten sogar superoptimal (hemmend) 
wirken kann. Er teilte die verschiedenen Arten je nach ihrem Phosphorbedarf in die folgenden drei Gruppen ein:

I. Algen, deren ganzer optimaler Bereich unterhalb $20 \mu \mathrm{g} \mathrm{P} / 1$ liegt.

II. Algen, die sich zwar bereits unterhalb $20 \mu \mathrm{g} \mathrm{P} / 1$, aber auch bei höheren Konzentrationen optimal entwickeln.

III. Algen, deren ganzer optimaler Bereich oberhalb $20 \mu \mathrm{g}$ P/l liegt.

$\mathrm{Zu}$ den Algen mit sehr geringem Phosphorbedarf (Gruppe I) gehören Dinobryon divergens und Uroglena americana (Chrysophyceae). In der Gruppe II ordnete er Asterionella formosa als Vertreter der Kieselalgen ein, während in der dritten Gruppe die Grünalgen wie Scenedesmus quadricauda zu finden sind. In dieser Gruppe ist nach Untersuchungen von STAUB [71] auch Oscillatoria rubescens und nach GERLOFF und Skoog [72] auch Microcystis aeruginosa einzureihen. PAvoni [73] kam aufgrund sehr umfangreicher Felduntersuchungen zu einer auffallend ähnlichen Klassifizierung:

\begin{tabular}{lll}
\hline Seentyp & $\begin{array}{l}\text { Häufigste Algengruppen } \\
\text { nach PAvoni }\end{array}$ & $\begin{array}{l}\text { Gruppierung nach } \\
\text { RoDHe }\end{array}$ \\
\hline Oligotroph & Chrysophyceae & Gruppe I \\
& Diatomeae & Gruppe II \\
Mesotroph & Diatomeae & Gruppe II \\
& Chrysophyceae & Gruppe I \\
& Cyanophyta & Gruppe III (STAUB) \\
Eutroph & Cyanophyta & Gruppe III (STAUB) \\
& Diatomeae & Gruppe II \\
& Chlorophyceae & Gruppe III \\
\hline
\end{tabular}

In Tab. 28 wurden die Reaktionen jener Algen, die in den Experimenten am häufigsten auftraten, nochmals zusammengestellt. Dabei wurde die Individuendichte der Vergleichszelle $\left(\mathrm{NTZ}_{0}\right) I_{0}=1$ gesetzt und jene der gedüngten Proben als Vielfaches davon auf halbe Einheiten gerundet angegeben. Als Stichtag wurde jener Versuchstag gewählt, an welchem in einer der vier Nährstofftestzellen die höchste während des Versuchs registrierte Dichte beobachtet wurde. Ausgehend von der Annahme, dass eine Alge um so stärker auf die Phosphatdüngung reagiere, je weiter die optimalen Nährstoffverhältnisse von den gegebenen natürlichen abweichen, wurden die Resultate von RODHE [45] durchwegs bestätigt. Die Grünalgen reagierten ausnahmslos mit stark erhöhtem, z.T. explosionsartigem Wachstum. Es zeigte sich ferner, dass die natürlichen Phosphorkonzentrationen im See für die meisten beobachteten Diatomeen suboptimal, für Dinobryon spp. aber superoptimal sind. Maximale Reaktion unter Diatomeen zeigte Stephanodiscus hantzschii, eine in eutrophen Gewässern zu Massenentfaltungen neigende Form. Oscillatoria rubescens, bekannt als Indikator für eutrophierte Gewässer, reagierte kaum auf die Phosphatdüngung. Die hohen Relativwerte vom August 1966 werden hauptsächlich dadurch bedingt, dass sich diese Alge in der Vergleichszelle $\left(\mathrm{NTZ}_{0}\right)$ so schlecht entwickelte, dass die Verlustraten die Zuwachsraten übertrafen; sie zeigen aber dennoch, dass optimale und natürliche P-Konzentration sehr nahe beieinander liegen. Nach STAUB [71] wird das Wachstum von Oscillatoria rubescens begrenzt, wenn der prozentuale Anteil des Gesamtphosphors am 
Tab. 28. Relative Individuendichten in Abhängigkeit von der Gesamtphosphorkonzentration. Bezogen auf die Individuendichte $I_{0}=1$ der Kontrollzelle $\left({ }^{*} \mathrm{NTZ}_{3} \times{ }_{7}\right)$.

Die Ziffern im Tabellenkopf beziehen sich auf die durchschnittliche Ges.-P-Konz. ( $\mu \mathrm{g} \mathrm{P} / \mathrm{l})$.
August 1966

$1.7,5 \quad 23,8 \quad 31,5 \quad 47,0$
Oktober 1966

$20,5 \quad 31,5 \quad 51,5$

\section{CYANOPHYTA}

Gomphosphaeria lacustris Chodat . . . . . . . . Oscillatoria rubescens D.C. . . . . . . . . . . .

$\begin{array}{lll}\overline{7}, 5 & - & 10\end{array}$

$\begin{array}{lll}1 & 1,5 & 0,5 \\ 1 & 1,5 & 1,5\end{array}$

\section{CHLOROPHYTA}

Gemellicystis neglecta Teiling em. Skuja . . . . . $\mu$-Algen . Nephrocytium agardhianum Naeg.. . . . . . . . 1

Dictyosphaerium pulchellum Wood . . . . . . . -

Ulothrix amphigranulata Skuja. . . . . . . . . 1

Mongeotia sp.

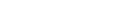

\section{CHRYSOPHYTA}

Chrysophyceae

Dinobryon spp.

Diatomeae

Stephanodiscus astraea (Ehrnb.) Grun. . . . . . . -

Stephanodiscus hantzschii Grun.

Stephanodiscus spp. und Cyclotella spp. . . . . .

Tabellaria fenestrata (Lyngb.) Kg. . . . . . . . -

Fragilaria crotonensis (Edw.) Kitt. . . . . . . . -

Asterionella formosa Hassall . . . . . . . . . . -

Synedra spp. . . . . . . . . . . . . . . . . . -

Synedra cf. berolinensis

\section{PYRROPHYTA}

Cryptophyceae

Rhodomonas lacustris Pascher et Ruttner

Rhodomonas lens Pascher et Ruttner

Cryptomonas ovata Ehrnb.

Cryptomonas erosa Ehrnb.

Trockengewicht unter 0,3\% fällt (kritische Konzentration im Sinne von GERLOFF und Skoog [72]). Direkte Messungen von Pavoni [74] haben ergeben, dass für Oscillatoria rubescens dieser Wert im Vierwaldstättersee stets höher als 0,3\% liegt und im Maximum sogar 2\% überschreiten kann. Werte, die STAuB im Zürichsee und Burgäschisee ermittelte, schwanken zwischen 0,3 und $0,5 \% \mathrm{P}$, bezogen auf das Trockengewicht. Diese Luxuswerte [45] erklären, warum sich eine Erhöhung der Phosphatkonzentration im allgemeinen kaum auf das Wachstum dieser Algen auswirkte.

In rasch wachsenden Kulturen wurden solche Luxuswerte aber erst erreicht, wenn Anfangskonzentrationen von mehr als $3 \mathrm{mg} \mathrm{PO}_{4}-\mathrm{P} / 1$ vorgelegt wurden, also bei 


$\begin{array}{lllllllllllllll}- & - & - & - & - & - & - & - & - & - & - & - & - & - & - \\ 1,5 & 1 & 1,5 & 1 & 1 & 1 & 1 & 1 & 1 & 1 & 1 & - & - & - & - \\ & & & & & & & & & & & & & & \\ - & - & - & - & - & - & - & - & - & - & - & 1 & 2 & 1 & 3 \\ - & - & - & - & - & - & - & 1 & 1 & 30 & 8 & 1 & 1 & 10 & 61 \\ - & - & - & - & - & - & - & - & - & - & - & - & - & - & - \\ - & - & - & - & - & - & - & - & - & - & - & 1 & 2 & 5 & 2 \\ - & - & - & - & - & - & - & - & - & - & - & - & - & - & - \\ - & - & - & - & - & - & - & - & - & - & - & - & - & - & -\end{array}$

$\begin{array}{lll}- & - & - \\ - & - & - \\ 1 & 0,5 & 1 \\ 1 & 0,5 & 0,5 \\ - & - & - \\ 0,5 & 0,5 & 0,5 \\ - & - & - \\ 1 & 1 & 1\end{array}$

$\begin{array}{llll}1 & \mathbf{1}, 5 & 2 & 2,5 \\ 1 & 1 & 2,5 & 2 \\ - & - & - & - \\ 1 & 1,5 & 2 & 2,5 \\ - & - & - & - \\ - & -- & - & - \\ - & -- & - & - \\ 1 & 1 . & 1,5 & 1,5\end{array}$

$\begin{array}{llll}- & - & - & - \\ - & - & - & - \\ 1 & 14 & 4,5 & 51 \\ - & - & - & - \\ 1 & 1,5 & 1,5 & 1 \\ - & - & - & - \\ - & - & - & - \\ 1 & 2 & 3 & 2,5 \\ 1 & 1 & 2,5 & 0,5\end{array}$

Konzentrationen, die 100-1000mal höher liegen, als sie im Vierwaldstättersee je erreicht werden. Es wäre denkbar, dass diese Alge unter den hoch suboptimalen Nährstoffverhältnissen am natürlichen Standort zwar in der Lage ist, in genügendem Mass Phosphat aufzunehmen, dass ihr aber andere wichtige Faktoren, die das Wachstum und damit die Verdünnung des zelleigenen Phosphorgehalts erlauben würden, fehlen. Nach VolLENWEIdER [7j] ist dabei vor allem an Stickstoffverbindungen zu denken. RODHE [45] und MACKERETH [14] haben für Asterionella formosa gezeigt, dass Phosphat bei der gleichen Anfangskonzentration aus natürlichem Seewasser besser aufgenommen werden kann als aus synthetischen Nährlösungen. Es wäre zu prüfen, ob diese Beobachtung auch auf Oscillatoria rubescens übertragen werden kann. 
5.4 Einfluss der künstlich erhöhten Gesamtphosphorkonzentration auf die planktische Primärproduktion im Verlaufe eines Jahres

Da Resultate aus Bioassay-Testen je nach der Wahl des Stichtags quantitativ unterschiedlich, nach STANGE-BURSCHE [76] sogar qualitativ entgegengesetzt ausfallen können, wurden zur generellen Beurteilung der Wirkung des zugesetzten Phosphors auf die Primärproduktion die Assimilationsraten aller Versuchstage arithmetisch gemittelt und die mittlere Assimilationsrate der Vergleichszelle $\left(\mathrm{NTZ}_{0}\right)$ sowie ihr mittlerer Gehalt an Gesamtphosphor gleich 1 gesetzt.

In Abb. 17 ist die relative Erhöhung des Gesamtphosphors und die dadurch bewirkte relative Veränderung der Assimilationsraten graphisch dargestellt. Es ist daraus ersichtlich, dass im Winter eine Phosphordüngung keine Steigerung der Primärproduktion bewirkt. Es muss angenommen werden, dass zu dieser Zeit das Ausmass der Primärproduktion bei der gegebenen Nährstoffkonzentration durch die niedrige Temperatur und die geringe Einstrahlung begrenzt wird.

$\square$

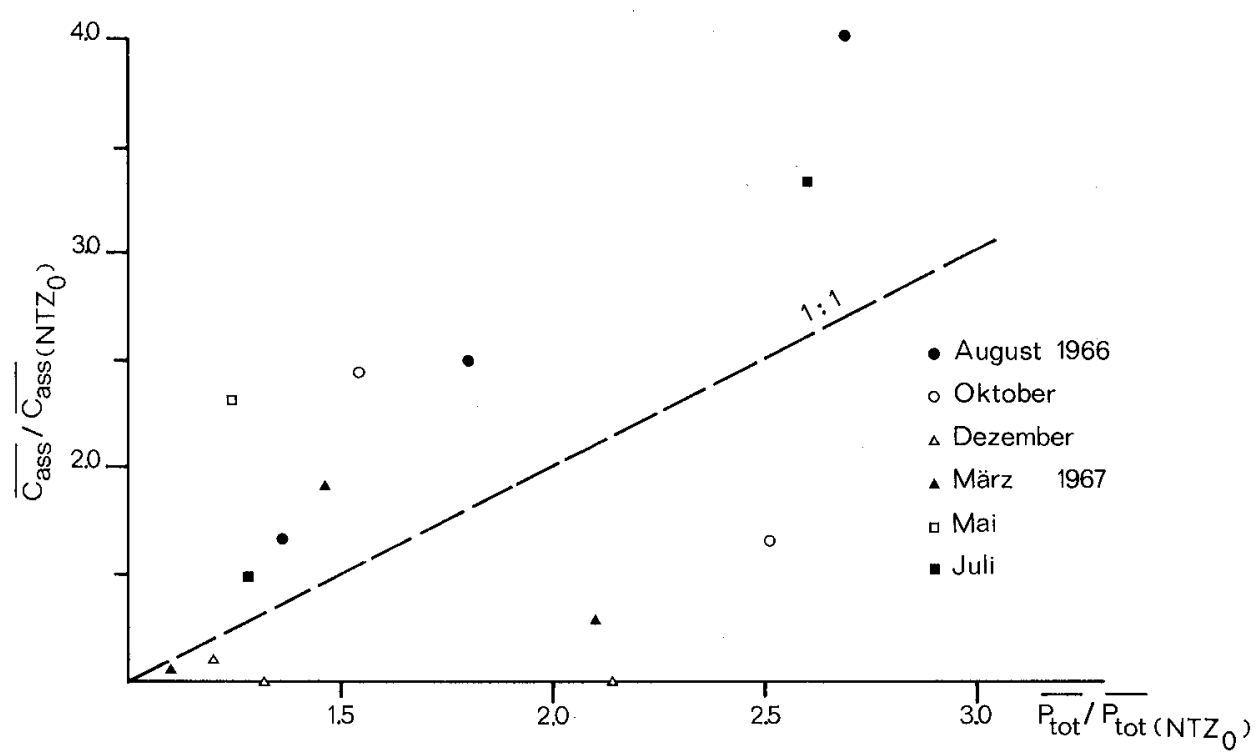

Abb. 17. Relative Veränderung der mittleren Gesamtphosphorkonzentration und die dadurch bewirkte relative Veränderung der mittleren Kohlenstoffassimilationsraten. $C_{\text {ass }}$ und $P_{\text {tot }}$ der ungedüngten Kontrolle $\left(\mathrm{NTZ}_{\mathbf{0}}\right)$ wurden gleich 1 gesetzt.

Im März wurde mit einer Erhöhung der Gesamtphosphorkonzentration um 50\% beinahe eine Verdoppelung der Assimilationsrate erreicht. Bei einer weiteren Steigerung des Phosphorgehalts auf $210 \%$ des natürlichen Wertes fiel die mittlere Assimilationsrate wieder wesentlich kleiner aus und übertraf den Kontrollwert nur noch um $27 \%$. Diese Erscheinung kann nur damit erklärt werden, dass mit der künstlichen Erhöhung der Phosphorkonzentration in der $\mathrm{NTZ}_{30}$ die für die damaligen Verhältnisse optimale Konzentration dieses Nährstoffs bereits überschritten war. 
Im Mai zeigte die Phosphatdüngung maximale Wirkung, obwohl die natürliche Gesamtphosphorkonzentration, bedingt durch eine verstärkte Phosphorzufuhr zur trophogenen Schicht, wesentlich höher lag als im Dezember und März. Das zeigt, dass weder Konzentrationsmessungen noch die Ermittlung der Nährstoffzufuhr über die begrenzende Wirkung eines Nährstoffs etwas aussagen.

Die Versuche vom Juli/August 1966 und Juli 1967 ergaben beinahe dasselbe Resultat, nämlich einen angenähert linearen Zusammenhang zwischen der relativen Erhöhung der Gesamtphosphorkonzentration und derjenigen der Kohlenstoffassimilation. Das Verhältnis $\Delta \mathrm{C}: \Delta \mathrm{P}$ fiel jedoch wesentlich kleiner aus als im Mai. Dies mag, von der andern qualitativen Zusammensetzung des Planktons abgesehen, auch damit zusammenhängen, dass zu dieser Zeit bereits auch andere Nährstoffe so weit aufgezehrt sind, dass die Differenz zwischen den mit und ohne Phosphordüngung erreichbaren Erträgen kleiner geworden ist als im Frühjahr.

Das Resultat des Düngeversuchs im Oktober deckt sich im wesentlichen mit demjenigen vom März. Eine Erhöhung der Gesamtphosphorkonzentration um $54 \%$ bewirkte eine Steigerung der Photosyntheserate um 144\%. Wurde die Phosphorkonzentration noch bis auf den 2,5fachen Wert der Kontrolle erhöht, so nahm die Primärproduktion wieder merklich ab.

Dass eine Verdoppelung des Phosphorgehalts eine Steigerung der Assimilationsraten um mehr als $100 \%$ bewirken kann, steht, so formuliert, im Widerspruch zum Gesetz von MitscherLICH [3], nach welchem eine Verdoppelung der Nährstoffkonzentration höchstens eine Verdoppelung des Ertrags bewirken kann. Dieser angenähert lineare Zusammenhang besteht zudem nur bei den sehr niedrigen Konzentrationen des untersten suboptimalen Bereichs. Die Diskrepanz zwischen unsern Beobachtungen und dem Mitscherlichschen Gesetz ist zur Hauptsache damit zu erklären, dass in natürlichem Seewasser derjenige Teil des Gesamtphosphors, der momentan photosynthetisch wirksam ist, durch die Düngung mit leicht "assimilierbarem» Phosphat um ein vielfaches stärker vermehrt wird als die Gesamtphosphorkonzentration. Obwohldaher die Gesamtphosphorkonzentration für physiologische Erwägungen wenig Aussagekraft hat, bleibt sie, da sich zugeführtes Phosphat durch rasche Inkorporation im Phytoplankton der chemischen Analyse entzieht, für den Limnologen doch eine brauchbare Grösse, die ihn zuverlässig über die Phosphorversorgung eines Gewässers orientiert.

Quantitativ können die im Experiment gewonnenen Resultate nicht auf die natürlichen Verhältnisse im See übertragen werden. Immerhin kann daraus aber allgemein gefolgert werden, dass während der wichtigsten Vegetationszeit die Wirkung aller andern Faktoren genügt, um bei gesteigerter Phosphatzufuhr eine erhöhte Produktion zu ermöglichen. Der oft angenähert lineare Zusammenhang zwischen der Änderung der Phosphatkonzentration und der Änderung des Ertrags im Experiment deutet darauf hin, dass die natürliche Phosphorkonzentration für das Phytoplankton im gesamten gesehen stark suboptimal ist. Daraus folgt, dass das untersuchte Gewässer auf eine Veränderung im Phosphorhaushalt äusserst empfindlich reagieren würde. Wenn daher durch Sanierungsmassnahmen die laufende Phosphorzufuhr wirksam verringert werden könnte, so müsste sich das zwangsläufig in einer wesentlich niedrigeren Primärproduktion auswirken. Dass sich nach dem Fernhalten von Abwässern im See tatsächlich rasch Erfolge dieser Sanierungsmassnahme einstellen können, wurde von LiEPolt [77] am Beispiel des Zellersees gezeigt. 


\section{Schlussfolgerungen}

Monatliche Erhebungen und direkte Messungen der Sedimentationsraten haben gezeigt, dass im untersuchten Gewässer der trophogenen Schicht auch während der Stagnationsperiode dauernd Phosphorverbindungen zugeführt werden (s. S. 22 ff.).

Aus direkten Messungen der Primärproduktion im See geht hervor, dass die wichtigste Vegetationszeit mit der Sommerstagnation zusammenfällt.

Es wurde experimentell nachgewiesen, dass vom Frühling bis zum Herbst ein sehr enger Zusammenhang zwischen der Primärproduktion und der Gesamtphosphorkonzentration besteht (Abb. 17).

Die Gesamtphosphorkonzentration der trophogenen Schicht ist während der Stagnationsperiode ihrerseits abhängig von der Basisphosphorkonzentration des Gewässers während der Frühjahrszirkulation, von den täglichen Sedimentationsverlusten und der täglichen Phosphorzufuhr. Es ist daher zu erwarten, dass in jenen Fällen, in welchen eine begrenzende Wirkung des Phosphors nachgewiesen ist und in welchen die tägliche Phosphorzufuhr zur trophogenen Schicht durch technische Sanierungsmassnahmen (Ringleitung oder Phosphorelimination in der dritten Reinigungsstufe) entscheidend gedrosselt werden kann, die Primärproduktion als Sofortwirkung zumindest in der zweiten Hälfte der Stagnationsperiode kleiner werden muss, vorausgesetzt, dass sich die qualitative Zusammensetzung des Phytoplanktons nicht entscheidend verändert. Wie rasch eine Verdünnung der eingeleiteten Nährstoffe gegen die Seemitte hin erfolgt und dadurch diese sofortige Wirkung örtlich begrenzt wird, kann im Moment allerdings nicht beurteilt werden.

Wie rasch und wie weit sich die getroffenen Sanierungsmassnahmen auch auf die Basisphosphorkonzentration auswirken, hängt wesentlich davon ab, wie schnell sich das Wasser eines Sees erneuert, wie stark und wie lange er thermisch geschichtet ist und wie gross die unkontrollierbare Restbelastung (im Vergleich zum jährlichen Verlust durch Abfluss und irreversible Fixierung im Sediment) bleibt. Da diese Fixierung bei sonst gleichen Bedingungen unter aeroben Verhältnissen wesentlich grösser ist, als unter Anaerobiose [79] sind die Erfolgsaussichten für die Sanierungsmassnahmen um so grösser, je früher sie getroffen werden. Vom limnologischen Standpunkt aus muss aber ein Fernhalten von Phosphaten von stehenden Gewässern auch dann gefordert werden, wenn sich trotz aller technisch möglichen Sanierungsmassnahmen nur ein Teil der Zufuhr erfassen und damit nur die Akkumulierungsgeschwindigkeit der Basisphosphorkonzentration verringern lässt. Dadurch würde die seit Jahren beobachtete zunehmende Eutrophierung zwar nicht rückgängig gemacht, könnte aber in ihrer Entwicklung doch gestoppt oder zumindest verlangsamt werden.

\section{Zusammenfassung}

Die Horwer Bucht, ein Becken des Vierwaldstättersees, wurde während 2 Jahren untersucht. Durch die chemische Analyse wurden die folgenden Komponenten erfasst: Sauerstoff, Nitrat-Stickstoff, Phosphat-Phosphor $\left(\mathrm{PO}_{4}\right)$, gelöste "organische» Phosphorverbindungen (GOP) und partikuläre Phosphorverbindungen (PP) sowie SBV und $\mathrm{pH}$-Wert. Die wichtigsten Formen des Phytoplanktons wurden im umgekehrten Mikroskop ausgezählt und die Primärproduktion während eines Jahres 
monatlich mit der $\mathrm{C}^{14}$-Methode in situ bestimmt. Gleichzeitig wurde die Temperatur und die subaquatische Veränderung des Lichtes gemessen.

Aufgrund der Nährstoffkonzentration während der Vollzirkulation (15-20 $\mu \mathrm{g}$ Gesamt-P/1 und rund $350 \mathrm{mg} \mathrm{NO}_{3}-\mathrm{N} / \mathrm{l}$ ) sowie aufgrund der vertikalen Verteilung der Sauerstoffkonzentrationen während der Sommerstagnation handelt es sich um ein mesotrophes Gewässer. Das Auftreten von deutlichen Produktionsmaxima in der vertikalen Verteilung der planktischen Primärproduktion sowie die Tatsache, dass Oscillatoria rubescens und Tabellaria fenestrata während des ganzen Jahres die wichtigsten Vertreter des Phytoplanktons waren, deuten ebenfalls auf einen fortgeschrittenen Eutrophierungszustand hin.

Direkte Messungen haben ergeben, dass täglich rund 2\% des PP der trophogenen Schicht durch Sedimentation verlorengehen. Da sich diese Sedimentationsverluste aber nicht in einer ihnen entsprechenden Abnahme der Gesamtphosphorkonzentration bemerkbar machen, muss angenommen werden, dass der trophogenen Schicht auch während der Stagnationsperiode ständig Phosphor zugeführt wird. Als mögliche Quellen werden das Hypolimnion und das pelagische Sediment, das Sediment und die Flora der Litoralzone sowie die tägliche Abwasserzufuhr diskutiert. Es zeigte sich, dass im Falle der Horwer Bucht das Abwasser wahrscheinlich als wesentlichste Phosphorquelle zu betrachten ist. Aufgrund der Kohlenstoffassimilationsraten wird versucht, die täglich umgesetzte Menge Phosphor abzuschätzen. Es zeigt sich, dass die tägliche Zufuhr etwa $30 \%$ der täglich umgesetzten Menge ausmacht.

In speziell konstruierten Plastikbehältern von etwa 14001 Kulturvolumen (Abb. 10) wurde die Wirkung von Phosphatdüngungen auf das Phytoplankton in situ geprüft. Das Phosphat wurde in Form von $\mathrm{NaH}_{2} \mathrm{PO}_{4} \cdot 2 \mathrm{H}_{2} \mathrm{O}$ (Merck) zum Seewasser zugegeben. Die natürliche Gesamtphosphorkonzentration wurde dadurch, von einem Extremfall abgesehen, höchstens verdreifacht. Die zeitliche Veränderung der Konzentrationen der drei Phosphorkomponenten $\mathrm{PO}_{4}$, GOP und PP wurde verfolgt (Abb. 11-16), die Primärproduktion unter konstanten Bedingungen im Rotationslichtthermostaten gemessen (Tab. 16, 18, 20, 22, 24, 26) und Veränderungen im Bestand des Phytoplanktons durch Auszählen von Stichproben im umgekehrten Mikroskop festgehalten (Tab. 17, 19, 21, 23, 25, 27, 28).

Das zugesetzte Phosphat $\left(\mathrm{PO}_{4}\right)$ wurde vom Frühahr bis zum Herbst rasch in partikulären Phosphor (PP) übergeführt. Im Winter konnte dieser Vorgang nicht beobachtet werden. Die Sedimentationsraten von PP waren in der Regel um so grösser, je rascher das zugesetzte Phosphat aufgenommen wurde und je höher bei Versuchsbeginn die Anfangskonzentration war. Vom März bis zum Oktober konnte die planktische Primärproduktion durch die Phosphatdüngung eindeutig gesteigert werden (Tab. 16, 18, 22, 24, 26; Abb. 17).

Die verschiedenen Algenspezies reagierten auf die Phosphatdüngung unterschiedlich. Es zeigte sich, dass eine Steigerung der Phosphatkonzentration genügte, um das Wachstum der Chlorophyceen und Diatomeen zu stimulieren. Die Chrysophyceen (Dinobryon spp.) wurden durch die Phosphatzugabe in der Zellteilung gehemmt, und Oscillatoria rubescens reagierte auf die Phosphatdüngung verglichen mit den Grünalgen kaum (Tab. 28).

Aus diesen Beobachtungen wird abgeleitet, dass eine Unterbindung der Phosphorzufuhr zur trophogenen Schicht sich während der Stagnationsperiode, gleichbleibende 
Biozönose vorausgesetzt, rasch in einer kleiner werdenden Primärproduktion bemerkbar machen muss. Ob und wieweit sich durch Sanierungsmassnahmen auch die Basisphosphorkonzentration verringern lässt, hängt von verschiedenen Faktoren, darunter wesentlich von den hypolimnischen Sauerstoffverhältnissen ab. Die Erfolgsaussichten sind deshalb um so grösser, je rascher die Sanierungsmassnahmen getroffen werden.

\section{SUMMARY}

The Horw Bay (Lake of Lucerne) was investigated over a period of two years. The following components were investigated: oxygen, nitrate-nitrogen, phosphate-phosphorus $\left(\mathrm{PO}_{4}\right)$, dissolved 'organic' phosphorus compounds (DOP) and particular phosphorus compounds (PP); moreover, alkalinity and $\mathrm{pH}$-value were determined. The main forms of phytoplankton were counted by means of an inverted microscope and the primary production was determined monthly over a period of one year (C-14 method). At the same time, the temperature and subsurface light conditions were measured.

Considering the nutrient concentration during circulation (15-20 $\mu \mathrm{g}$ total-P/I and approximately $350 \mathrm{mg} \mathrm{NO}-\mathrm{N} / 1)$ and the vertical distribution of oxygen concentrations during summer stagnation, this portion of the lake may be characterized as mesotrophic. The distinct production maxima in the vertical distribution of primary planktic production, as well as the fact that, throughout the year, Oscillatoria rubescens and Tabellaria fenestrata were the main constituents of the phytoplankton, also indicate that this portion of the lake is in an advanced state of eutrophication.

Direct measurements have shown that approximately 2\% PP from the trophogenic layer are lost daily by sedimentation. However since these sedimentation losses are not followed by a corresponding decrease of the total phosphorus concentration, it must be supposed that the trophogenic layer receives constant supplies of phosphorus even during the stagnation period.

The possible sources of phosphorus could be the hypolimnion and the pelagic sediment, the sediment and the flora of the littoral zone, as well as the daily introduction of sewage. In the case of the Horw Bay, sewage is probably the chief supplier of phosphorus.

The rates of carbon assimilation were taken as a basis for evaluating the amount of phosphorus which is transformed daily. The daily supply amounts to about $30 \%$ of the quantity which is transformed per day.

The fertilizing influence of phosphates on the phytoplankton was tested in situ in especially constructed plastic containers with a capacity of about 1400 litres of culture (Fig. 10). Phosphate $\left(\mathrm{NaH}_{2} \mathrm{PO}_{4} \cdot 2 \mathrm{H}_{2} \mathrm{O}\right.$ p.a. Merck) was added to the lake water in an amount such that natural total phosphorus concentration was, at most, tripled, except for one extreme case. Observations were made on the temporal modifications of the concentrations of the three phosphorus components $\mathrm{PO}_{4}, \mathrm{DOP}$ and PP (Fig. 11-16). The primary production under constant conditions was measured in the rotational light thermostat (Tables 16, 18, 20, 22, 24, 26). To monitor the phytoplankton composition, samples were examined and counted by means of an inverted microscope (Tables 17, 19, 21, 23, 25, 27, 28).

From spring to autumn, the phosphate $\left(\mathrm{PO}_{4}\right)$ added to the containers was rapidly transformed into particular phosphorus (PP). This change, however, was not observed in winter. Generally, the more rapid the phosphate uptake and the higher the initial concentration at the beginning of the experiment, the higher the sedimentation rates of PP.

The results of this investigation show that from March to October, the planktic primary production could clearly be increased by phosphate fertilization (Tables 16, 18, 22, 24, 26, and Fig. 17).

The various species of algae, however, reacted differently to the phosphate fertilization. An increase of the phosphate concentration was sufficient to stimulate the growth of Chlorophyceae and Diatomeae. In contrast, the cell proliferation of Chrysophyceae (Dinobryon spp.) was retarded by the phosphate fertilization. As compared with green algae, Oscillatoria rubescens hardly reacted to the phosphate fertilization (Table 28). 
The following deductions may be drawn from the above observations: During the stagnation period, any decrease of the phosphorus supply to the trophogenic layer must result in a rapid decrease of the primary production, provided there is no change in the biocenose. The question as to whether and to what extent it would also be possible to reduce the residual phosphorus concentration by applying meliorational measures depends on scveral factors and especially on the hypolimnic oxygen conditions. The sooner the remedial measures are taken, the greater will be the chances of success.

\section{LITERATURVERZEICHNIS}

[1] E. A. Thomas, Verh. int. Verein. theor. angew. Limnol. 10, 489-495 (1949).

[2] H. ZÜLLIG, Schweiz. Z. Hydrol. 18, 5-143 (1956).

[3] L. Mitscherlich, Schr. königsb. gelehrt. Ges. naturw. Kl. 3, 1-29 (1927).

[4] J. Verduin, Science 175, 23 (1952).

[5] D. Schwarz, Der Einfluss von Wirkstoffen auf das Wachstum und die Vermehrung von Algen, Veröffentlichungen der Hydrologischen Forschungsabteilung der Dortmunder Stadtwerke AG, Nr. 8, 206 S. (1965).

[6] F. E. Fritsch, P. K. DE, Nature 142, 878 (1938).

[7] V. A. Dugdale, R. C. Dugdale, Limnol. Oceanogr. 10, 53-57 (1965).

[8] W. Einsele, Z. Fisch. 39, 407-488 (1941).

[9] G. E. Hutchinson, Ecol. Monogr. 11, 23-60 (1941).

[10] C. N. SAwYER, Sewage ind. Wastes Engng. 24, 768-776 (1952).

[11] E. A. Thomas, Schweiz. Ver. von Gas- und Wasserfachmännern, Mbull. 33, Nr, 2 und 3, 1-15 (1953).

[12] H. АмвӥнL, Schweiz. Z. Hydrol. 26, 569-594 (1964).

[13] J. W. G. LUND, Biol. Rev. 40, 231-293 (1965).

[14] F. J. Mackereth, J. exp. Bot. 4, 296-313 (1953).

[15] F. GEssne, Hydrobotanik $I T$, S. 540 (VEB Deutscher Verlag der Wissenschaften, Berlin 1959).

[16] R. KLiffü̈Lle R, Int. Revue ges. Hydrobiol. Hydrogr. 47, 118-122 (1962).

[17] J. GRIM, GWF 108, 1261-1271 (1967).

[18] G. Bringmann, R. KÜHN, Gesundheitsingenieur 84 (12), 364-365 (1963).

[19] O. M. Skulberg, Advances in Water Pollution Research, Proc. 3rd Int. Conf. Wat. Pollut. Res. Munich 1, 113-129 (1966).

[20] C. R. Goldman, Science 132, 1016-1017 (1960).

[21] J. H. Ryther, R. R. L. Guillard, Deep Sea Res. 6 (1), 65-69 (1959).

[22] D. W. Menzel, J. H. Ryther, Deep Sea Res. 7 (4), 276-281 (1961).

[23] C. R. Goldman, Limnol. Oceanogr. 7, 99-101 (1962).

[24] J. D. H. STRICKLAND, Limnol. Oceanogr. 8, 166-183 (1963).

[25] J. D. H. StrickLand, Limnol. Oceanogr. 6, 93-96 (1961).

[26] L. W. Winkler, Chem. Ber. 21, 2843-2855 (1888).

[27] H. UtermöhL, Int. Verein, theor. und angew. Limnol. Mitt. Nr. 9, 38 S. (1958).

[28] H. AmвüHL, Schweiz. Z. Hydrol. 22, 23-39 (1960).

[29] H. W. Harvex, W. RodHe, The Chemistry and Fertility of Sea Waters (Cambridge University Press, London 1955).

[30] W. OHLE, Z. anorg. Chem. 49, 206 (1936).

[31] H. АмвӥнL, M. Schмid, Schweiz. Z. Hydrol. 27, 172-183 (1965).

[32] M. Sснмid, H. Aмвüнl, Schweiz. Z. Hydrol. 27, 184-192 (1965).

[33] R. Müller, O. WidemanN, Jahrbuch v. Wasser, 22 247-271 (1955).

[34] Deutsche Einheitsverfahren zur Wasseruntersuchung, 3. Aufl. (1960).

[35] E. Steeman-Nielsen, J. Cons. 18, 117-140 (1952).

[36] J. SchwöRBEL, Methoden der Hydrobiologie, S. 207 (Francksche Verlagshandiung, W. Keller \& Co., Stuttgart 1966).

[37] A. ThIEnEmann, Der Sauerstoff im eutrophen und oligotrophen See, Die Binnengewässer 4 (Schweizerbartsche Verlagsbuchhandlung, Stuttgart 1928).

[38] H. Schmassmann, Schweiz. Z. Hydrol. 18, 144-155 (1956). 
[39] B. Åberg, W. Rodhe, Über die Mitieufaktoren in einigen siidschwedischen Seen, Symb. bot. upsal. 5 (3) (1942).

[40] R. VolL.enweIder, Die wissenschaftlichen Grundlagen der Seen- und Fliessgewässereutrophierung, unter besonderer Berücksichtigung des Phosphors und des Stickstoffs als Eutrophierungsfaktoren (Manuskript).

[41] F. H. RIGLER, Verh. int. Verein. theor. angew. Limnol. 16, 465-470 (1966).

[42] J. D. H. Strickland, T. R. Parsons, Bull. Fish. Res. Bd. Can. 125, 1-185 (1960).

[43] F. H. Rigler, Limnol. Oceanogr. 9, 517-518 (1964).

[44] S. P. CHU, J. mar. biol. Ass. 26, 285-295 (1946).

[45] W. RodHe, Symb. bot. upsal. 10 (1) (1948).

[46] L. Provasoli, A. Rev. Microbiol. 12, 279-308 (1958).

[47] R. A. Galloway, R. W. Krauss, Microalgae and Photosynthetic Bacteria (Japanese society of plant physiologists, The Univ. of Tokyo Press 1963).

[48] J. OvERBECK, Arch. Hydrobiol. 58, 281-308 (1962).

[49] C. ForsberG, D. Jinnerot, L. Davidsson, Vatten 23 (1) 2-16 (1967).

[50] J. OverbeCK, Arch. Hydrobiol. 58, 162-209 (1962).

[51] W. D. Watt, F. R. Hayes, Limnol. Oceanogr. 8, 276-285 (1963).

[52] J. P. Barlow, Limnol. Oceanogr. 10 Suppl. R15-R24 (1965).

[53] R. E. Johannes, Sience 146, 923-924 (1964).

[54] R. E. Johannes, Limnol. Oceanogr. 10, 434-442 (1965).

[55] L. R. Pomeroy, H. M. Mathews, H. S. Min, Limnol. Oceanogr. 8, 50-55 (1963).

[56] F. H. Rigler, Limnol. Oceanogr. 6, 165-174 (1961).

[57] F. H. Rigler, Can. Fish Cult. 32, 3-9 (1964).

[58] E. A. Thomas, Schweiz. Ver. von Gas- und Wasserfachmännern, Mbull. 41, Nr. 3, 4, 3-16 (1961).

[59] K. Wuhrmann, Föderation Europäischer Gewässerschutz (FEG), Informationsblatt Nr. 14, 67-73 (1967).

[60] H. АмвÜHL, GWF 107, 357-363 (1966).

[61] P. Javornicky, Sci. Pap. Inst. chem. Techn., Prague, Fac. Techn. Fuel and Water 2, 283-367 (1958).

[62] H. Lohmann, Wiss. Meeresunters. 10, 181. (1908).

[63] A. C. Redfield, B. H. Ketchum, F. A. Richards The Sea 2 (Wiley and Sons, New YorkLondon 1963).

[64] H. L. Goltermann, Acta bot. neerl. 9, 1-58 (1960).

[65] W. Rodhe, Memorie Ist. ital. Idrobiol. 18 Suppl. 365-381 (1965).

[66] U. ZImMERManN (Dissertation ETH, noch in Arbeit).

[67] A. NAuWERCK, Symb. bot. upsal. 77 (5), 1-163 (1963).

[68] B. H. Кетснuм, Am. J. Bot. 26, 399-407 (1939).

[69] V. D. Ramamurthy, S. Krishnamurthy, Proc. Indian Acad. Sci. [B] 65, 43-48 (1967).

[70] I. FindEnEGG, Föderation Europäischer Gewässerschutz (FEG), Informationsblatt Nr. 14, 11-17 (1967).

[71] R. Staub, Schweiz. Z. Hydrol. 23, 82-198 (1961).

[72] G. C. Gerloff, F. SKoog, Ecology 35, 348-353 (1954).

[73] M. Pavoni, Schweiz. Z. Hydrol. 25, 219-336 (1963).

[74] M. Pavoni (in Vorbereitung).

[75] R. A. Vollenweider, Schweiz. Z. Hydrol. 12, 193-262 (1950).

[76] E. M. Stange-Bursche, Int. Revue ges. Hydrobiol. Hydrogr. 49, 361-374 (1964).

[77] R. LIEPOLr, Föderation Europäischer Gewässerschutz (FEG), Informationsblatt Nr. 14, 59-62 (1967).

[78] J. OVERBECK, Ber. dt. bot. Ges. 76, 276-286 (1963).

[79) E. A. Thomas, Memorie Ist. ital. Idrobiol. 8 Suppl. 357-465 (1955).

Separatdruck aus der Schweiz. Zeitschrift für Hydrologie 30, Fasc. 1 (1968) Herausgegeben mit Unterstützung der Stiftung der Wirtschaft zur Förderung des Gewässerschutzes in der Schweiz 